\title{
Tutte polynomial, subgraphs, orientations and sandpile model: new connections via embeddings
}

\author{
Olivier Bernardi* \\ CNRS, Université Paris-Sud, Bât 425, 91405 Orsay Cedex, France \\ olivier.bernardi@math.u-psud.fr \\ Submitted: Jan 23, 2007; Accepted: Aug 13, 2008; Published: Aug 25, 2008 \\ Mathematics Subject Classification: 05C20
}

\begin{abstract}
We define a bijection between spanning subgraphs and orientations of graphs and explore its enumerative consequences regarding the Tutte polynomial. We obtain unifying bijective proofs for all the evaluations $T_{G}(i, j), 0 \leq i, j \leq 2$ of the Tutte polynomial in terms of subgraphs, orientations, outdegree sequences and sandpile configurations. For instance, for any graph $G$, we obtain a bijection between connected subgraphs (counted by $T_{G}(1,2)$ ) and root-connected orientations, a bijection between forests (counted by $T_{G}(2,1)$ ) and outdegree sequences and bijections between spanning trees (counted by $T_{G}(1,1)$ ), root-connected outdegree sequences and recurrent sandpile configurations.

All our proofs are based on a single bijection $\Phi$ between the spanning subgraphs and the orientations that we specialize in various ways. The bijection $\Phi$ is closely related to a recent characterization of the Tutte polynomial relying on combinatorial embeddings of graphs, that is, on a choice of cyclic order of the edges around each vertex.
\end{abstract}

\section{Introduction}

In 1947, Tutte defined a graph invariant that he named the dichromate because he thought of it as bivariate generalization of the chromatic polynomial [42]. Since then, the dichromate, now known as the Tutte polynomial, has been widely studied (see $[5,7])$.

*This work was partially supported by the Centre de Recerca Matemàtica of Barcelona and by the French Agence Nationale de la Recherche, project SADA ANR-05-BLAN-0372. 
There are several alternative definitions of the Tutte polynomial [3, 23, 32, 43]. The most straightforward definition for a connected graph $G=(V, E)$ is

$$
T_{G}(x, y)=\sum_{S \text { spanning subgraph }}(x-1)^{c(S)-1}(y-1)^{c(S)+|S|-|V|},
$$

where the sum is over all spanning subgraphs $S$ (equivalently, subsets of edges), $c(S)$ denotes the number of connected components of $S$ and |.| denotes cardinality. From this definition, it is easy to see that $T_{G}(1,1)$ (resp. $T_{G}(2,1), T_{G}(1,2)$ ) counts the spanning trees (resp. forests, connected subgraphs) of $G$. More surprisingly, all the specializations $T_{G}(i, j), 0 \leq i, j \leq 2$ as well as some of their refinements have nice interpretations either in terms of orientations $[24,28,32,33,40]$ outdegree sequences [7, 41] or sandpile configurations $[10,34]$.

A number of articles have been devoted to combinatorial proofs of the specializations of the Tutte polynomial $[21,22,23,24,25,33]$. In this paper, we give unifying bijective proofs for the interpretation of each of the evaluations $T_{G}(i, j), 0 \leq i, j \leq 2$ in terms of orientations and outdegree sequences. The strength of our approach is to derive all these interpretations from a single bijection $\Phi$ between subgraphs and orientations that we specialize in various ways. Indeed, for any graph $G$, the mapping $\Phi$ induces a bijection between:

- root-connected orientations and connected subgraphs (counted by $T_{G}(1,2)$ ),

- minimal orientations (which are in bijection with outdegree sequences) and forests (counted by $T_{G}(2,1)$ ),

- strongly connected orientations and external subgraphs (counted by $T_{G}(0,2)$ ),

- acyclic orientations and internal forests (counted by $T_{G}(2,0)$ ),

- root-connected minimal orientations (which are in bijection with root-connected outdegree sequences) and spanning trees (counted by $T_{G}(1,1)$ ),

- strongly connected minimal orientations (which are in bijection with strongly-connected outdegree sequences) and external spanning trees (counted by $T_{G}(0,1)$ ),

- root-connected acyclic orientations and internal spanning trees (counted by $T_{G}(1,0)$ ).

The enumerative corollaries of these bijections are not new. The enumeration of acyclic orientations by $T_{G}(2,0)$ was first established by Winder in 1966 [45] and rediscovered by Stanley 1973 [40]. The result of Winder was stated as an enumeration formula for the number of faces of hyperplanes arrangements and was independently extended to real arrangements by Zaslavsky [46] and to orientable matroids by Las Vergnas [31]. The enumeration of root-connected acyclic orientations by $T_{G}(1,0)$ was found by Greene and Zaslavsky [28]. In [23], Gessel and Sagan gave a bijective proof of both results. In [21], Gebhard and Sagan gave three other proofs of Greene and Zaslavsky's result. The enumeration of strongly connected orientations by $T_{G}(0,2)$ is a direct consequence of Las Vergnas' characterization of the Tutte polynomial [32]. The enumeration of outdegree sequences by $T_{G}(2,1)$ was discovered by Stanley [41] and a bijective proof was established in [29]. The enumeration of root-connected orientations by $T_{G}(1,2)$, the enumeration of root-connected outdegree sequences by $T_{G}(1,1)$ and the enumeration of strongly con- 
nected outdegree sequences by $T_{G}(0,1)$ were proved by Gioan in [24].

We shall also consider some specializations of the bijection $\Phi$ to some refined classes of orientations (such as bipolar orientations) considered in [25, 28, 33].

We shall also deal with the sandpile model $[1,18]$ (equivalently chip firing game [4]). It is known that the recurrent configurations of the sandpile model on $G$ (equivalently $G$-parking functions [39]) are counted by $T_{G}(1,1)[18]$. Observe that this is the number of spanning trees. The following refinement is also true: the coefficient of $y^{k}$ in $T_{G}(1, y)$ is the number of recurrent configurations at level $k$ [34]. A bijective proof of this result was given in [10]. We give an alternative bijective proof. We also answer a question of Gioan [24] by establishing a bijection between recurrent configurations of the sandpile model and root-connected outdegree sequences that leaves the configurations at level 0 unchanged.

Our bijections require a choice of a combinatorial embedding of the graph $G$, that is, a choice of a cyclic ordering of the edges around each vertex. In [3] the internal and external embedding-activities of spanning trees were defined for embedded graphs. It was proved that for any embedding of the graph $G$, the Tutte polynomial of $G$ is given by

$$
T_{G}(x, y)=\sum_{T \text { spanning tree }} x^{\mathcal{I}(T)} y^{\mathcal{E}(T)}
$$

where the sum is over all spanning trees $T$ and $\mathcal{I}(T)$ (resp. $\mathcal{E}(T)$ ) denotes the internal (resp. external) embedding-activity. This characterization of the Tutte polynomial is reminiscent but inequivalent to the one given by Tutte in [43]. The characterization (2) is our main tool in order to obtain enumerative corollary from our bijections. In this respect, our approach is close to the one used by Gessel and Sagan in [22, 23] in order to obtain enumerative consequences from a new notion of external activity.

The outline of this paper is as follows.

- In Section 2, we recall some definitions and preliminary results about graphs, orientations and the sandpile model.

- In Section 3, we take a glimpse at the results to be developed in the following sections. We first establish some elementary results about the tour of spanning trees and their embedding-activities. Then we define a mapping $\Phi$ from spanning trees to orientations. We highlight a connection between the embedding-activities of a spanning tree $T$ and the acyclicity or strong-connectivity of the orientation $\Phi(T)$. Building on the mapping $\Phi$ we also define a bijection $\Gamma$ between spanning trees to root-connected outdegree sequences and a closely related bijection $\Lambda$ between spanning trees and recurrent configurations of the sandpile model.

- In Section 4, we define a partition $\Pi$ of the set of subgraphs. Each part of this partition is an interval in the boolean lattice of the set of subgraphs and is associated to a spanning tree. The interval associated with a spanning tree $T$ is closely related to the embeddingactivities of $T$. Using results from [26], we show how the partition $\Pi$ explains the link 
between the subgraph expansion (1) and the spanning tree expansion (2) of the Tutte polynomial. We also consider several criteria for subgraphs: connected, forest, internal, external and prove that the families of subgraphs that can be defined by combining these criteria are counted by one of the evaluations $T_{G}(i, j), 0 \leq i, j \leq 2$ of the Tutte polynomial.

- In Section 5, we extend the mapping $\Phi$ to the set of all subgraphs. This definition makes use of the partition $\Pi$ of the set of subgraphs. We prove that $\Phi$ is a bijection between subgraphs and orientations.

- In Section 6, we study the specializations of the bijection $\Phi$ to the families of subgraphs defined by the criteria connected, forest, internal, external. We prove that $\Phi$ induces bijections between these families of subgraphs and the families of orientations defined by the criteria root-connected, minimal, acyclic, strongly connected. As a consequence, we obtain an interpretation for each of the evaluations $T_{G}(i, j), 0 \leq i, j \leq 2$ of the Tutte polynomial in terms of orientations or outdegree sequences.

- In Section 7, we study the bijection $\Lambda$ between spanning trees and recurrent configurations of the sandpile model.

- Lastly, in Section 8 we comment on the case of planar graphs.

\section{Definitions}

We denote by $\mathbb{N}$ the set of non-negative integers. For any set $S$, we denote by $|S|$ its cardinality. For any sets $S_{1}, S_{2}$, we denote by $S_{1} \triangle S_{2}$ the symmetric difference of $S_{1}$ and $S_{2}$. If $S \subseteq S^{\prime}$ and $S^{\prime}$ is clear from the context, we denote by $\bar{S}$ the complement of $S$, that is, $S^{\prime} \backslash S$. If $S \subseteq S^{\prime}$ and $s \in S^{\prime}$, we write $S+s$ and $S-s$ for $S \cup\{s\}$ and $S \backslash\{s\}$ respectively (whether $s$ belongs to $S$ or not).

\subsection{Graphs}

In this paper we consider finite, undirected graphs. Loops and multiple edges are allowed but, for simplicity, we shall only consider connected graphs. A spanning subgraph of a graph $G=(V, E)$ is a graph $G^{\prime}=\left(V, E^{\prime}\right)$ where $E^{\prime} \subseteq E$. All the subgraphs considered in this paper are spanning and we shall not further mention it. By convenience, we shall identify the subgraph with its edge set. A cut is a set of edges $C$ whose deletion increases the number of connected components and such that the endpoints of every edge in $C$ are in distinct components of the resulting graph. Given a subset of vertices $U$, the cut defined by $U$ is the set of edges with one endpoint in $U$ and one endpoint in $\bar{U}$. A cocycle is a cut which is minimal for inclusion (equivalently, it is a cut whose deletion increases the number of connected components by one). For instance, the set of edges $\{e, f, g, h, i, j\}$ in in Figure 1 and $\{f, g, h\}$ is a cocycle.

A forest is an acyclic graph. A tree is a connected forest. A spanning tree is a (spanning) subgraph which is a tree. Given a tree $T$ and a vertex distinguished as the root-vertex 


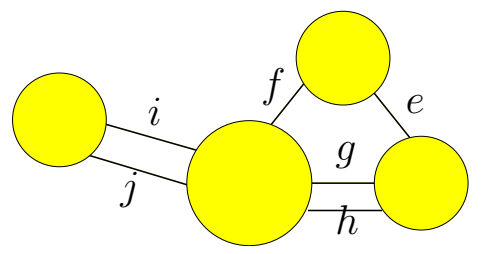

Figure 1: The cut $\{e, f, g, h, i, j\}$ and the connected components after deletion of this cut (shaded regions).

we shall use the usual family vocabulary and talk about the parent, child, ancestors and descendants of vertices in $T$. By convention, a vertex is considered to be an ancestor and a descendant of itself. If a vertex of the graph $G$ is distinguished as the root-vertex we implicitly consider it to be the root-vertex of every spanning tree.

Let $G$ be a graph and $T$ be a spanning tree. An edge of $G$ is said to be internal if it is in $T$ and external otherwise. The fundamental cycle (resp. cocycle) of an external (resp. internal) edge $e$ is the set of edges $e^{\prime}$ such that the subgraph $T-e^{\prime}+e\left(\operatorname{resp} . T-e+e^{\prime}\right)$ is a spanning tree. Observe that the fundamental cycle $C$ of an external edge $e$ is a cycle contained in $T+e$. Similarly, the fundamental cocycle $D$ of an internal edge $e$ is a cocycle contained in $\bar{T}+e$. Observe also that, if $e$ is internal and $e^{\prime}$ is external, then $e$ is in the fundamental cycle of $e^{\prime}$ if and only if $e^{\prime}$ is in the fundamental cocycle of $e$.

\subsection{Embeddings}

We recall the notion of combinatorial map [9, 11]. A combinatorial map (or map for short) $\mathcal{G}=(H, \sigma, \alpha)$ is a set of half-edges $H$, a permutation $\sigma$ and an involution without fixed point $\alpha$ on $H$ such that the group generated by $\sigma$ and $\alpha$ acts transitively on $H$. A map is rooted if one of the half-edges is distinguished as the root. For $h_{0} \in H$, we denote by $\mathcal{G}=\left(H, \sigma, \alpha, h_{0}\right)$ the map $(H, \sigma, \alpha)$ rooted on $h_{0}$. From now on all our maps are rooted.

Given a map $\mathcal{G}=\left(H, \sigma, \alpha, h_{0}\right)$, we consider the underlying graph $G=(V, E)$, where $V$ is the set of cycles of $\sigma, E$ is the set of cycles of $\alpha$ and the incidence relation is to have at least one common half-edge. We represent the underlying graph of the map $\mathcal{G}=(H, \sigma, \alpha)$ on the left of Figure 2, where the set of half-edges is $H=\left\{a, a^{\prime}, b, b^{\prime}, c, c^{\prime}, d, d^{\prime}, e, e^{\prime}, f, f^{\prime}\right\}$, the involution $\alpha$ is $\left(a, a^{\prime}\right)\left(b, b^{\prime}\right)\left(c, c^{\prime}\right)\left(d, d^{\prime}\right)\left(e, e^{\prime}\right)\left(f, f^{\prime}\right)$ in cyclic notation and the permutation $\sigma$ is $\left(a, f^{\prime}, b, d\right)\left(d^{\prime}\right)\left(a^{\prime}, e, f, c\right)\left(e^{\prime}, b^{\prime}, c^{\prime}\right)$. Graphically, we keep track of the cycles of $\sigma$ by drawing the half-edges of each cycle in counterclockwise order around the corresponding vertex. Hence, our drawing characterizes the map $\mathcal{G}$ since the order around vertices give the cycles of the permutation $\sigma$ and the edges give the cycles of the involution $\alpha$. On the right of Figure 2, we represent the map $\mathcal{G}^{\prime}=\left(H, \sigma^{\prime}, \alpha\right)$, where $\sigma^{\prime}=\left(a, f^{\prime}, b, d\right)\left(d^{\prime}\right)\left(a^{\prime}, e, c, f\right)\left(e^{\prime}, b^{\prime}, c^{\prime}\right)$. The maps $\mathcal{G}$ and $\mathcal{G}^{\prime}$ have isomorphic underlying graphs. 
Note that the underlying graph of a map $\mathcal{G}=(H, \sigma, \alpha)$ is always connected since $\sigma$ and $\alpha$ act transitively on $H$. A combinatorial embedding (or embedding for short) of a connected graph $G$ is a map $\mathcal{G}=(H, \sigma, \alpha)$ whose underlying graph is isomorphic to $G$ (together with an explicit bijection between the set $H$ and the set of half-edges of $G$ ). When an embedding $\mathcal{G}$ of $G$ is given we shall write the edges of $G$ as pairs of half-edges (writing for instance $e=\left\{h, h^{\prime}\right\}$ ). Moreover, we call root-vertex the vertex incident to the root and root-edge the edge containing the root. In the following, we use the terms combinatorial map and embedded graph interchangeably. We do not require our graphs to be planar.
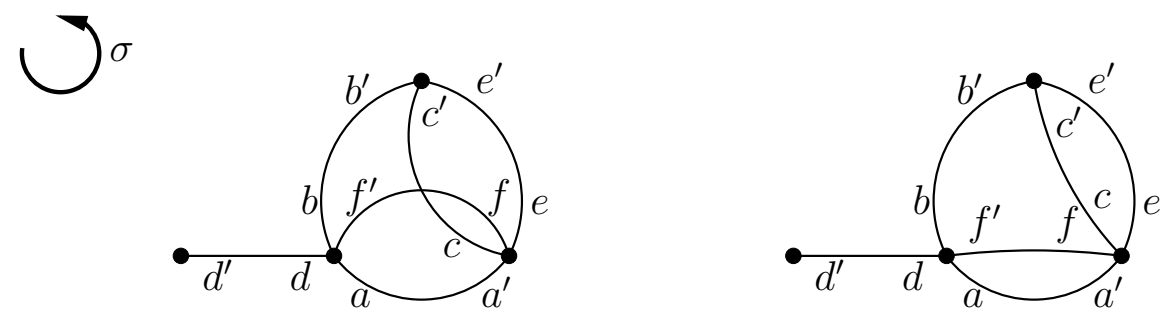

Figure 2: Two embeddings of the same graph.

Intuitively, a combinatorial embedding corresponds to the choice of a cyclic order on the edges around each vertex. This order can also be seen as a local planar embedding. In fact there is a one-to-one correspondence between combinatorial embeddings of graphs and the cellular embeddings of graphs in orientable surfaces (defined up to homeomorphism); see [36, Thm. 3.2.4].

\subsection{Orientations and outdegree sequences}

Let $G$ be a graph and let $\mathcal{G}$ be an embedding of $G$. An orientation is a choice of a direction for each edge of $G$, that is to say, a function $\mathcal{O}$ which associates to any edge $e=\left\{h_{1}, h_{2}\right\}$ one of the ordered pairs $\left(h_{1}, h_{2}\right)$ or $\left(h_{1}, h_{2}\right)$. Note that loops have two possible directions. We call $\mathcal{O}(e)$ an arc, or oriented edge. If $\mathcal{O}(e)=\left(h_{1}, h_{2}\right)$ we call $h_{1}$ the tail and $h_{2}$ the head. We call origin and end of $\mathcal{O}(e)$ the endpoint of the tail and head respectively. Graphically, we represent an arc by an arrow going from the origin to the end (see Figure 3).

$$
\text { origin } \underset{\text { tail head }}{\longrightarrow} \text { end }
$$

Figure 3: Half-edges and endpoints of arcs.

A directed path is a sequence of $\operatorname{arcs}\left(a_{1}, a_{2}, \ldots, a_{k}\right)$ such that the end of $a_{i}$ is the origin of $a_{i+1}$ for $1 \leq i \leq k-1$. A directed cycle is a simple directed closed path. A directed 
cocycle is a set of arcs $a_{1}, \ldots, a_{k}$ whose deletion disconnects the graph into two components and such that all arcs are directed toward the same component. If the orientation $\mathcal{O}$ is not clear from the context, we shall say that a path, cycle, or cocycle is $\mathcal{O}$-directed. An orientation is said to be acyclic (resp. totally cyclic or strongly connected) if there is no directed cycle (resp. cocycle).

We say that a vertex $v$ is reachable from a vertex $u$ if there is a directed path $\left(a_{1}, a_{2}, \ldots, a_{k}\right)$ such that the origin of $a_{1}$ is $u$ and the end of $a_{k}$ is $v$. If $v$ is reachable from $u$ in the orientation $\mathcal{O}$ denote it by $u_{\rightarrow}^{\mathcal{O}} v$. An orientation is said to be $u$-connected if every vertex is reachable from $u$. It is known that every edge in an oriented graph is either in a directed cycle but not both [35]. Hence, an orientation $\mathcal{O}$ is strongly connected if and only if the origin of every arc is reachable from its end. Equivalently, $\mathcal{O}$ is strongly connected if every pair of vertices are reachable from one another.

The outdegree sequence (or score vector) of an orientation $\mathcal{O}$ of the graph $G=(V, E)$ is the function $\delta: V \rightarrow \mathbb{N}$ which associates to every vertex the number of incident tails. We say that $\mathcal{O}$ is a $\delta$-orientation. The outdegree sequences are strongly related to the cycle flips, that is, the reversing of every edge in a directed cycle. Indeed, it is known that the outdegree sequences are in one-to-one correspondence with the equivalence classes of orientations up to cycle flips [20].

There are nice characterizations of the functions $\delta: V \rightarrow \mathbb{N}$ which are the outdegree sequence of an orientation. Given a function $\delta: V \rightarrow \mathbb{N}$, we define the excess of a subset of vertices $U \subseteq V$ by

$$
\operatorname{exc}_{\delta}(U)=\left(\sum_{u \in U} \delta(u)\right)-\left|G_{U}\right|,
$$

where $\left|G_{U}\right|$ is the number of edges of $G$ having both endpoints in $U$. By definition, if $\delta$ is the outdegree sequence of an orientation $\mathcal{O}$, the sum $\sum_{u \in U} \delta(u)$ is the number of tails incident with vertices in $U$. From this number, exactly $\left|G_{U}\right|$ are part of edges with both endpoints in $U$. Hence, the excess $\operatorname{exc}_{\delta}(U)$ corresponds to the number of tails incident with vertices in $U$ in the cut defined by $U$. It is clear that if $\delta: V \rightarrow \mathbb{N}$ is an outdegree sequence, then the excess of $V$ is 0 and the excess of any subset $U \subseteq V$ is non-negative. In fact, the converse is also true: every function $\delta: V \rightarrow \mathbb{N}$ satisfying these two conditions is an outdegree sequence [20].

The following easy Lemma (whose proof is omitted) characterizes the reachability between vertices in a directed graphs in terms of outdegree sequences.

Lemma 1 Let $G=(V, E)$ be a graph and let $u, v$ be two vertices. Let $\mathcal{O}$ be an orientation of $G$ and let $\delta$ be its outdegree sequence. Then $v$ is reachable from $u$ if and only if there is no subset of vertices $U \subseteq V$ containing $u$ and not $v$ and such that $\operatorname{exc}_{\delta}(U)=0$.

Since reachability only depends on the outdegree sequence of the orientation, one can define an outdegree sequence $\delta$ to be $u$-connected or strongly connected if the $\delta$-orientations 
are. The $u$-connected outdegree sequences were considered in [24] in connection with the cycle/cocycle reversing system (see Subsection 8.1).

\subsection{The sandpile model}

The sandpile model is a dynamical system introduced in statistical physics in order to study self-organized criticality [1, 17]. It appeared independently in combinatorics as the chip firing game [4]. Recurrent configurations play an important role in the model: they correspond to configurations that can be observed after a long period of time. The recurrent configuration are also equivalent to the $G$-parking functions introduced by Shapiro and Postnikov in the study of certain quotient of the polynomial ring [39]. Despite its simplicity, the sandpile model displays interesting enumerative $[10,18,34]$ and algebraic properties $[12,19]$.

Let $G=(V, E)$ be a graph with a vertex $v_{0}$ distinguished as the root-vertex. A configuration of the sandpile model (or sandpile configuration for short) is a function $\mathcal{S}: V \rightarrow \mathbb{N}$, where $\mathcal{S}(v)$ represents the number of grains of sand on $v$. A vertex $v$ is unstable if $\mathcal{S}(v)$ is greater than or equal to its degree $\operatorname{deg}(v)$. An unstable vertex $v$ can topple by sending a grain of sand through each of the incident edges. This leads to the new sandpile configuration $\mathcal{S}^{\prime}$ defined by $\mathcal{S}^{\prime}(u)=\mathcal{S}(u)+\operatorname{deg}(u, v)$ for all $u \neq v$ and $\mathcal{S}^{\prime}(v)=\mathcal{S}(v)-\operatorname{deg}(v, *)$, where $\operatorname{deg}(u, v)$ is the number of edges with endpoints $u, v$ and $\operatorname{deg}(v, *)$ is the number of non-loop edges incident to $v$. We denote this transition by $\mathcal{S}{ }_{-\rightarrow}^{v} \mathcal{S}^{\prime}$. An evolution of the system is represented in Figure 4.

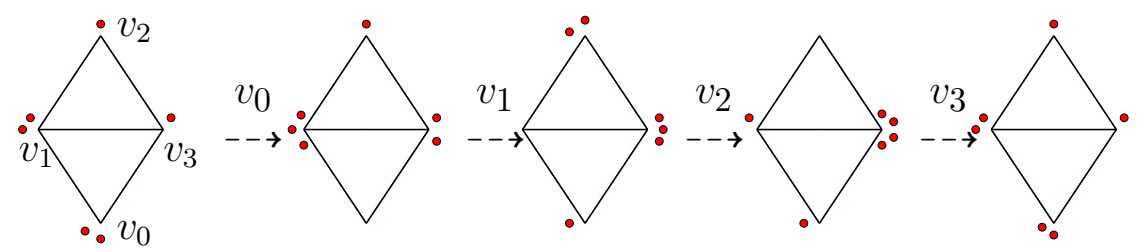

Figure 4: A recurrent configuration and the evolution rule.

A sandpile configuration is stable if every vertex $v \neq v_{0}$ is stable. A stable configuration $\mathcal{S}$ is recurrent if $\mathcal{S}\left(v_{0}\right)=\operatorname{deg}\left(v_{0}\right)$ and if there is a labeling of the $n$ vertices in $V$ by $v_{0}, v_{1}, \ldots, v_{n-1}$ such that $\mathcal{S}_{-\rightarrow}^{v_{0}} \mathcal{S}_{1} v_{-\rightarrow}^{v_{1}} \ldots{ }_{-\rightarrow}^{v_{n-1}} \mathcal{S}_{n}=\mathcal{S}$. This means that after toppling the root-vertex $v_{0}$, there is a valid sequence of toppling involving each vertex once that gets back to the initial configuration. For instance, the configuration at the left of Figure 4 is recurrent. Lastly, the level of a recurrent configuration $\mathcal{S}$ is given by: $\operatorname{level}(\mathcal{S})=\left(\sum_{v \in V} \mathcal{S}(v)\right)-|E|$. 


\section{A glimpse at the results}

\subsection{Tour of spanning trees and embedding-activities}

We first define the tour of spanning trees. Informally, the tour of a tree is a walk around the tree that follows internal edges and crosses external edges. A graphical representation of the tour is given in Figure 5 .
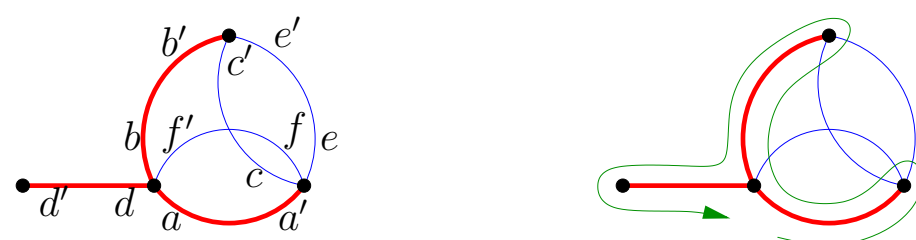

Tour of the tree

Figure 5: Intuitive representation of the tour of a spanning tree (indicated by thick lines).

Let $\mathcal{G}=(H, \sigma, \alpha)$ be an embedding of the graph $G=(V, E)$. Given a spanning tree $T$, we define the motion function $t$ on the set $H$ of half-edges by:

$$
t(h)=\mid \begin{array}{ll}
\sigma(h) & \text { if } h \text { is external, } \\
\sigma \alpha(h) & \text { if } h \text { is internal. }
\end{array}
$$

It was proved in [3] that $t$ is a cyclic permutation on $H$. For instance, for the embedded graph of Figure 5, the motion function is the cyclic permutation ( $a, e, f, c, a^{\prime}, f^{\prime}$, $\left.b, c^{\prime}, e^{\prime}, b^{\prime}, d, d^{\prime}\right)$. The cyclic order defined by the motion function $t$ on the set of half-edges is what we call the tour of the tree $T$.

We will now define the embedding-activities of spanning trees introduced in [3] in order to characterize the Tutte polynomial (see Theorem 4 below).

Definition 2 Let $\mathcal{G}=(H, \sigma, \alpha, h)$ be an embedded graph and let $T$ be a spanning tree. We define the $(\mathcal{G}, T)$-order on the set $H$ of half-edges by $h<t(h)<t^{2}(h)<\ldots<t^{|H|-1}(h)$, where $t$ is the motion function. (Note that the $(\mathcal{G}, T)$-order is a linear order on $H$ since $t$ is a cyclic permutation.) We define the $(\mathcal{G}, T)$-order on the edge set by setting $e=$ $\left\{h_{1}, h_{2}\right\}<e^{\prime}=\left\{h_{1}^{\prime}, h_{2}^{\prime}\right\}$ if $\min \left(h_{1}, h_{2}\right)<\min \left(h_{1}^{\prime}, h_{2}^{\prime}\right)$. (Note that this is also a linear order.)

Example: Consider the embedded graph $\mathcal{G}$ rooted on $a$ and the spanning tree $T$ represented in Figure 5. The $(\mathcal{G}, T)$-order on the half-edges is $a<e<f<c<a^{\prime}<f^{\prime}<$ $b<c^{\prime}<e^{\prime}<b^{\prime}<d<d^{\prime}$. Therefore, the $(\mathcal{G}, T)$-order on the edges is $\left\{a, a^{\prime}\right\}<\left\{e, e^{\prime}\right\}<$ $\left\{f, f^{\prime}\right\}<\left\{c, c^{\prime}\right\}<\left\{b, b^{\prime}\right\}<\left\{d, d^{\prime}\right\}$. 
Definition 3 Let $\mathcal{G}$ be a rooted embedded graph and $T$ be a spanning tree. We say that an external (resp. internal) edge is $(\mathcal{G}, T)$-active (or embedding-active if $\mathcal{G}$ and $T$ are clear from the context) if it is minimal for the $(\mathcal{G}, T)$-order in its fundamental cycle (resp. cocycle).

Example: In Figure 5, the $(\mathcal{G}, T)$-order on the edges is $\left\{a, a^{\prime}\right\}<\left\{e, e^{\prime}\right\}<\left\{f, f^{\prime}\right\}<$ $\left\{c, c^{\prime}\right\}<\left\{b, b^{\prime}\right\}<\left\{d, d^{\prime}\right\}$. Hence, the internal active edges are $\left\{a, a^{\prime}\right\}$ and $\left\{d, d^{\prime}\right\}$ and there is no external active edge. For instance, $\left\{e, e^{\prime}\right\}$ is not active since $\left\{a, a^{\prime}\right\}$ is in its fundamental cycle.

The following characterization of the Tutte polynomial was proved in [3].

Theorem 4 Let $\mathcal{G}$ be any rooted embedding of the connected graph $G$ (with at least one edge). The Tutte polynomial of $G$ is equal to

$$
T_{G}(x, y)=\sum_{T \text { spanning tree }} x^{\mathcal{I}(T)} y^{\mathcal{E}(T)},
$$

where the sum is over all spanning trees and $\mathcal{I}(T)$ (resp. $\mathcal{E}(T)$ ) is the number of $(\mathcal{G}, T)$ active internal (resp. external) edges.

Example: We represented the spanning trees of $K_{3}$ in Figure 6. If the embedding is rooted on the half-edge $a$, then the order on the edges is $a<c<b$ for the first two spanning trees and $a<b<c$ for the last one. Hence, the embedding-active edges are the one indicated by $a \star$ and the trees (taken from left to right) have respective contributions $x, x^{2}$ and $y$. Thus, by Theorem 4, the Tutte polynomial of $K_{3}$ is $T_{K_{3}}(x, y)=x^{2}+x+y$.
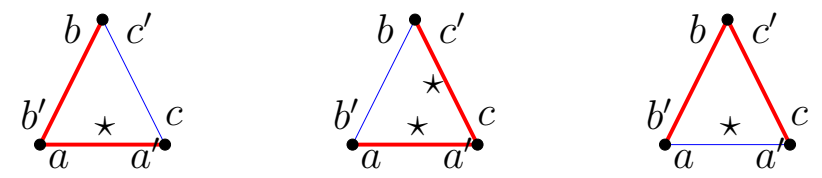

Figure 6: The embedding-activities of the spanning trees of $K_{3}$.

Note that the characterization (4) of the Tutte polynomial implies that the sum in the right-hand-side of (4) does not depend on the embedding, whereas the summands clearly depends on it. This characterization is reminiscent but inequivalent to the one given by Tutte in [43].

From now on we adopt the following conventions. If an embedding $\mathcal{G}$ and a spanning tree $T$ are clear from the context, the $(\mathcal{G}, T)$ order is denoted by $<$. If $F$ is a set of edges and $h$ is a half-edge, we say that $h$ is in $F$ if the edge $e$ containing $h$ is in $F$. A half-edge $h$ is said to be internal, external or $(\mathcal{G}, T)$-active if the edge $e$ is. 
We now make some elementary remarks about embedding-activities that will be useful throughout the paper.

Lemma 5 Let $\mathcal{G}$ be an embedded graph. Let $T$ be a spanning tree and let $e=\left\{h_{1}, h_{2}\right\}$ be an internal edge. Assume that $h_{1}<h_{2}$ (for the $(\mathcal{G}, T)$-order) and denote by $v_{1}$ and $v_{2}$ the endpoints of $h_{1}$ and $h_{2}$ respectively. Then, $v_{1}$ is the parent of $v_{2}$ in $T$. Moreover, the half-edges $h$ such that $h_{1}<h \leq h_{2}$ are the half-edges incident to a descendant of $v_{2}$.

Proof. Let $t$ be the motion function associated to the tree $T$ ( $t$ is defined by 3 ). We consider the subtrees $T_{1}$ and $T_{2}$ obtained from $T$ by deleting $e$ with the convention that $h_{1}$ is incident to $T_{1}$ and $h_{2}$ is incident to $T_{2}$. Let $h$ be any half-edge distinct from $h_{1}$ and $h_{2}$. By definition of $t$, the half-edges $h$ and $t(h)$ are incident to the same subtree $T_{i}$. Therefore, the $(\mathcal{G}, T)$-order is such that $h_{0}<l_{1}<\cdots<l_{i}<h_{1}<l_{1}^{\prime}<\cdots<l_{j}^{\prime}<h_{2}<l_{1}^{\prime \prime}<\cdots<l_{k}^{\prime \prime}$ where $l_{1}^{\prime}, \ldots, l_{j}^{\prime}, h_{2}$ are the half-edges incident with the subtree $T_{2}$ not containing the rootvertex $v_{0}$. Since the subtree $T_{2}$ does not contain $v_{0}$ its vertices are the descendants of $v_{2}$ in $T$.

Lemma 6 With the same assumption as in Lemma 5, let $e=\left\{h_{1}, h_{2}\right\}$ with $h_{1}<h_{2}$ be an internal edge and let $e^{\prime}=\left\{h_{1}^{\prime}, h_{2}^{\prime}\right\}$ with $h_{1}^{\prime}<h_{2}^{\prime}$ be an external edge.

- Then, $e$ is in the fundamental cycle of $e^{\prime}$ (equivalently, $e^{\prime}$ is in the fundamental cocycle of e) if and only if $h_{1}<h_{1}^{\prime}<h_{2}<h_{2}^{\prime}$ or $h_{1}^{\prime}<h_{1}<h_{2}^{\prime}<h_{2}$.

- Suppose that e is in the fundamental cycle of $e^{\prime}$ and denote by $v_{1}, v_{2}, v_{1}^{\prime}, v_{2}^{\prime}$ the endpoints of $h_{1}, h_{2}, h_{1}^{\prime}, h_{2}^{\prime}$ respectively. Recall that $v_{1}$ is the parent of $v_{2}$ in $T$ (Lemma 5) and that exactly one of the vertices $v_{1}^{\prime}, v_{2}^{\prime}$ is a descendant of $v_{2}$. If $e<e^{\prime}$, then $v_{1}^{\prime}$ is the descendant of $v_{2}$, else it is $v_{2}^{\prime}$.

\section{Proof.}

- Let $V_{2}$ be the set of descendants of $v_{2}$. Recall that the edge $e^{\prime}$ is in the fundamental cocycle of $e$ if and only if it has one endpoint in $V_{2}$ and the other in $\overline{V_{2}}$. By Lemma 5 , this is equivalent to the fact that exactly one of the half-edges $h_{1}^{\prime}, h_{2}^{\prime}$ is in $\left\{h^{\prime}: h_{1}<h^{\prime} \leq h_{2}\right\}$. Thus, $e^{\prime}$ is in the fundamental cocycle of $e$ if and only if $h_{1}<h_{1}^{\prime}<h_{2}<h_{2}^{\prime}$ or $h_{1}^{\prime}<h_{1}<h_{2}^{\prime}<h_{2}$.

- Suppose that $e$ is in the fundamental cycle of $e^{\prime}$. By the preceding point, $e<e^{\prime}$ implies $h_{1}<h_{1}^{\prime}<h_{2}<h_{2}^{\prime}$. In this case, $h_{1}^{\prime}$ is incident to a descendant of $v_{2}$ by Lemma 5 . Similarly, $e^{\prime}<e$ implies $h_{1}^{\prime}<h_{1}<h_{2}^{\prime}<h_{2}$, hence $h_{2}^{\prime}$ is incident to a descendant of $v_{2}$.

Lemma 7 An external edge $e^{\prime}=\left\{h_{1}^{\prime}, h_{2}^{\prime}\right\}$ with $h_{1}^{\prime}<h_{2}^{\prime}$ is $(\mathcal{G}, T)$-active if and only if the endpoint of $h_{1}^{\prime}$ is an ancestor of the endpoint of $h_{2}^{\prime}$. 
Proof. Denote by $v_{1}^{\prime}$ and $v_{2}^{\prime}$ the endpoints of $h_{1}^{\prime}$ and $h_{2}^{\prime}$ respectively.

- Suppose $v_{1}^{\prime}$ is an ancestor of $v_{2}^{\prime}$. We want to prove that $e^{\prime}$ is active. Let $e=\left\{h_{1}, h_{2}\right\}$ with $h_{1}<h_{2}$ be an internal edge in the fundamental cycle of $e^{\prime}$. The edge $e$ is in the path of $T$ between $v_{1}^{\prime}$ and $v_{2}^{\prime}$. Denote by $v_{1}$ and $v_{2}$ the endpoints of $h_{1}$ and $h_{2}$ respectively. Recall that $v_{1}$ is the parent of $v_{2}$ (Lemma 5). Since $v_{2}^{\prime}$ is a descendant of $v_{2}$, we have $e^{\prime}<e$ by Lemma 6 . The edge $e^{\prime}$ is less than any edge in its fundamental cycle hence it is $(\mathcal{G}, T)$-active.

- Suppose that $v_{1}^{\prime}$ is not an ancestor of $v_{2}^{\prime}$. Then the edge $e=\left\{h_{1}, h_{2}\right\}$ with $h_{1}<h_{2}$ linking $v_{1}^{\prime}$ to its parent in $T$ is in the fundamental cycle of $e^{\prime}$. If we denote by $v_{1}$ and $v_{2}$ the endpoints of $h_{1}$ and $h_{2}$ respectively, we get $v_{2}=v_{1}^{\prime}$ by Lemma 5. Since the endpoint $v_{1}^{\prime}$ of $h_{1}^{\prime}$ is a descendant of the endpoint $v_{2}$ of $h_{2}$, we get $e<e^{\prime}$ by Lemma 6 . Thus, $e^{\prime}$ is not $(\mathcal{G}, T)$-active.

\subsection{A mapping from spanning trees to orientations and some related bijections}

We now take a glimpse at the results to be developed in the following sections. In order to present these results, we define a mapping $\Phi$ from spanning trees to orientations. The mapping $\Phi$ will be extended into a bijection between subgraphs and orientations in Section 5. Related to the mapping $\Phi$, we define two other mappings $\Gamma$ and $\Lambda$ on the set of spanning trees. The mapping $\Gamma$ is a bijection between spanning trees and root-connected outdegree sequences while $\Lambda$ is a bijection between spanning trees and recurrent sandpile configurations.

Consider an embedded graph $\mathcal{G}=\left(H, \sigma, \alpha, h_{0}\right)$ and a spanning tree $T$. Recall that the tour of $T$ defines a linear order, the $(\mathcal{G}, T)$-order, on $H$ for which the root $h_{0}$ is the least element. We associate with the spanning tree $T$ the orientation $\mathcal{O}_{T}$ of $\mathcal{G}$ defined by:

For any edge $e=\left\{h_{1}, h_{2}\right\}$ with $h_{1}<h_{2}, \mathcal{O}_{T}(e)=\mid \begin{array}{ll}\left(h_{1}, h_{2}\right) & \text { if } e \text { is internal, } \\ \left(h_{2}, h_{1}\right) & \text { if } e \text { is external. }\end{array}$

This definition is illustrated in Figure 7 (left).

Observe that the spanning tree $T$ is oriented from its root-vertex $v_{0}$ to its leaves in $\mathcal{O}_{T}$. Indeed, it is clear from the definitions and Lemma 5 that every internal edge is oriented from parent to child. This property implies that for every spanning tree $T$ the orientation $\mathcal{O}_{T}$ is $v_{0}$-connected.

The mapping $\Phi: T \mapsto \mathcal{O}_{T}$ from spanning trees to $v_{0}$-connected orientations is not bijective. However, it is injective and in Section 5 we will extend it into a bijection between subgraphs and orientations. For the time being, let us observe (the proof will be given in Section 5) that the tree $T$ can be recovered from the orientation $\mathcal{O}_{T}$ by the following procedure: 

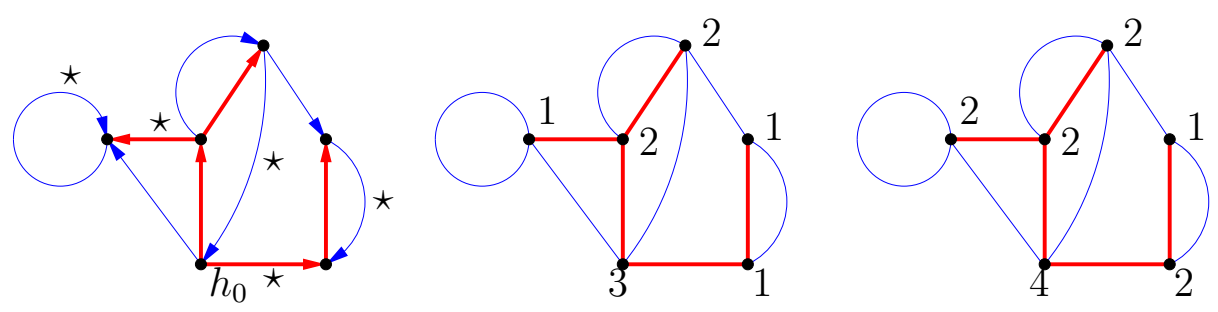

Figure 7: Left: Orientation $\mathcal{O}_{T}$ associated to the spanning tree $T$ (indicated by thick lines) and active edges (indicated by a star). Middle: outdegree sequence $\Gamma(T)$. Right: recurrent sandpile configuration $\Lambda(T)$.

\section{Procedure Construct-tree:}

- Initialize the current half-edge $h$ to be the root $h_{0}$. Initialize the tree $T$ and the set of visited arcs $F$ to be empty.

\section{- Core:}

C1: If the edge $e$ containing $h$ is not in $F$ and $h$ is a tail then add $e$ to $F$ and to $T$.

C2: Move to the next half-edge around $T$ : if $e$ is in $T$, then set the current half-edge $h$ to be $\sigma \alpha(h)$, else set it to be $\sigma(h)$.

Repeat steps $\mathbf{C 1}$ and $\mathbf{C 2}$ until the current half-edge $h$ is $h_{0}$.

- Return the tree $T$.

In the procedure Construct-tree we keep track of the set $F$ of edges already visited. The decision of adding an edge $e$ to the tree $T$ or not is taken when $e$ is visited for the first time. The principle of procedure Construct-tree, which constructs the tree $T$ while making its tour, will appear again in the next sections.

Building on the mapping $\Phi: T \mapsto \mathcal{O}_{T}$, we define two mappings $\Gamma$ and $\Lambda$.

Definition 8 Let $\mathcal{G}$ be an embedded graph. The mapping $\Gamma$ associates with any spanning tree $T$ the outdegree sequence of the orientation $\mathcal{O}_{T}$.

Definition 9 Let $\mathcal{G}$ be an embedded graph and let $V$ be the vertex set. The mapping $\Lambda$ associates with any spanning tree $T$ the sandpile configuration $\mathcal{S}_{T}: V \mapsto \mathbb{N}$, where $\mathcal{S}_{T}(v)$ is the number of tails plus the number of external $(\mathcal{G}, T)$-active heads incident to $v$ in the orientation $\mathcal{O}_{T}$.

The mappings $\Gamma$ and $\Lambda$ are illustrated in Figure 7 .

As observed above, the orientation $\mathcal{O}_{T}$ is always $v_{0}$-connected hence the image of any spanning tree by the mapping $\Gamma$ is a $v_{0}$-connected outdegree sequence. We shall prove in Section 6 that $\Gamma$ is a bijection between spanning tree and $v_{0}$-connected outdegree sequences. We will also show how to extend it into a bijection between forests and outdegree sequences. Regarding the mapping $\Lambda$, we shall prove in Section 7 that it is a bijection between spanning trees and recurrent sandpile configurations. Moreover, the number of 
external $(\mathcal{G}, T)$-active edges is easily seen to be the level of the configuration $\Lambda(T)$. This gives a new bijective proof of a result by Merino linking external activities to the level of recurrent sandpile configurations [10, 34].

The two mappings $\Gamma$ and $\Lambda$ coincide on internal trees, that is, trees that have external activity 0 . Thus, the mapping $\Gamma \circ \Lambda^{-1}$ is a bijection between recurrent sandpile configurations and $v_{0}$-connected outdegree sequences that leaves the configurations at level 0 unchanged. This answers a problem raised by Gioan [24]. As an illustration we represent the 5 spanning trees of a graph in Figure 8 and their image by the mappings $\Phi, \Gamma$ and $\Lambda$ (the first two spanning trees are internal).

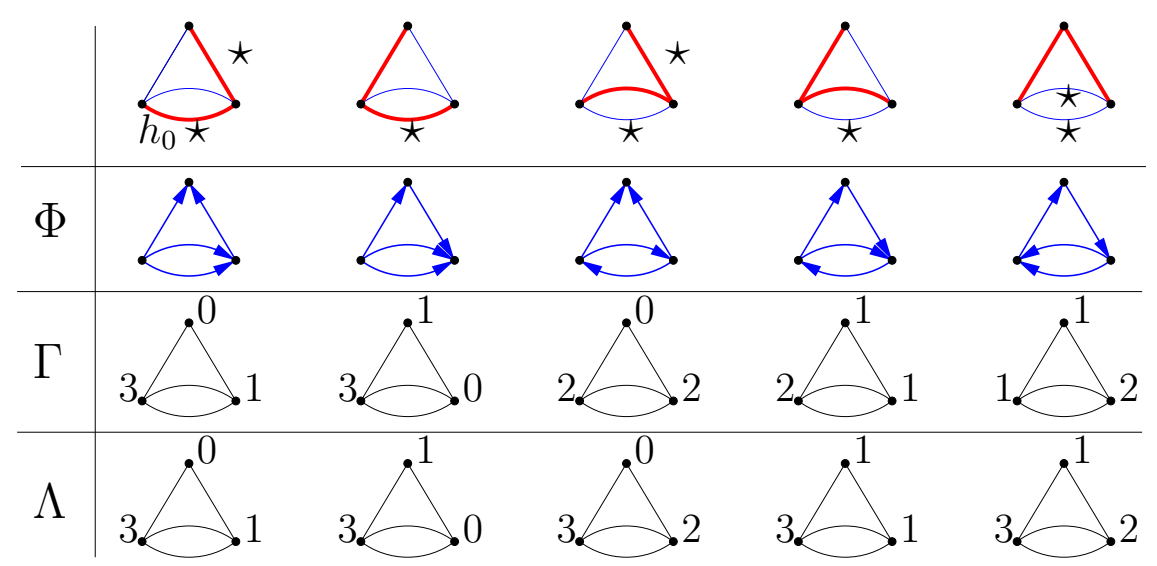

Figure 8: Spanning trees (embedding-active edges are indicated by a star) and their image by the mappings $\Phi, \Gamma$ and $\Lambda$.

We now highlight a relation (to be exploited in Section 6) between the embeddingactivities of the spanning tree $T$ and the acyclicity or strong connectivity of the associated orientation $\mathcal{O}_{T}$.

Lemma 10 Let $\mathcal{G}$ be an embedded graph ant let $T$ be a spanning tree. The fundamental cycle (resp. cocycle) of an external (resp. internal) edge $e$ is $\mathcal{O}_{T}$-directed if and only if e is $(\mathcal{G}, T)$-active.

Lemma 10 is illustrated by Figures 9 and 10. From this lemma we deduce that if $\mathcal{O}_{T}$ is acyclic (resp. strongly connected) then $T$ is internal (resp. external), that is, has no external (resp. internal) active edge. In fact, we shall prove in Section 6 that the converse is true: if the tree $T$ is internal (resp. external), then the orientation $\mathcal{O}_{T}$ is acyclic (resp. strongly connected). For instance, in Figure 8 the first two (resp. last two) spanning trees are internal (resp. external) and the corresponding orientations are acyclic (resp. strongly connected). 

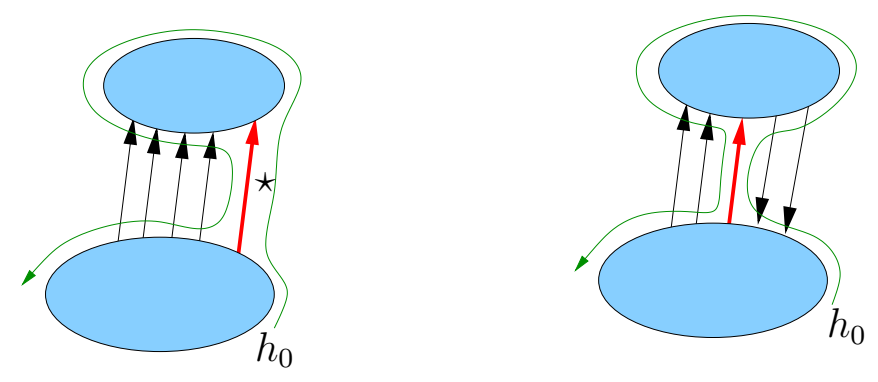

Figure 9: Fundamental cocycles of an active internal edge (left) and of a non-active internal edge (right).
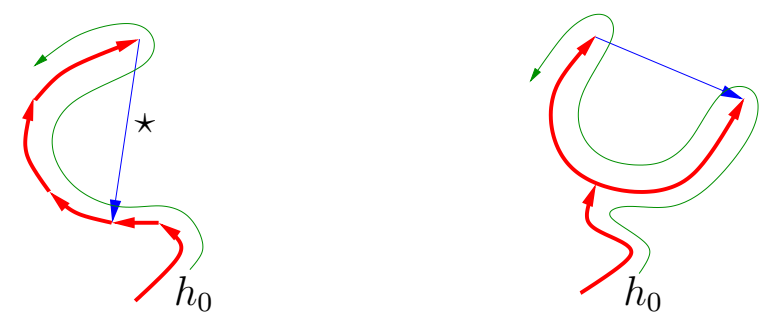

Figure 10: Fundamental cycles of an active external edge (left) and of a non-active external edge (right).

Proof. Consider an edge $e=\left\{h_{1}, h_{2}\right\}$ with $h_{1}<h_{2}$ and denote by $v_{1}$ and $v_{2}$ the endpoints of $h_{1}$ and $h_{2}$ respectively.

- Suppose that $e$ is internal. We want to prove that the fundamental cocycle $D$ of $e$ is directed if and only if $e$ is $(\mathcal{G}, T)$-active. Recall that $v_{1}$ is the parent of $v_{2}$ by Lemma 5 . Let $V_{2}$ be the set of descendants of $v_{2}$. Recall that $D$ is the cocycle defined by $V_{2}$. By definition, the $\operatorname{arc} \mathcal{O}_{T}(e)$ is directed toward $v_{2} \in V_{2}$. By Lemma 6 , for all edge $e^{\prime}=\left\{h_{1}^{\prime}, h_{2}^{\prime}\right\}$ with $h_{1}^{\prime}<h_{2}^{\prime}$ in $D-e$, the arc $\mathcal{O}_{T}\left(e^{\prime}\right)=\left(h_{2}^{\prime}, h_{1}^{\prime}\right)$ is directed toward $V_{2}$ if and only if $e<e^{\prime}$. Therefore, the fundamental cocycle $D$ is directed if and only if $e$ is minimal in $D$, that is, if $e$ is $(\mathcal{G}, T)$-active.

- Suppose that $e$ is external. We want to prove that the fundamental cycle $C$ of $e$ is directed if and only if $e$ is $(\mathcal{G}, T)$-active. Recall that $C-e$ is the path in $T$ between $v_{1}$ and $v_{2}$. Since $\mathcal{O}_{T}(e)$ is directed toward $v_{1}$, the cycle $C$ is directed if and only if the path $C-e$ is directed from $v_{1}$ to $v_{2}$. Since every edge in $C-e \subseteq T$ is directed from parent to child (Lemma 5), the cycle $C$ is directed if and only if $v_{1}$ is an ancestor of $v_{2}$. This is precisely the characterization of external $(\mathcal{G}, T)$-active edges given by Lemma 7 .

Up to this point we have considered mappings defined on the set of spanning trees. In order to extend these mappings to general subgraphs we will associate a spanning tree to every subgraph. This is the task of the next section. 


\section{A partition of the set of subgraphs}

In this section we define a partition of the set of subgraphs for any embedded graph. Each part of this partition is associated with a spanning tree. Our partition is closely related to the notion of embedding-activities.

Let $\mathcal{G}$ be an embedded graph. Given a spanning tree $T$, we consider the set of subgraphs that can be obtained from $T$ by removing some internal $(\mathcal{G}, T)$-active edges and adding some external $(\mathcal{G}, T)$-active edges. Observe that this set is an interval in the boolean lattice of the subgraphs of $\mathcal{G}$ (i.e. subsets of edges). We call tree-interval and denote by $\left[T^{-}, T^{+}\right]$the set of subgraphs obtained from a spanning tree $T$. We represent the tree-intervals corresponding to each of the 5 spanning trees of the embedded graph in Figure 11. We now state the main result of this section.

Theorem 11 Let $G=(V, E)$ be a graph and let $\mathcal{G}$ be an embedding of $G$. The treeintervals form a partition of the set of subgraphs of $G$ :

$$
2^{E}=\biguplus_{\text {T spanning tree }}\left[T^{-}, T^{+}\right]
$$

where the disjoint union is over all spanning trees of $G$.

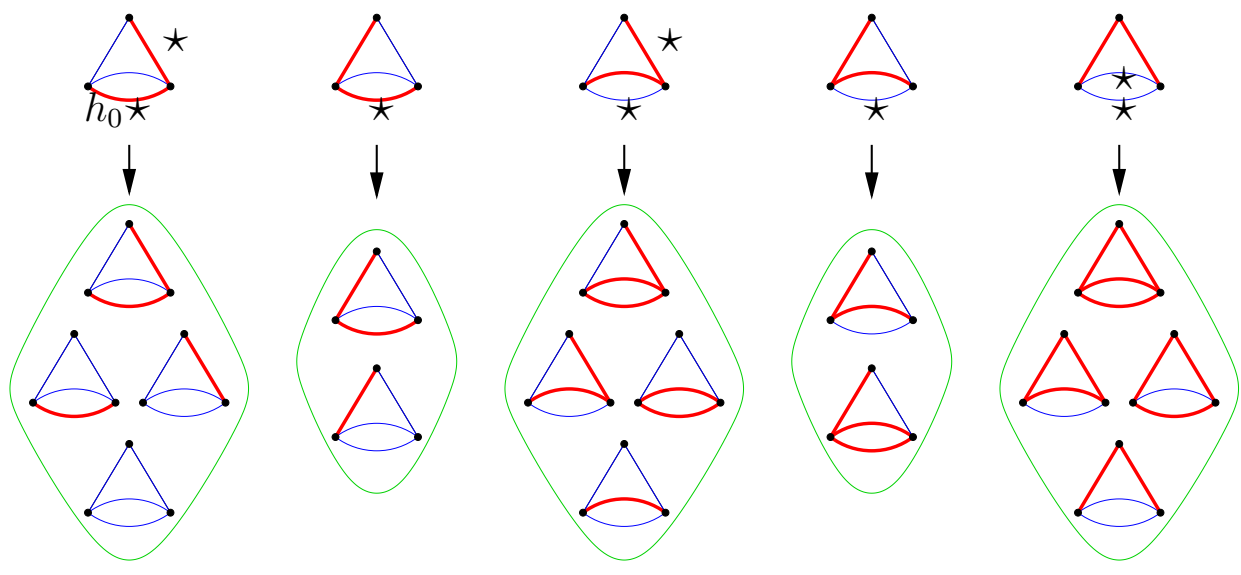

Figure 11: The tree-intervals corresponding to each spanning tree. The active edges are indicated by $\mathrm{a} \star$.

The counterpart of Theorem 11 is known for the notion of (internal and external) activities defined by Tutte in [43]. This property has been used to extract information about the Tutte polynomial in $[2,14,27]$. In fact, Theorem 11 and its counterpart are both consequences of a more general result proved by Gordon and McMahon [26] and which relies on the notion of a computation tree. We now recall this notion. 
For a graph $G$ and an edge $e$, we denote by $G_{\backslash e}$ and $G_{/ e}$ respectively the graphs obtained from $G$ by deleting and by contracting the edge e. A computation tree for a graph $G$ is a tree whose vertices are labeled by some minors of $G$ and which obeys the following inductive rules. If $G$ has no edge, then the computation tree is made of a single vertex labelled by $G$. Otherwise, a computation tree for the graph $G$ is any tree made of a root-vertex labelled by $G$ and joined to either

- two subtrees, one being a computation tree of $G_{\backslash e}$ and the other being a computation tree of $G_{/ e}$, where $e$ is any edge of $G$ which is neither a loop nor an isthmus,

- or one subtree which is a computation tree of $G_{\backslash e}$, where $e$ is any loop of $G$, - or one subtree which is a computation tree of $G_{/ e}$ where $e$ is any isthmus of $G$.

It is easy to see that for any computation tree $\mathbf{T}$ of a connected graph $G$, the leaves of $\mathbf{T}$ are in one-to-one correspondence with the spanning trees of $G$ (the leaf $l$ is in correspondence with the spanning tree made of the edges which are contracted on the path of the computation tree $\mathbf{T}$ from the root to the leaf $l$ ). Given a spanning tree $T$ of $G$ corresponding to a leaf $l$ of $\mathbf{T}$, one says that an internal (resp. external) edge is ( $\mathbf{T}, T)$-active if this edge is contracted as an isthmus (resp. deleted as a loop) on the path of the computation tree $\mathbf{T}$ going from the root-vertex to the leaf $l$. Proposition 2.7 of [26] states that for any computation-tree $\mathbf{T}$ of a graph $G=(V, E)$, the counterpart of Theorem 11 holds, that is,

$$
2^{E}=\biguplus_{T \text { spanning tree }}[T \backslash \operatorname{int}(\mathbf{T}, T), T \cup \operatorname{ext}(\mathbf{T}, T)]
$$

where the disjoint union is over all spanning trees of $G$ and $\operatorname{int}(\mathbf{T}, T)(\operatorname{resp} \operatorname{ext}(\mathbf{T}, T))$ is the set of internal (resp. external) $(\mathbf{T}, T)$-active edges.

In order to prove Theorem 11, it only remains to show the following lemma.

Lemma 12 Let $G$ be a connected graph. For any embedding $\mathcal{G}$ of $G$, there exists a computation tree $\boldsymbol{T}(\mathcal{G})$ of $G$ such that for any spanning tree $T$ of $G$ the $(\mathcal{G}, T)$ - and $(\boldsymbol{T}(\mathcal{G}), T)$-active edges coincide.

Proof. Informally, the computation tree $\mathbf{T}(\mathcal{G})$ is obtained by recursively considering the edge $e$ of $\mathcal{G}$ preceding the root-edge around the root-vertex. In order to make this definition precise, we need to define the deletion and contraction of edges in an embedded graph. We represent the result of deleting and contracting an edge in Figure 12.

Let $\mathcal{G}=\left(H, \sigma, \alpha, h_{0}\right)$ be an embedding of a graph $G$ and let $e=\left\{h_{1}, h_{2}\right\}$ be an edge. We assume that $\mathcal{G}$ has at least two edges (otherwise $\mathcal{G}_{\backslash e}$ or $\mathcal{G}_{/ e}$ is the unique embedding of the graph made of one vertex and no edge) and consider the set of half-edges $H^{\prime}=H \backslash\left\{h_{1}, h_{2}\right\}$, the involution $\alpha^{\prime}$ which is the restriction of $\alpha$ to $H^{\prime}$, and the permutation $\sigma^{\prime}$ (resp. $\phi^{\prime}$ ) whose cycles are obtained from the cycles of $\sigma$ (resp. $\phi=\alpha \sigma)$ by erasing $h_{1}$ and $h_{2}$. If $e$ is not an isthmus, then $\mathcal{G}_{{ }_{e}}$ denotes the embedding $\left(H^{\prime}, \sigma^{\prime}, \alpha^{\prime}, h_{0}^{\prime}\right)$ of $G_{\backslash e}$, where $h_{0}^{\prime}=\sigma^{k}\left(h_{0}\right)$ with $k=0,1$ or 2 the least non-negative integer such that $\sigma^{k}\left(h_{0}\right) \neq h_{1}, h_{2}$. Similarly, if $e$ 

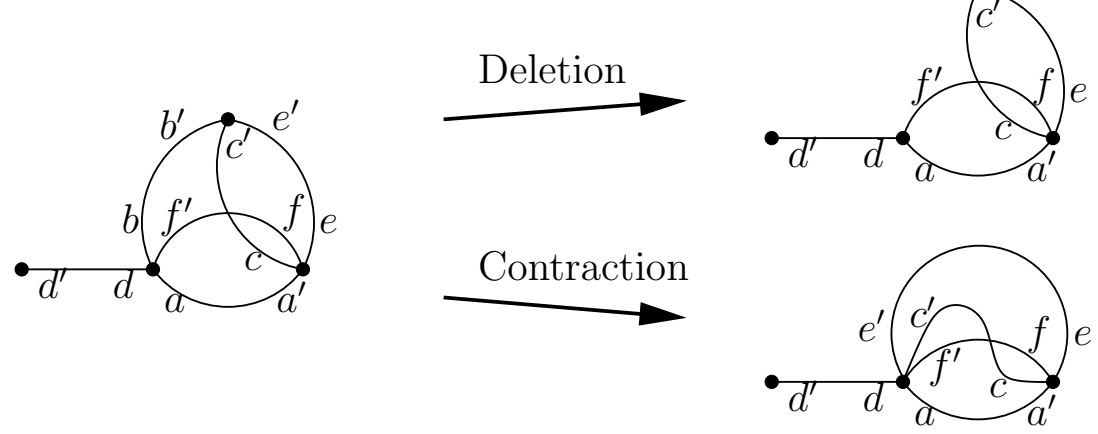

Figure 12: Deletion and contraction of the edge $e=\left\{b, b^{\prime}\right\}$.

is not a loop, then $\mathcal{G}_{/ e}$ denotes the embedding $\left(H^{\prime}, \sigma^{\prime}, \alpha^{\prime \prime}, h_{0}^{\prime \prime}\right)$ of $G_{/ e}$, where $\sigma^{\prime \prime}=\alpha \phi^{\prime}$ and $h_{0}^{\prime \prime}=\phi^{k}\left(h_{0}\right)$ with $k=0,1$ or 2 the least non-negative integer such that $\phi^{k}\left(h_{0}\right) \neq h_{1}, h_{2}$.

We now define a computation tree $\mathbf{T}(\mathcal{G})$ associated to the embedding $\mathcal{G}=\left(H, \sigma, \alpha, h_{0}\right)$. If $\mathcal{G}$ has no edge, then the computation tree $\mathbf{T}(\mathcal{G})$ is made of a single vertex labelled by $G$. Otherwise, we consider the edge $e$ of $G$ containing the half-edge $\sigma^{-1}\left(h_{0}\right)$ and define the computation $\mathbf{T}(\mathcal{G})$ to be the tree made of a root-vertex labelled by $G$ joined to either - the computation trees $\mathbf{T}\left(\mathcal{G}_{\backslash e}\right)$ and $\mathbf{T}\left(\mathcal{G}_{/ e}\right)$, if $e$ is neither a loop nor an isthmus,

- or the computation tree $\mathbf{T}(\mathcal{G} \backslash e)$, if $e$ is a loop of $G$,

- or the computation tree $\mathbf{T}\left(\mathcal{G}_{/ e}\right)$, if $e$ is an isthmus of $G$.

We want to show that for any spanning tree $T$ of $\mathcal{G}$, the $(\mathcal{G}, T)$ - and $(\mathbf{T}(\mathcal{G}), T)$-active edges coincide. We proceed by induction on the number of edges of the graph $G$. If $G$ has no edge, the property holds. Consider now an embedded graph $\mathcal{G}=\left(H, \sigma, \alpha, h_{0}\right)$ with at least one edge. Let $e$ be the edge containing the half-edge $\sigma^{-1}\left(h_{0}\right)$ and let $T$ be a spanning tree of $G$. It was shown in [3] that the edge $e$ is $(\mathcal{G}, T)$-active if and only if $e$ is a loop or an isthmus. Moreover, if $e$ is internal (resp. external), then any other edge of $G$ is $(\mathcal{G}, T)$ active if and only if it is $(\mathcal{G} \backslash e, T)$-active (resp. $\left(\mathcal{G}_{/ e}, T_{/ e}\right)$-active). The same properties are obviously true for $(\mathbf{T}(\mathcal{G}), T)$-activities: the edge $e$ is $(\mathbf{T}(\mathcal{G}), T)$-active if and only if $e$ is a loop or an isthmus; moreover if $e$ is internal (resp. external), then any other edge is $(\mathbf{T}(\mathcal{G}), T)$-active if and only if it is $(\mathbf{T}(\mathcal{G} \backslash e), T)$-active $\left(\operatorname{resp} .\left(\mathbf{T}\left(\mathcal{G}_{/ e}\right), T_{/ e}\right)\right.$-active). By the induction hypothesis, the $\left(\mathcal{G}_{\mid e}, T\right)$ - and $\left(\mathcal{G}_{/ e}, T_{/ e}\right)$ - activities coincide respectively with the $(\mathbf{T}(\mathcal{G} \backslash e), T)$ - and $\left(\mathbf{T}\left(\mathcal{G}_{/ e}\right), T_{/ e}\right)$ - activities, therefore $(\mathcal{G}, T)$ - and $(\mathbf{T}(\mathcal{G}), T)$-activities also coincide.

We will now prove some properties of the subgraphs in the tree-interval $\left[T^{-}, T^{+}\right]$and comment on Theorem 11.

Lemma 13 Let $\mathcal{G}$ be an embedded graph and let $T$ be a spanning tree. Let $e$ be an internal (resp. external) $(\mathcal{G}, T)$-active edge. The fundamental cocycle (resp. cycle) of e is contained in $\bar{S}+e$ (resp. $S+e)$ for any subgraph $S$ in $\left[T^{-}, T^{+}\right]$. 
Proof. If $e$ is internal and $(\mathcal{G}, T)$-active, no edge in its fundamental cocycle $D$ is $(\mathcal{G}, T)$ active (since their fundamental cycle contains $e$ ). Since no edge of $D-e$ is in $T$ nor is $(\mathcal{G}, T)$-active, none is in $S$. Hence, $D \subseteq \bar{S}+e$. Similarly, if $e$ is external $(\mathcal{G}, T)$-active, its fundamental cycle is contained in $S+e$.

Lemma 14 Let $\mathcal{G}$ be an embedded graph. Let $T$ be a spanning tree and let $S$ be a subgraph in $\left[T^{-}, T^{+}\right]$having $c(S)$ connected components. Then $c(S)-1$, (resp. e $\left.(S)+c(S)-|V|\right)$ is the number of edges in $\bar{S} \cap T$ (resp. $S \cap \bar{T}$ ).

Proof. Consider any subgraph $S$ in $\left[T^{-}, T^{+}\right]$. By Lemma 13 , removing an internal $(\mathcal{G}, T)$ active edge from $S$ increases $c(S)$ by one and leaves $e(S)+c(S)$ unchanged. Similarly, adding an external $(\mathcal{G}, T)$-active edge to $S$ leaves $c(S)$ unchanged and increases $e(S)+c(S)$ by one. Moreover, $c(T)-1=0$ and $e(T)+c(T)-|V|=0$. Therefore, Lemma 14 holds for every subgraph $S$ in $\left[T^{-}, T^{+}\right]$by induction on the number of edges in $S \triangle T$.

By Lemma 14, the connected subgraphs in $\left[T^{-}, T^{+}\right]$are the subgraphs in the interval $\left[T, T^{+}\right]$(the subgraphs obtained from $T$ by adding some external $(\mathcal{G}, T)$-active edges). Similarly, the forests in $\left[T^{-}, T^{+}\right]$are the subgraphs in the interval $\left[T^{-}, T\right]$ (the subgraphs obtained from $T$ by removing some internal $(\mathcal{G}, T)$-active edges). These properties are illustrated in Figure 13.

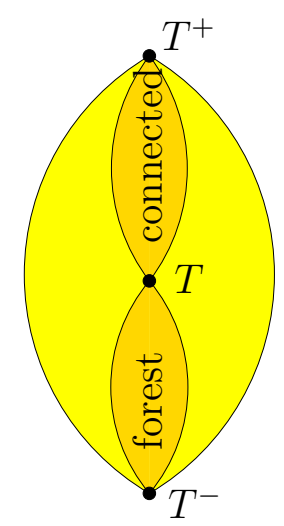

Figure 13: The tree-interval $\left[T^{-}, T^{+}\right]$, the sub-interval $\left[T, T^{+}\right]$of connected subgraphs and the sub-interval $\left[T^{-}, T\right]$ of forests.

Theorem 11 constitutes the key link between the subgraph expansion (1) and spanning tree expansion (2) of the Tutte polynomial. Indeed, given Lemma 14, one gets

$$
\sum_{S \in\left[T^{-}, T^{+}\right]}(x-1)^{c(S)-1}(y-1)^{e(S)+c(S)-|V|}=(x-1+1)^{\mathcal{I}(T)}(y-1+1)^{\mathcal{E}(T)}=x^{\mathcal{I}(T)} y^{\mathcal{E}(T)},
$$


where $\mathcal{I}(T)$ (resp. $\mathcal{E}(T)$ ) is the number of internal (resp. external) $(\mathcal{G}, T)$-active edges. Summing over all spanning trees gives the identity:

$$
\sum_{S \text { subgraph }}(x-1)^{c(S)-1}(y-1)^{e(S)+c(S)-|V|}=\sum_{T \text { spanning tree }} x^{\mathcal{I}(T)} y^{\mathcal{E}(T)} .
$$

Remark. As observed in [27], the partition of the set of subgraphs gives several other expansions of the Tutte polynomial. For instance, the tree-intervals can be partitioned into forest-intervals. The forest-interval of a forest $F$ in $\left[T^{-}, T^{+}\right]$is the set $\left[F, F^{+}\right]$of subgraphs obtained from $F$ by adding some external $(\mathcal{G}, T)$-active edges. Since

$$
\left[T^{-}, T^{+}\right]=\biguplus_{F \text { forest in }\left[T^{-}, T^{+}\right]}\left[F, F^{+}\right]
$$

the partition into tree-intervals given by Theorem 11 leads to a partition into forestintervals:

$$
2^{E}=\biguplus_{F \text { forest }}\left[F, F^{+}\right]
$$

Given Lemma 14, we get

$\sum_{S \in\left[F, F^{+}\right]}(x-1)^{c(S)-1}(y-1)^{e(S)+c(S)-|V|}=(x-1)^{c(F)-1}(y-1+1)^{\mathcal{E}(T)}=(x-1)^{c(F)-1} y^{\mathcal{E}(T)}$,

for any forest in $\left[T^{-}, T^{+}\right]$. Summing up over forests gives the forest expansion

$$
T_{G}(x, y)=\sum_{F \text { forest }}(x-1)^{c(F)-1} y^{\mathcal{E}(F)},
$$

where $\mathcal{E}(F)$ is the number of $(\mathcal{G}, T)$-active edges for the spanning tree $T$ such that $F \in\left[T^{-}, T^{+}\right]$. Let us mention that several alternative notions of external activities have been defined, each of which gives a forest expansion $[23,30]$ which can be used to obtain enumerative results about the Tutte polynomial $[22,23]$.

Before we close this section, we define some families of subgraphs counted by the evaluations $T_{G}(i, j), 0 \leq i, j \leq 2$ of the Tutte polynomial. Consider an embedded graph $\mathcal{G}$ and a spanning tree $T$. Recall that the spanning tree $T$ is said to be internal (resp. external) if it has no external (resp. internal) $(\mathcal{G}, T)$-active edge. For instance, among the spanning trees represented in Figure 11, the two first (resp. last) are internal (resp. external). We say that a subgraph $S$ in $\left[T^{-}, T^{+}\right]$is internal or external if the spanning tree $T$ is. The notion of internal subgraph is close to Whitney's notion of subgraphs without broken circuit [44]. Observe that by Lemma 14, any internal subgraph is a forest and any external subgraph is connected (the converse is, of course, false). In Figure 20, we represent the subgraphs of Figure 11 in each of the categories defined by the four criteria forest, internal, connected, external. An easy enumerative corollary of Lemma 14 and Theorem 11 is that the subgraphs in each of these categories are counted by the following evaluations of the Tutte polynomial: 


\begin{tabular}{|c|c|c|c|}
\hline & General & Connected & External \\
\hline General & $T_{G}(2,2)=2^{|E|}$ & $T_{G}(1,2)$ & $T_{G}(0,2)$ \\
\hline Forest & $T_{G}(2,1)$ & $T_{G}(1,1)$ & $T_{G}(0,1)$ \\
\hline Internal & $T_{G}(2,0)$ & $T_{G}(1,0)$ & $T_{G}(0,0)=0$ \\
\hline
\end{tabular}

Figure 14: Number of subgraphs in the categories defined by the criteria forest, internal, connected and external.

\section{A bijection between subgraphs and orientations}

In this section we define a bijection $\Phi$ between subgraphs and orientations. The bijection $\Phi$ is an extension of the correspondence $T \mapsto \mathcal{O}_{T}$ between spanning trees and orientations defined in Section 3. For instance, the image by $\Phi$ of the spanning tree $T$ and the image of a subgraph $S$ in $\left[T^{-}, T^{+}\right]$are shown in Figure 15.

Definition 15 Let $\mathcal{G}$ be an embedded graph. Let $T$ be a spanning tree and let $S$ be a subgraph in the tree-interval $\left[T^{-}, T^{+}\right]$. The orientation $\mathcal{O}_{S}=\Phi(S)$ is defined as follows. For any edge $e=\left\{h_{1}, h_{2}\right\}$ with $h_{1}<h_{2}$ (for the $(\mathcal{G}, T)$-order), the arc $\mathcal{O}_{S}(e)$ is $\left(h_{1}, h_{2}\right)$ if and only if - either $e$ is in $T$ and its fundamental cocycle contains no edge in the symmetric difference $S \triangle T$ - or if $e$ is not in $T$ and its fundamental cycle contains some edges in $S \triangle T$; the arc $\mathcal{O}_{S}(e)$ is $\left(h_{2}, h_{1}\right)$ otherwise.

Recall that a subgraph $S$ is in the tree-interval $\left[T^{-}, T^{+}\right]$if and only if every edge in the symmetric difference $S \triangle T$ is $(\mathcal{G}, T)$-active. Let $S$ be a subgraph in $\left[T^{-}, T^{+}\right]$and let $e$ be any edge of $\mathcal{G}$. We say that the $\operatorname{arc} \mathcal{O}_{S}(e)$ is reverse if $\mathcal{O}_{S}(e) \neq \mathcal{O}_{T}(e)$. Observe that the $\operatorname{arc} \mathcal{O}_{S}(e)$ is reverse if and only if the fundamental cycle or cocycle of $e$ (with respect to the spanning tree $T$ ) contains an edge of $S \triangle T$ (compare for instance the orientations $\mathcal{O}_{S}$ and $\mathcal{O}_{T}$ in Figure 15). In particular, Definition 15 of the mapping $\Phi$ extends the Definition 5 given for spanning trees in Section 3.
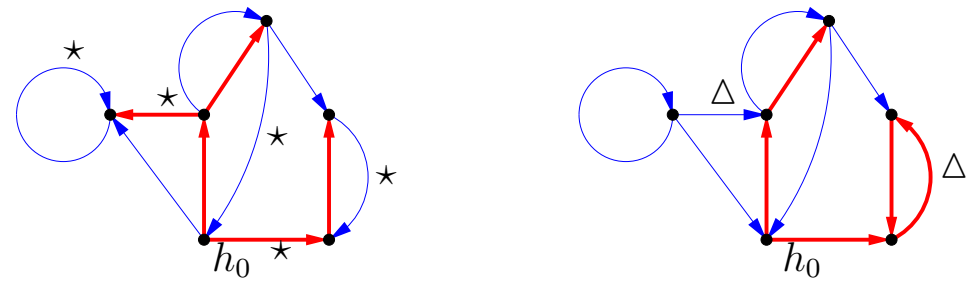

Figure 15: Left: the orientation $\mathcal{O}_{S}$ associated with a subgraph $S$ in $\left[T^{-}, T^{+}\right]$. Right: the orientation $\mathcal{O}_{T}$ associated with a spanning tree $T$. The active edges are indicated by a $\star$. The edges in the symmetric difference $S \triangle T$ are indicated by a $\triangle$.

The main result of this section is that the mapping $\Phi$ is a bijection between subgraphs and orientations. For instance, we have represented in Figure 16 the image by $\Phi$ of the subgraphs represented in Figure 11. 
Theorem 16 Let $\mathcal{G}$ be an embedded graph. The mapping $\Phi$ establishes a bijection between the subgraphs and the orientations of $G$.
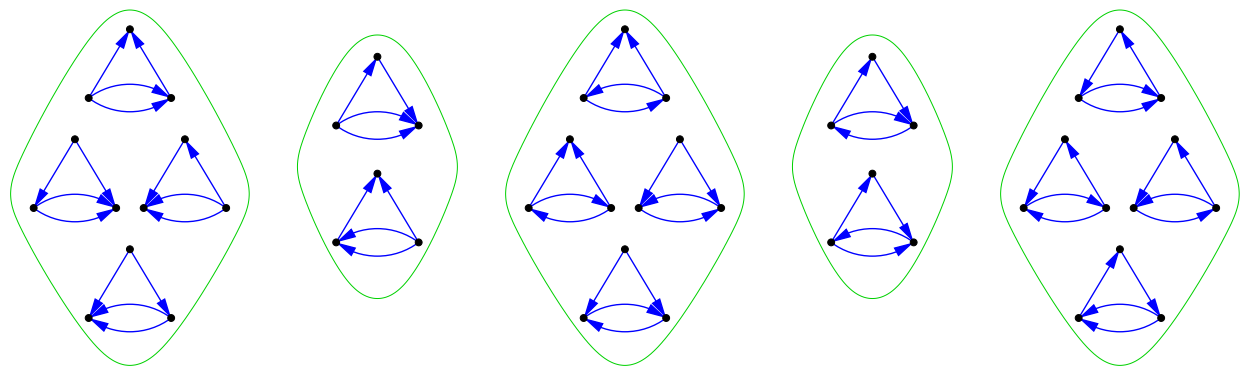

Figure 16: The image by $\Phi$ of the subgraphs in Figure 11.

In order to prove Theorem 16, we define a mapping $\Psi$ from orientations to subgraphs. We shall prove that $\Psi$ is the inverse of $\Phi$.

Definition 17 Let $\mathcal{G}$ be an embedded graph and let $\mathcal{O}$ be an orientation. We define the subgraph $S=\Psi(\mathcal{O})$ by the procedure described below. The procedure $\Psi$ visits the half-edges in sequential order. The set of visited edges is denoted by $F$ (and the set of unvisited one by $\bar{F}=E \backslash F)$. If $C$ is a set of edges that intersects the set $F$ of visited edges, we denote by $e_{\text {first }}(C)$ and $h_{\text {first }}(C)$ the first visited edge and half-edge of $C$ respectively $\left(e_{\text {first }}(C)\right.$ contains $\left.h_{\text {first }}(C)\right)$. In this case, $C$ is said to be tail-first if $h_{\text {first }}(C)$ is a tail and head-first otherwise.

Procedure $\Psi$ :

- Initialize the current half-edge $h$ to be the root $h_{0}$. Initialize the subgraph $S$, the tree $T$ and the set of visited edges $F$ to be empty.

- Core:

C1: If the edge e containing $h$ is not in $F$, then decide whether to add e to $S$ and to T:

- If $h$ is a tail, then

(a) If e is in a directed cycle $C \subseteq \bar{F}$, then add e to $S$ but not to $T$.

(b) If $e$ is in a head-first directed cocycle $D \nsubseteq \bar{F}$ such that for all directed cocycle $D^{\prime}$ with $e_{\text {first }}\left(D^{\prime}\right)=e_{\text {first }}(D)$ either $e \in D^{\prime}$ or $\left(D \triangle D^{\prime} \nsubseteq \bar{F}\right.$ and $\left.e_{\text {first }}\left(D \triangle D^{\prime}\right) \in D^{\prime}\right)$, then do not add e to $S$ nor to $T$.

(c) Else, add e to $S$ and to $T$.

- If $h$ is a head, then

$\left(a^{\prime}\right)$ If $e$ is in a directed cocycle $D \subseteq \bar{F}$, then add e to $T$ but not to $S$.

$\left(b^{\prime}\right)$ If $e$ is in a tail-first directed cycle $C \nsubseteq \bar{F}$ such that for all directed cycle $C^{\prime}$ with $e_{\text {first }}\left(C^{\prime}\right)=e_{\text {first }}(C)$ either $e \in C^{\prime}$ or $\left(C \triangle C^{\prime} \nsubseteq \bar{F}\right.$ and $\left.e_{\text {first }}\left(C \triangle C^{\prime}\right) \in C^{\prime}\right)$, then add $e$ to $S$ and to $T$.

$\left(c^{\prime}\right)$ Else, do not add e to $S$ nor to $T$. 
Add e to $F$.

C2: Move to the next half-edge around $T$ : if $e$ is in $T$, then set the current half-edge $h$ to be $\sigma \alpha(h)$, else set it to be $\sigma(h)$.

Repeat steps $\boldsymbol{C 1}$ and $\boldsymbol{C 2}$ until the current half-edge $h$ is $h_{0}$.

- Return the subgraph $S$.

Observe that the conditions $(a)$ and $(b)$ (resp. $\left(a^{\prime}\right)$ and $\left(b^{\prime}\right)$ ) in Procedure $\Psi$ are incompatible. We are now going to prove that $\Phi$ and $\Psi$ are inverse mappings.

Proposition 18 Let $\mathcal{G}$ be an embedded graph and let $S$ be a subgraph. The mapping $\Psi$ is well defined on the orientation $\Phi(S)$ (the procedure terminates) and $\Psi \circ \Phi(S)=S$.

Proposition 18 implies that the mapping $\Phi$ is injective. Since there are as many subgraphs and orientations $\left(2^{|E|}\right)$, it implies that $\Phi$ is bijective and that $\Psi$ and $\Phi$ are inverse mappings. The rest of this section is devoted to the proof of proposition 18. Observe that $\Psi$ is a variation on the procedure Construct-tree presented in Section 3. The difference lies in the extra Conditions $(a),(b),\left(a^{\prime}\right),\left(b^{\prime}\right)$ which are now needed in order to cope with reverse edges. In Lemmas 19 to 23 we express some properties characterizing reverse edges.

We first need some definitions. Let $\mathcal{G}$ be an embedded graph and $\mathcal{O}$ be an orientation. Suppose that the edges and half-edges of $\mathcal{G}$ are linearly ordered. For any set of edges $C$, we denote by $e_{\min }(C)$ and $h_{\min }(C)$ the minimal edge and half-edge of $C$ respectively. We say that $C$ is tail-min if $h_{\min }(C)$ is a tail and head-min otherwise. A directed cycle (resp. cocycle) is tight if any directed cycle (resp. cocycle) $C^{\prime} \neq C$ with $e_{\min }\left(C^{\prime}\right)=e_{\min }(C)$ satisfies $e_{\min }\left(C \triangle C^{\prime}\right) \in C^{\prime}$. For instance, if the edges of the graph in Figure 17 are ordered by $a<b<c<d<e<f<g$, the directed cycles $(a, h, g, f, e, c)$ and $(b, g, f, e, c)$ are tight whereas $(a, h, g, d, c)$ is not.

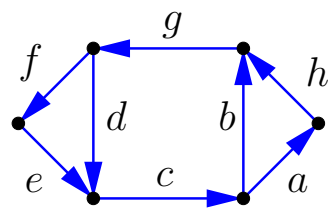

Figure 17: The directed cycles $(a, h, g, f, e, c)$ and $(b, g, f, e, c)$ are tight whereas $(a, h, g, d, c)$ is not.

In Lemmas 19 to 23 we consider an embedded graph $\mathcal{G}$, a spanning tree $T$ and a subgraph $S$ in the tree-interval $\left[T^{-}, T^{+}\right]$. We consider the orientation $\mathcal{O}_{S}=\Phi(S)$ and compare edges and half-edges according to the $(\mathcal{G}, T)$-order.

Lemma 19 The fundamental cycle (resp. cocycle) of any edge in $S \cap \bar{T}$ (resp. $\bar{S} \cap T$ ) is $\mathcal{O}_{S}$-directed and tail-min (resp. head-min). 
Lemma 20 Let $e$ be a reverse edge $\left(\mathcal{O}_{S}(e) \neq \mathcal{O}_{T}(e)\right)$. Then, $e$ is in $S$ if an only if it is in a directed cycle (otherwise it is in a directed cocycle).

The (easy) proofs of Lemmas 19 and 20 are omitted.

Lemma 21 An edge e is in $S \cap \bar{T}$ (resp. $\bar{S} \cap T$ ) if and only if it is minimal in a tail-min (resp. head-min) directed cycle (resp. cocycle).

In order to prove Lemma 22 we shall use the following classical result.

Lemma 22 Let $D$ be a cocycle and let $V_{1}$ and $V_{2}$ be the connected components after deletion of $D$. If a directed cycle $C$ contains an arc oriented from $V_{1}$ to $V_{2}$ then it also contains an arc oriented from $V_{2}$ to $V_{1}$.

Proof of Lemma 21 We only prove that if an edge is minimal in a tail-min directed cycle then it is in $\in S \cap \bar{T}$. The reverse implication is given by Lemma 19. The proof of the dual equivalence ( $e$ is minimal in a tail-min directed cycle if and only if $e$ is in $\bar{S} \cap T$ ) is similar.

Let $e=\left\{h_{1}, h_{2}\right\}$ with $h_{1}<h_{2}$ be a minimal edge in a tail-min directed cycle $C$. We want to prove that $e$ is in $S \cap \bar{T}$. Observe first that $\mathcal{O}_{S}(e)=\left(h_{1}, h_{2}\right)$ (since $h_{\min }(C)=h_{1}$ and $C$ is tail-min). We now prove successively the following points.

- The edge e is not in $\bar{S} \cap T$. Otherwise, the edge $e$ would be both in a directed cycle $C$ and in a directed cocycle by Lemma 19.

- The edge $e$ is not in $S \cap T$. Suppose the contrary. Since $e$ is in $T$, the $\operatorname{arc} \mathcal{O}_{S}(e)=$ $\left(h_{1}, h_{2}\right)=\mathcal{O}_{T}(e)$ is not reverse. Let $D$ be the fundamental cocycle of $e$. Let $v_{1}$ and $v_{2}$ be the endpoints of $h_{1}$ and $h_{2}$ respectively and let $V_{2}$ be set of descendants of $v_{2}$. Recall that $v_{1}$ is the parent of $v_{2}$ in $T$ (Lemma 5 ) and that $D$ is the cocycle defined by $V_{2}$. Since the cycle $C$ is directed and the $\operatorname{arc} \mathcal{O}_{S}(e)$ in $C \cap D$ is directed toward $V_{2}$, there is an edge $e^{\prime}$ in $C \cap D$ with $\mathcal{O}_{S}\left(e^{\prime}\right)$ directed away from $V_{2}$ by Lemma 22. This situation is represented in Figure 18. Since $e$ is minimal in the cycle $C$, we have $e<e^{\prime}$. Therefore, the $\operatorname{arc} \mathcal{O}_{T}\left(e^{\prime}\right)$ is directed toward $V_{2}$ by Lemma 6 . Thus, $e^{\prime}$ is reverse. The edge $e^{\prime}$ is reverse and contained in a directed cycle, therefore it is in $S$ by Lemma 20. We have shown that $e^{\prime}$ is in $S \cap \bar{T}$. But this is impossible since $e<e^{\prime}$ is in the fundamental cycle of $e^{\prime}$.

- The edge $e$ is in $S \cap \bar{T}$. We know from the preceding points that $e$ is in $\bar{T}$. Hence, $\mathcal{O}_{T}(e)=\left(h_{2}, h_{1}\right) \neq \mathcal{O}_{S}(e)$. Thus, $e$ is reverse in a directed cycle. Therefore, $e$ is in $S$ by Lemma 20.

Lemma 23 The fundamental cycle (resp. cocycle) of any edge in $S \cap \bar{T}$ (resp. $\bar{S} \cap T$ ) is tight.

Proof. We prove that the fundamental cycle of an edge in $S \cap \bar{T}$ is tight. The proof of the dual property (concerning edges in $\bar{S} \cap T$ ) is similar. Let $e^{*}$ be in $S \cap \bar{T}$. Recall that $e^{*}=e_{\min }(C)$. By Lemma 19, the fundamental cycle $C$ of $e^{*}$ is directed. We want to prove 


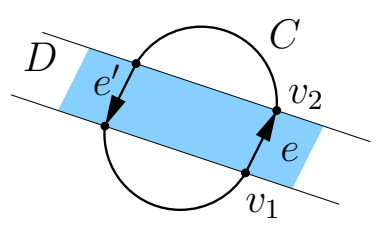

Figure 18: The directed cycle $C$, the fundamental cocycle $D$ and the edges $e$ and $e^{\prime}$.

that $C$ is tight. Suppose not and consider a directed cycle $C^{\prime}$ with $e_{\min }\left(C^{\prime}\right)=e_{\min }(C)=e^{*}$ and $e=e_{\min }\left(C \triangle C^{\prime}\right) \in C$. The edge $e$ is in the fundamental cycle $C$ of $e^{*}$, hence $e^{*}$ is in fundamental cocycle $D$ of $e$. This situation is represented in Figure 19. Let $v_{1}$ and $v_{2}$ be the endpoints of $e$ with $v_{1}$ parent of $v_{2}$ in $T$. Let $V_{2}$ be the set of descendants of $v_{2}$. Recall that $D$ is the cocycle defined by $V_{2}$. The edge $e$ is in the fundamental cycle of $e^{*}$ which is $(\mathcal{G}, T)$ active, hence $e^{*}<e$. Therefore, the $\operatorname{arc} \mathcal{O}_{T}\left(e^{*}\right)$ is directed away from $V_{2}$ by Lemma 6. Since $e^{*}$ is in $S \cap \bar{T}$, the $\operatorname{arc} \mathcal{O}_{S}\left(e^{*}\right)$ is reverse, hence is directed toward $V_{2}$. Since the cycle $C^{\prime}$ is directed and the $\operatorname{arc} \mathcal{O}\left(e^{*}\right)$ in $C^{\prime} \cap D$ is directed toward $V_{2}$, there is an $\operatorname{arc} \mathcal{O}_{S}\left(e^{\prime}\right)$ in $C^{\prime} \cap D$ oriented away from $V_{2}$ by Lemma 22. Observe that $e^{\prime}$ is not in the fundamental cycle $C$ since $C \subseteq T+e^{*}$ and $D \subseteq \bar{T}+e$. Thus, $e^{\prime}$ is in $C \triangle C^{\prime}$ and $e^{\prime}>e$. Hence, by Lemma 6 , the $\operatorname{arc} \mathcal{O}_{T}\left(e^{\prime}\right)$ in the fundamental cocycle $D$ of $e$ is directed toward $V_{2}$. Thus, the $\operatorname{arc} \mathcal{O}_{S}\left(e^{\prime}\right) \neq \mathcal{O}_{T}\left(e^{\prime}\right)$ is reverse. Since $e^{\prime}$ is reverse and contained in a directed cycle, it is in $S$ by Lemma 20. We have shown that $e^{\prime}$ is in $S \cap \bar{T}$. But this is impossible. Indeed $e^{\prime}$ is not $(\mathcal{G}, T)$-active since its fundamental cycle contains $e$ which is less than $e^{\prime}$.

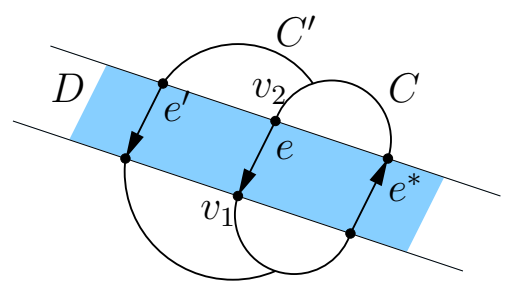

Figure 19: The directed cycles $C$ and $C^{\prime}$ and the cocycle $D$.

Proof of Proposition 18. We consider a subgraph $S_{0}$ in the tree-interval $\left[T_{0}^{-}, T_{0}^{+}\right]$ and the orientation $\mathcal{O}_{S_{0}}=\Phi\left(S_{0}\right)$. We want to prove that the procedure $\Psi$ returns the subgraph $S_{0}$. We compare edges and half-edges according to the $\left(\mathcal{G}, T_{0}\right)$-order denoted by $<$ : we say that an edge or half-edge is greater or less than another. We also compare edges and half-edges according to their order of visit during the algorithm: we say that an edge or half-edge is before or after another. We denote by $t$ the motion function associated with $T_{0}$. We denote by $h_{i}=t^{i}\left(h_{0}\right)$ the $i^{t h}$ half-edge for the $\left(\mathcal{G}, T_{0}\right)$-order. Also, for every half-edge $h$, we denote $F_{h}=\left\{e=\left\{h_{1}, h_{2}\right\}\right.$ such that $\left.\min \left(h_{1}, h_{2}\right)<h\right\}, T_{h}=T_{0} \cap F_{h}$ and $S_{h}=S_{0} \cap F_{h}$.

We want to prove that at the beginning of the $i^{\text {th }}$ core step, $h=h_{i}, F=F_{h}, T=T_{h}$, $S=S_{h}$, where $h$ is the current half-edge. We proceed by induction on the number 
of core steps. The property holds for the first $(i=0)$ core step since $h=h_{0}$ and $F_{h_{0}}=T_{h_{0}}=S_{h_{0}}=\emptyset$. Suppose the property holds for all $i \leq k$. By the induction hypothesis, the $\left(\mathcal{G}, T_{0}\right)$-order and the order of visit coincide on the edges and half-edges of $F$. In particular, if $C$ is any set not contained in $\bar{F}$, then $h_{\min }(C)=h_{\text {first }}(C)$ and $e_{\min }(C)=e_{\text {first }}(C)$. Suppose the edge $e$ containing the current half-edge $h$ is not in $F=F_{h}$. In this case, the current half-edge $h$ (resp. edge $e$ ) is less than any other halfedge (resp. edge) in $\bar{F}$. We consider the different cases $(a),(b),(c),\left(a^{\prime}\right),\left(b^{\prime}\right),\left(c^{\prime}\right)$. We will prove successively the following properties.

- Condition (a) is equivalent to $e \in S_{0} \cap \overline{T_{0}}$.

- Suppose Condition (a) holds: $h$ is a tail and $e$ is in a directed cycle $C \subseteq \bar{F}$. Since, $C \subseteq \bar{F}$, the current half-edge $h$ is minimal in $C$. Since $h$ is a tail, the directed cycle $C$ is tail-min. Thus, $e$ is in $S_{0} \cap \overline{T_{0}}$ by Lemma 21 .

- Conversely, if $e$ is in $S_{0} \cap \overline{T_{0}}$, then $e$ is minimal in a tail-min directed cycle $C$ by Lemma 21. Therefore, $h$ is a tail and $C \subseteq \bar{F}$.

- Condition ( $\left.a^{\prime}\right)$ is equivalent to $e \in \overline{S_{0}} \cap T_{0}$.

The proof is the similar to the proof of the preceding point.

- Condition (b) is equivalent to $e \in \overline{S_{0}} \cap \overline{T_{0}}$ and $\mathcal{O}_{S_{0}}(e)$ is reverse.

- Suppose Condition (b) holds: $h$ is a tail and $e$ is in a head-first directed cocycle $D \nsubseteq \bar{F}$ such that for all directed cocycle $D^{\prime}$ with $e_{\text {first }}\left(D^{\prime}\right)=e_{\text {first }}(D)$ either $e \in D^{\prime}$ or $D \triangle D^{\prime} \nsubseteq \bar{F}$ and $e_{\text {first }}\left(D \triangle D^{\prime}\right) \in D^{\prime}$. Since the $\left(\mathcal{G}, T_{0}\right)$-order and the order of visit coincide on $F$ we have $h_{\text {min }}(D)=h_{\text {first }}(D)$. Since the cocycle $D$ is head-first, it is tail-min. The edge $e^{*}:=e_{\min }(D)$ is minimal in a head-min directed cocycle, hence $e^{*}$ is in $\overline{S_{0}} \cap T_{0}$ by Lemma 21 . Let $D^{*}$ be the fundamental cocycle of $e^{*}$. Recall that $e_{\min }\left(D^{*}\right)=e^{*}=e_{\min }(D)$ We want to prove that $e$ is in $D^{*}$. Suppose $e$ is not in $D^{*}$. By Condition $(b)$, we have $D \triangle D^{*} \nsubseteq \bar{F}$ and $e_{\text {first }}\left(D \triangle D^{*}\right) \in D^{*}$. But this is impossible since $e_{\min }\left(D \triangle D^{*}\right)=e_{\text {first }}\left(D \Delta D^{*}\right)$ and $D^{*}$ is tight by Lemma 23 . Thus, $e$ is indeed in the fundamental cocycle $D^{*}$ of $e^{*}$. Since $e^{*}$ is in $\overline{S_{0}} \cap T_{0}$, the edge $e$ is in $\overline{T_{0}}$ and also in $\overline{S_{0}}$ by Lemma 13. Moreover the arc $\mathcal{O}_{S_{0}}(e)$ is reverse.

- Conversely, suppose that $e$ is in $\overline{S_{0}} \cap \overline{T_{0}}$ and that the arc $\mathcal{O}_{S_{0}}(e)$ is reverse. The current half-edge $h$ is the least half-edge of $e$. Since $e$ is external, $h$ is the head of the $\operatorname{arc} \mathcal{O}_{T_{0}}(e)$ and the tail of the reverse arc $\mathcal{O}_{S_{0}}(e)$. Since $\mathcal{O}_{S_{0}}(e)$ is reverse, the external edge $e$ is in the fundamental cocycle $D$ of an edge $e^{*} \in \overline{S_{0}} \cap T_{0}$. The cocycle $D$ is head-min, directed and tight by Lemmas 19 and 23. Since $e^{*}=e_{\min }(D)$, the edge $e^{*}$ is less than $e$. Therefore $e^{*}$ is before $e$ and $D \nsubseteq \bar{F}$. The cocycle $D$ is head-first since $h_{\text {first }}(D)=h_{\min }(D)$. Consider any directed cocycle $D^{\prime}$ such that $e_{\text {first }}\left(D^{\prime}\right)=e_{\text {first }}(D)=e^{*}$ and $e \notin D^{\prime}$. We want to prove that $D \triangle D^{\prime} \nsubseteq \bar{F}$ and $e_{\text {first }}\left(D \triangle D^{\prime}\right) \in D^{\prime}$. Since $D$ is tight, the edge $e^{\prime}=e_{\min }\left(D \triangle D^{\prime}\right)$ is in $D^{\prime}$. Since $e$ is in $D \triangle D^{\prime}$, the edge $e^{\prime}$ is less than $e$, hence it is in $F$. Therefore, $D \triangle D^{\prime} \nsubseteq \bar{F}$ and $e_{\text {first }}\left(D \triangle D^{\prime}\right)=e_{\min }\left(D \triangle D^{\prime}\right)=e^{\prime}$ is in $D^{\prime}$.

- Condition $\left(b^{\prime}\right)$ is equivalent to $e \in S_{0} \cap T_{0}$ and $\mathcal{O}_{S_{0}}(e)$ is reverse.

The proof is the similar to the proof of the preceding point. 
- Condition (c) is equivalent to $e \in S_{0} \cap T_{0}$ and is not reverse.

- Suppose Condition $(c)$ holds. In this case, Conditions $(a),\left(a^{\prime}\right),(b),\left(b^{\prime}\right)$ do not hold. Hence (by the preceding points), the edge $e$ is not in $S_{0} \triangle T_{0}$ and the arc $\mathcal{O}_{S_{0}}(e)$ is not reverse. Since $\mathcal{O}_{S_{0}}(e)$ is not reverse and the half-edge $h$ (which is the least half-edge of $e$ ) is a tail, the edge $e$ is in $T_{0}$. Since $e$ is not in $S_{0} \triangle T_{0}$, it is in $S_{0}$.

- Conversely, suppose that $e$ is in $S_{0} \cap T_{0}$ and that $\mathcal{O}_{S_{0}}(e)$ is not reverse. By the preceding points, none of the conditions $(a),\left(a^{\prime}\right),(b),\left(b^{\prime}\right)$ holds. Moreover, the half-edge $h$ (which is the least half-edge of $e$ ) is a tail.

- Condition $\left(c^{\prime}\right)$ is equivalent to $e \in \overline{S_{0}} \cap \overline{T_{0}}$ and is not reverse.

The proof is the similar to the proof of the preceding point.

By the preceding points, $e$ is added to $S$ (resp. $T$ ) in the procedure $\Psi$ if and only if $e$ is in $S_{0}$ (resp. $\left.T_{0}\right)$. Hence, the next half-edge will be $t(h)=\sigma \alpha(h)$ if $h$ is in $T_{0}$ and $\sigma(h)$ otherwise. Thus, all the properties are satisfied at the beginning of the $(k+1)^{t h}$ core step.

This concludes the proof of Theorem 16. We have also proved the following property that will be useful in the next section.

Lemma 24 During the execution of the procedure $\Psi$ on an orientation $\mathcal{O}$, the half-edges are visited in $(\mathcal{G}, T)$-order, where $T$ is the spanning tree $\Delta \circ \Psi(\mathcal{O})$.

\section{Specializations of the bijection between subgraphs and orientations}

In this section we study several restrictions of the bijection $\Phi$ between subgraphs and orientations. More precisely we shall look at the restriction of $\Phi$ to each family of subgraphs defined by combining the four criteria forest, internal, connected, external. In Figure 20 we have organized the subgraphs according to these criteria. We have also represented the orientations associated to each subgraph by the mapping $\Phi$. As Figure 20 suggests, there are nice correspondence between the properties of the subgraphs and the properties of the associated orientations. Recall that the families of subgraphs defined by combining the criteria forest, internal, connected, external are counted by the evaluations $T_{G}(i, j), 0 \leq i, j \leq 2$ of the Tutte polynomial. By studying the restriction of $\Phi$ to each of these families we shall obtain a combinatorial interpretation for each of the evaluations $T_{G}(i, j), 0 \leq i, j \leq 2$ in terms of orientations or outdegree sequences (see Theorem 41).

\subsection{Connected subgraphs and external subgraphs}

In this subsection we study the restriction of $\Phi$ to connected and to external subgraphs. 

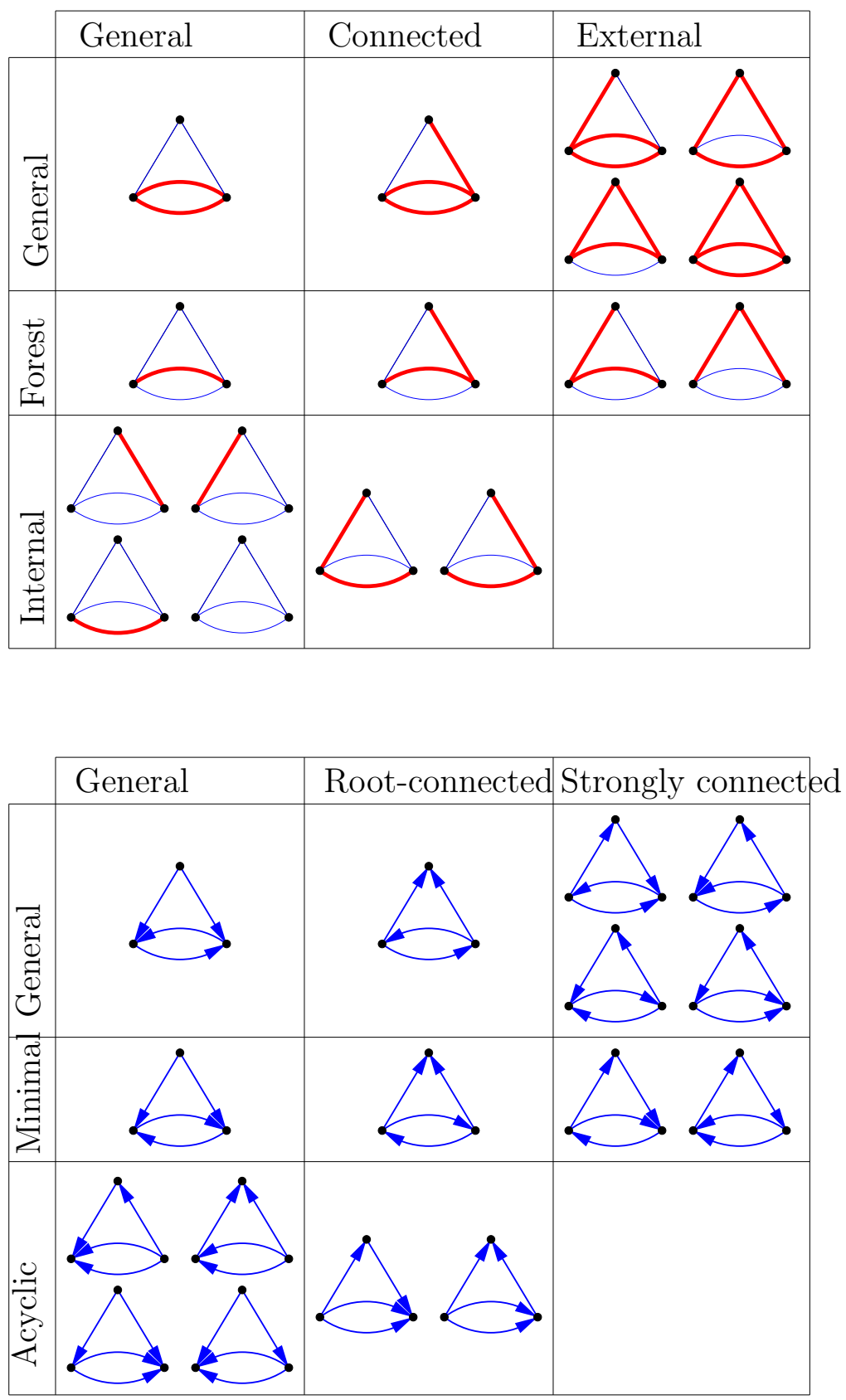

Figure 20: Subgraphs in each category defined by the four criteria forest, internal, connested, external and the corresponding orientations. The categories go from the most genaral to the most constrained from left to right and from up to down. The non-connected subgraphs (resp. non-external connected subgraphs, external subgraphs) are in column 1 (resp. 2, 3). The subgraphs that are not forests (resp. the forests that are not internal, the internal forests) are in line 1 (resp. 2, 3).

THE ELECTRONIC JOURNAL OF COMBINATORICS 15 (2008), \#R109

28 
Proposition 25 Let $\mathcal{G}$ be an embedded graph and let $v_{0}$ be the root-vertex. The orientation $\mathcal{O}_{S}$ is $v_{0}$-connected if and only if the subgraph $S$ is connected.

Lemma 26 Let $\mathcal{G}$ be an embedded graph and let $T$ be a spanning tree. Let $D$ be a cut and let $\mathcal{G}_{0}$ be the connected component of $\mathcal{G}$ containing the root-vertex $v_{0}$ after $D$ is removed. Then, the half-edge $h_{\min }(D)$ is incident to $\mathcal{G}_{0}$. Moreover, every half-edge not in $\mathcal{G}_{0}$ is greater than or equal to $h_{\min }(D)$.

Proof. Let $t$ be the motion function of $T$. If a half-edge $h$ is incident to $\mathcal{G}_{0}$ and is not in $D$ then $t(h)$ is incident to $\mathcal{G}_{0}$. Since the root $h_{0}$ is incident to $\mathcal{G}_{0}$, the half-edge $h_{\min }(D)$ is also incident to $\mathcal{G}_{0}$ and is less than any half-edge not in $\mathcal{G}_{0}$.

Lemma 27 An orientation is $v_{0}$-connected if and only if it has no head-min directed cocycle.

\section{Proof.}

- If there is a head-min directed cocycle, this cocycle is directed toward the component containing $v_{0}$ by Lemma 26. Therefore, the vertices in the other components are not reachable from $v_{0}$ and the orientation is not $v_{0}$-connected.

- If the orientation is not $v_{0}$-connected we consider the cut $D$ defined by the set $V_{0}$ of vertices reachable from $v_{0}$. The cut $D$ is directed toward $V_{0}$, hence is head-min by Lemma 26. Let $v_{1}$ be the endpoint of the edge $e=e_{\min }(D)$ that is not in $V_{0}$. Let $V_{1}$ be the set of vertices in the connected component containing $v_{1}$ after the cut $D$ is deleted. The set of edges $D_{1}$ with one endpoint in $V_{0}$ and one endpoint in $V_{1}$ is a cocycle contained in $D$. Since every edge in $D_{1}$ is directed away from $V_{0}$ the cocycle $D_{1}$ directed. Since $h_{\min }\left(D_{1}\right)=h_{\min }(D)$ is a head, the cocycle $D_{1}$ is head-min.

Proof of Proposition 25. Let $S$ be a subgraph in $\left[T^{-}, T^{+}\right]$. The orientation $\mathcal{O}_{S}$ is $v_{0}$-connected if and only if there is no head-min directed cocycle by Lemma 27 . An edge is in $\bar{S} \cap T$ if and only if it is minimal in a head-min directed cocycle by Lemma 21 . Thus, $\mathcal{O}_{S}$ is $v_{0}$-connected if and only if $\bar{S} \cap T=\emptyset$. And $\bar{S} \cap T=\emptyset$ if and only if $S$ is connected by Lemma 14 .

We now study the restriction of the bijection $\Phi$ to external subgraphs.

Proposition 28 Let $\mathcal{G}$ be an embedded graph and let $S$ be a subgraph. The orientation $\mathcal{O}_{S}$ is strongly connected if and only if $S$ is external.

Lemma 29 Let $T$ be a spanning tree and let $e$ be an edge of $T$. Let $u$ and $v$ be the endpoints of $e$ with the convention that $u$ is the parent of $v$. For any connected subgraph $S$ in $\left[T^{-}, T^{+}\right]$, the vertex $v$ is $\mathcal{O}_{s}$-reachable from its parent $u$. 
Proof. For any connected subgraph $S$ in $\left[T^{-}, T^{+}\right]$, the set $\bar{S} \cap T$ is empty by Lemma 14 . If the fundamental cocycle of the edge $e$ contains no edge of $S \cap \bar{T}$, then the arc $\mathcal{O}_{S}(e)$ is not reverse. In this case, the $\operatorname{arc} \mathcal{O}_{S}(e)=\mathcal{O}_{T}(e)$ is directed from $u$ to $v$ by Lemma 5 . Suppose now that the fundamental cocycle of $e$ contains an edge $e^{*}$ of $S \cap \bar{T}$. In this case, $e$ is in the fundamental cycle $C^{*}$ of $e^{*}$ which is $\mathcal{O}_{S}$-directed by Lemma 19. Therefore, the vertex $v$ is $\mathcal{O}_{s}$-reachable from $u$ (and vice-versa).

Lemma 30 Let $\mathcal{G}$ be an embedded graph. Let $T$ be a spanning tree and let $S$ be a connected subgraph in $\left[T^{-}, T^{+}\right]$. An edge $e$ is minimal in an $\mathcal{O}_{S^{-}}$directed cocycle if and only if $e$ is an internal $(\mathcal{G}, T)$-active edge.

Proof. Since the subgraph $S$ is connected, the subset $\bar{S} \cap T$ is empty by Lemma 14 and the orientation $\mathcal{O}_{S}$ is $v_{0}$-connected by Lemma 25 .

- Suppose that the edge $e$ is an internal $(\mathcal{G}, T)$-active edge. The edge $e$ is minimal in its fundamental cocycle $D$. We want to prove that $D$ is $\mathcal{O}_{S}$-directed. Note first that $e$ is not in $S \triangle T$ (since $e$ is in $T$ and $\bar{S} \cap T=\emptyset$ ). No other edge of $D$ is in $S \triangle T$ since none is $(\mathcal{G}, T)$-active. Hence, $\mathcal{O}_{S}(e)=\mathcal{O}_{T}(e)$. Let $e^{\prime} \neq e$ be an edge in the fundamental cocycle $D$ of $e$. The fundamental cycle of $e^{\prime}$ does not contain any edge of $\bar{S} \cap T$ since this edge is empty. Hence, $\mathcal{O}_{S}\left(e^{\prime}\right)=\mathcal{O}_{T}\left(e^{\prime}\right)$. Thus, the orientations $\mathcal{O}_{S}$ and $\mathcal{O}_{T}$ coincide on the cocycle $D$. By Lemma 10, the cocycle $D$ is $\mathcal{O}_{T}$-directed, hence it is $\mathcal{O}_{S}$-directed.

- Suppose that $e=\left\{h_{1}, h_{2}\right\}$ with $h_{1}<h_{2}$ is minimal in an $\mathcal{O}_{S}$-directed cocycle $D$. We want to prove that $e$ is an internal $(\mathcal{G}, T)$-active edge. We prove successively the following properties:

- The half-edge $h_{1}$ is a tail. Otherwise, the cocycle $D$ is head-min. (This is impossible by Lemma 27 since $\mathcal{O}_{S}$ is is $v_{0}$-connected.) - The edge $e$ is in $T$. If $e$ is not in $T$, then the arc $\mathcal{O}_{S}(e)=\left(h_{1}, h_{2}\right)$ is reverse. Thus, the fundamental cycle $C$ of $e$ contains an edge of $S \triangle T$. Since $C \subseteq T+e$ and $\bar{S} \cap T=\emptyset$, the edge $e$ is in $S \cap \bar{T}$. Thus, the cycle $C$ is $\mathcal{O}_{S}$-directed by Lemma 19. This is impossible since $e$ cannot be both is a directed cycle and a directed cocycle.

- The edge e is $(\mathcal{G}, T)$-active. Since the edge $e$ is in $T$, the $\operatorname{arc} \mathcal{O}_{S}(e)=\left(h_{1}, h_{2}\right)=\mathcal{O}_{T}(e)$ is not reverse. Let $v_{1}$ and $v_{2}$ be the endpoints of $h_{1}$ and $h_{2}$ respectively. Let $\mathcal{G}_{2}$ be the connected component of $\mathcal{G}$ containing $v_{2}$ once the cocycle $D$ is removed. The arc $\mathcal{O}_{S}(e)$ is directed toward $v_{2}$, thus the cocycle $D$ is directed toward $\mathcal{G}_{2}$. By Lemma 29, all the descendants of $v_{2}$ are reachable from $v_{2}$, hence they are all in $\mathcal{G}_{2}$. Let $e^{\prime}$ be an edge in the fundamental cocycle $D^{\prime}$ of $e$. Since one of the endpoints of $e^{\prime}$ is a descendant of $v_{2}$, the edge $e^{\prime}$ is either in $D$ or in $\mathcal{G}_{2}$. Since the minimal half-edge $h_{1}$ of $D$ is not incident to $\mathcal{G}_{2}$, every edge in $D \cup \mathcal{G}_{2}$ is greater than or equal to $e$ by Lemma 26. Thus, $e^{\prime}$ is greater than $e$. The edge $e$ is minimal in its fundamental cocycle $D$, that is, $e$ is $(\mathcal{G}, T)$-active.

Proof of Proposition 28. Let $S$ be a subgraph in $\left[T^{-}, T^{+}\right]$.

- Suppose that the subgraph $S$ is external. The subgraph $S$ is connected and there is no $(\mathcal{G}, T)$-active edge, hence there is no $\mathcal{O}_{S}$-directed cocycle by Lemma 30 . Thus, the 
orientation $\mathcal{O}_{S}$ is strongly connected.

- Suppose that the orientation $\mathcal{O}_{S}$ is strongly connected. The subgraph $S$ is connected (since $\mathcal{O}_{S}$ is $v_{0}$-connected) and there is no $\mathcal{O}_{S}$-directed cocycle, hence there is no $(\mathcal{G}, T)$ active edge by Lemma 30. Thus, the subgraph $S$ is external.

\subsection{Forests and internal forests}

In this subsection we study the restriction of the bijection $\Phi$ to forests and to internal subgraphs.

Let $\mathcal{G}$ be an embedded graph and let $\mathcal{O}$ be an orientation. We compare half-edges according to the $(\mathcal{G}, T)$-order, where $T=\Delta \circ \Psi(\mathcal{O})$. We say that the orientation $\mathcal{O}$ is minimal if there is no tail-min $\mathcal{O}$-directed cycle. We shall see (Lemma 34 ) that for any outdegree sequence $\delta$ there is a unique minimal $\delta$-orientation. Recall that for any outdegree sequence $\delta$ of a embedded planar graph, a lattice $\mathcal{L}_{\delta}$ can be defined on the set of $\delta$-orientations $[20,38]$. In the planar case, our notion of minimality coincide with the notion of being minimal in the lattice $\mathcal{L}_{\delta}$.

Proposition 31 The orientation $\mathcal{O}_{S}$ is minimal if and only if the subgraph $S$ is a forest.

Proof. Let $T=\Delta(S)$. By Lemma 21, an edge is in $S \cap \bar{T}$ if and only if it is minimal in a tail-min directed cycle. Thus, the orientation $\mathcal{O}_{S}$ is minimal if and only if $S \cap \bar{T}=\emptyset$. And $S \cap \bar{T}=\emptyset$ if and only if $S$ is a forest by Lemma 14 .

Proposition 32 The orientation $\mathcal{O}_{S}$ is acyclic if and only if the subgraph $S$ is internal.

In order to prove Proposition 32 we need to define a linear order, the postfix order, on the vertex set. For any vertex $v \neq v_{0}$ we denote by $h_{v}$ the half-edge incident to $v$ and contained in the edge linking $v$ to its parent in $T$. The postfix order, denoted by $<_{\text {post }}$, is defined by $v<_{\text {post }} v_{0}$ for $v \neq v_{0}$ and $v<_{\text {post }} v^{\prime}$ if $h_{v}<h_{v^{\prime}}$ for $v, v^{\prime} \neq v_{0}$. The postfix order is illustrated in Figure 21.

Lemma 33 Let $T$ be a spanning tree and let $e$ be an edge. The arc $\mathcal{O}_{T}(e)$ is directed toward its greatest endpoint (for the postfix order) if and only if the edge e is external $(\mathcal{G}, T)$-active.

Lemma 33 is illustrated by Figure 21.

Proof. Recall from Lemma 6 that a half-edge $h$ is incident to a descendant of $v$ if and only if $h_{v}^{\prime}<h \leq h_{v}$, where $h_{v}^{\prime}=\alpha\left(h_{v}\right)$ is the other half of the edge containing $h_{v}$.

- Consider an internal edge $e$. Let $u$ and $v$ be the endpoints of $e$ with $u$ parent of $v$. By 
Lemma 5 , the $\operatorname{arc} \mathcal{O}_{T}(e)$ is directed toward $v$. We want to prove that $v<_{\text {post }} u$. If $u=v_{0}$, the inequality holds. Else, the half-edges $h_{u}$ and $h_{v}$ exist. Moreover, the half-edge $h_{v}$ is incident to a descendant of $u$, hence $h_{v}<h_{u}$ and $v<{ }_{\text {post }} u$.

- Consider an external edge $e$. We write $e=\left\{h_{1}, h_{2}\right\}$ with $h_{1}<h_{2}$ and denote by $u$ and $v$ the endpoints of $h_{1}$ and $h_{2}$ respectively. By definition, the $\operatorname{arc} \mathcal{O}_{T}(e)$ is directed toward $u$. We want to prove that $v \leq_{\text {post }} u$ if and only if $e$ is $(\mathcal{G}, T)$-active.

- Suppose the edge $e$ is $(\mathcal{G}, T)$-active. Then, the vertex $v$ is a descendant of $u$ by Lemma 7 . The half-edge $h_{v}$ is incident to a descendant of $u$, hence $h_{v} \leq h_{u}$ and $v \leq_{\text {post }} u$.

- Suppose that $v \leq_{\text {post }} u$. If $u=v_{0}$, the vertex $v$ is a descendant of $u$ and the edge $e$ is $(\mathcal{G}, T)$-active by Lemma 7 . Else, the half-edges $h_{u}$ and $h_{v}$ exist and $h_{v} \leq h_{u}$. In this case, $\alpha\left(h_{u}\right)<h_{1}<h_{2}<h_{v} \leq h_{u}$ (indeed, $h_{2}<h_{v}$ since $h_{2}$ is incident to $v$ and $\alpha\left(h_{u}\right)<h_{1}$ since $h_{1}$ is incident to $u$ ), hence $v$ is a descendant of $u$ by Lemma 6 . Thus, the edge $e$ is $(\mathcal{G}, T)$-active by Lemma 7 .

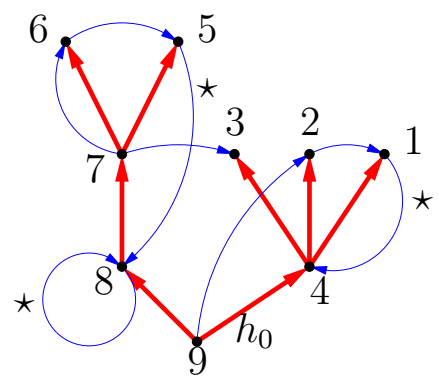

Figure 21: A spanning tree $T$, the postfix order, the orientation $\mathcal{O}_{T}$ and the external active edges (indicated by $a \star$ ).

Proof of Proposition 32. Let $S$ be a subgraph in the tree-interval $\left[T^{-}, T^{+}\right]$. We compare half-edges according to the $(\mathcal{G}, T)$-order.

- Suppose that the subgraph $S$ is internal (i.e. the tree $T$ is internal). Recall that $S \cap \bar{T}=\emptyset$. We want to prove that the orientation $\mathcal{O}_{S}$ is acyclic. Observe first that the orientation $\mathcal{O}_{T}$ is acyclic since the vertices are strictly decreasing (for the postfix order) along any $\mathcal{O}_{T}$-directed path by Lemma 33. Suppose now that there is an $\mathcal{O}_{S}$-directed cycle $C$. The $\mathcal{O}_{S}$-directed cycle $C$ contains a reverse $\operatorname{arc} \mathcal{O}(e)$ or $C$ would be $\mathcal{O}_{T}$-directed. Since $S \cap \bar{T}=\emptyset$, the reverse edges are in the fundamental cocycle of an edge of $\bar{S} \cap T$. Thus, the edge $e$ is in the fundamental cocycle $D$ of an edge of $\bar{S} \cap T$. The cocycle $D$ is directed by Lemma 19. This is impossible since $e$ cannot be both in a directed cycle and in a directed cocycle.

- Suppose that the orientation $\mathcal{O}_{S}$ is acyclic. We want to prove that the subgraph $S$ is internal (i.e. the tree $T$ is internal). Suppose there is an external $(\mathcal{G}, T)$-active edge $e$. Let $C$ be the fundamental cycle of $e$. Since $\mathcal{O}_{S}$ is minimal, we know (by Proposition 31) that $S \cap \bar{T}$ is empty. Therefore, the reverse edges are in the fundamental cocycle of an edge of $\bar{S} \cap T$. Since $e$ is active, it is not in the fundamental cocycle of an edge of $\bar{S} \cap T$. 
Since the other edges of $C$ are not active (they are less than $e$ ) they are not in $\bar{S} \cap T$. Moreover, since they are in $T$, they are not in the fundamental cocycle of an edge of $\bar{S} \cap T$. Thus, the orientations $\mathcal{O}_{S}$ and $\mathcal{O}_{T}$ coincide on the cycle $C$. By Lemma 10 , the cycle $C$ is $\mathcal{O}_{T}$-directed, hence it is $\mathcal{O}_{S}$-directed. This is impossible since $\mathcal{O}_{S}$ is acyclic.

\subsection{Minimal orientations and outdegree sequences}

In the previous subsection we proved that the bijection $\Phi$ induces a bijection between forests and minimal orientations (Proposition 31). We are now going to link minimal orientations and outdegree sequences.

Proposition 34 Let $\mathcal{G}$ be an embedded graph. For any outdegree sequence $\delta$ there exists a unique minimal $\delta$-orientation.

The rest of this subsection is devoted to the proof of Proposition 34. We first recall the link between outdegree sequences and the cycle-flips.

Consider an orientation $\mathcal{O}$ and an $\mathcal{O}$-directed cycle (resp. cocycle) $C$. Flipping the $\mathcal{O}$-directed cycle (resp. cocycle) $C$ means reversing every arc in $C$. We shall talk about cycle-flips and cocycle-flips. Observe that flipping a directed cycle does not change the outdegree sequence. Therefore, any orientation $\mathcal{O}^{\prime}$ obtained from $\mathcal{O}$ by a sequence of cycle-flips has the same outdegree sequence as $\mathcal{O}$. It was proved in [20] that the converse is also true.

Lemma 35 [20] Two orientations $\mathcal{O}$ and $\mathcal{O}^{\prime}$ have the same outdegree sequence if and only if they can be obtained from one another by a sequence of cycle-flips. Moreover, the flipped cycles can be chosen to be contained in the set $\left\{e / \mathcal{O}(e) \neq \mathcal{O}^{\prime}(e)\right\}$.

Lemma 35 is a direct consequence of the following result proved in [20].

Lemma 36 [20] Let $G$ be a graph and let $\mathcal{O}$ and $\mathcal{O}^{\prime}$ be two orientations having the same outdegree sequence. For any edge $e$ in the set $K=\left\{e^{\prime} / \mathcal{O}(e) \neq \mathcal{O}^{\prime}(e)\right\}$, there is an $\mathcal{O}$ directed cycle $C \subseteq K$ containing $e$.

Proof. (Hint) Start from the end $v$ of $\mathcal{O}(e)$ and look for an edge $e_{1}$ in $K$ directed away from $v$. This edge exists except if $v$ is also the origin of $e$ (since the number of edges directed away from $v$ is the same in $\mathcal{O}$ and $\mathcal{O}^{\prime}$ ). Repeat the process until arriving to the origin of $e$.

Recall that any very arc of an oriented graph is either in a directed cycle or a directed cocycle but not both. We say that an arc $a$ is cyclic or acyclic depending on $a$ being in a directed cycle or in a directed cocycle. We call cyclic part (resp. acyclic part) of an orientation the set of cyclic (resp. acyclic) edges. 
It is well known that the cyclic and acyclic parts are unchanged by a cycle-flip or a cocycle flip $[20,24,38]$. Indeed, it is easily seen that the cyclic part of an orientation can only grow when a directed cocycle $D$ is flipped (since no directed cycle intersects with $D)$. Since we return to the original orientation by flipping $D$ twice, we conclude that the cyclic and acyclic parts are unchanged by a cocycle-flip. Similarly, the cyclic and acyclic parts are unchanged by a cycle-flip.

We will also need the following classical result.

Lemma 37 Let $\mathcal{O}$ be an orientation and let $C$ and $C^{\prime}$ be two $\mathcal{O}$-directed cycles (resp. cocycles). Let $\mathcal{O}^{\prime}$ be the orientation obtained from $\mathcal{O}$ by flipping $C^{\prime}$. Then, the symmetric difference of $C$ and $C^{\prime}$ is a union of $\mathcal{O}^{\prime}$-directed cycles (resp. cocycles). In particular, any edge in the $\mathcal{O}$-directed cycle (resp. cocycle) $C$ is in an $\mathcal{O}^{\prime}$-directed cycle (resp. cocycle) $C^{\prime \prime} \subseteq C \cup C^{\prime}$.

Lemma 37 is illustrated by Figure 22.
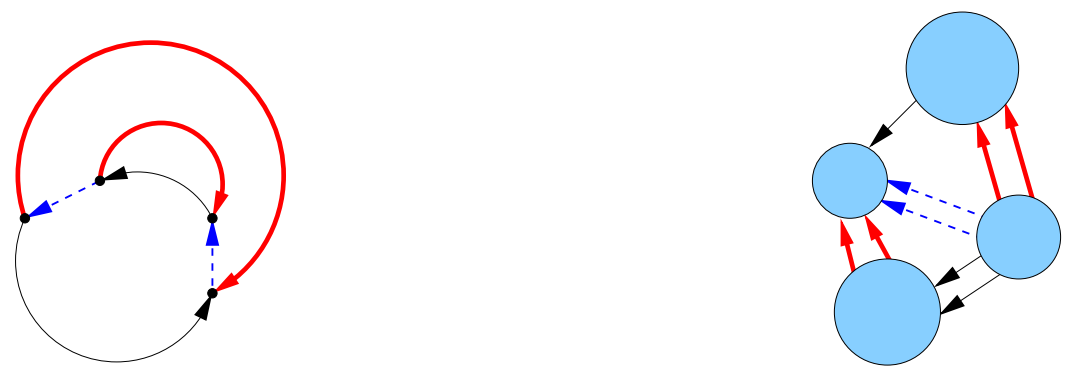

Figure 22: The $\mathcal{O}$-directed cycles (resp. cocycles) $C$ and $C^{\prime}$ (thin and thick lines) and their intersection (dashed lines).

We are now ready to prove Proposition 34. A false proof of the uniqueness of the minimal $\delta$-orientation in this proposition is as follows. If there are two different $\delta$-orientations $\mathcal{O}$ and $\mathcal{O}^{\prime}$, then these orientations differ on a directed cycle $C$. Hence, the cycle $C$ is tail-min in either $\mathcal{O}$ or $\mathcal{O}^{\prime}$. A false proof of the existence (of a minimal $\delta$-orientation) is as follows. Take any $\delta$-orientation and starts flipping cycles until no more tail-min directed cycle remains. Of course, both the uniqueness and existence proofs are false in this version since flipping a cycle changes the associated subgraph, hence the spanning tree and the order on the half-edges. However being a bit careful, one can make both proofs correct.

We consider the procedure $\Psi$ on orientations (see Definition 17). For an orientation $\mathcal{O}$ we denote by $\Psi[\mathcal{O}]$ the execution of $\Psi$ on $\mathcal{O}$. Recall (from Lemma 24) that the half-edges are visited in $(\mathcal{G}, T)$-order during $\Psi[\mathcal{O}]$, where $T$ is the spanning tree $\Delta \circ \Psi(\mathcal{O})$. Therefore, the orientation $\mathcal{O}$ is minimal if and only if Condition $(a)$ never holds during the execution $\Psi[\mathcal{O}]$. 
Lemma 38 Let $\mathcal{O}$ be an orientation. Consider the current half-edge $h$, the edge $e$ and the sets $F, S$ and $T$ at the beginning of a given core step of the execution $\Psi[\mathcal{O}]$. Let $C_{f} \subseteq \overline{F+e}$ be an $\mathcal{O}$-directed cycle and let $\mathcal{O}^{\prime}$ be the orientation obtained from $\mathcal{O}$ by flipping $C_{f}$. We want to prove that Condition (a) (resp. $(b),(c),\left(a^{\prime}\right),\left(b^{\prime}\right),\left(c^{\prime}\right)$ ) holds for the orientation $\mathcal{O}$ if and only if it holds for the orientation $\mathcal{O}^{\prime}$. (Let us insist that when evaluating the Conditions $(a), \cdots,\left(c^{\prime}\right)$ for the orientation $\mathcal{O}^{\prime}$, the symbols $F, S, T, h_{\text {first }}$ and $e_{\text {first }}$ continue to refer to the execution of $\left.\Psi[\mathcal{O}].\right)$

Proof. Note first that the orientations $\mathcal{O}$ and $\mathcal{O}^{\prime}$ coincide on the current half-edge $h$ since $e \notin C_{f}$. We now study separately the different conditions.

- Recall that $\mathcal{O}$ and $\mathcal{O}^{\prime}$ coincide on their acyclic part: the directed cocycles of $\mathcal{O}$ and $\mathcal{O}^{\prime}$ are the same. Therefore, Condition $(b)$ (resp. $\left(a^{\prime}\right)$ ) holds for $\mathcal{O}$ if and only if it holds for $\mathcal{O}^{\prime}$.

- Suppose now that Condition $(a)$ holds for $\mathcal{O}$ : the current half-edge $h$ is a tail and the edge $e$ is in an $\mathcal{O}$-directed cycle $C \subseteq \bar{F}$. By Lemma 37, the edge $e$ is also in an $\mathcal{O}^{\prime}$-directed cycle $C^{\prime} \subseteq C \cup C_{f} \subseteq \bar{F}$. Thus, Condition $(a)$ holds for $\mathcal{O}^{\prime}$. The same argument proves that if Condition $(a)$ holds for $\mathcal{O}^{\prime}$, then it holds for $\mathcal{O}\left(\mathcal{O}\right.$ is obtained from $\mathcal{O}^{\prime}$ by flipping the $\mathcal{O}^{\prime}$-directed cycle $C_{f}$ ).

- Suppose now that Condition $\left(b^{\prime}\right)$ holds for $\mathcal{O}$ : the current half-edge $h$ is a head and the edge $e$ is in a tail-first $\mathcal{O}$-directed cycle $C \nsubseteq \bar{F}$ such that for all $\mathcal{O}$-directed cycle $C^{\prime}$ with $e_{\text {first }}\left(C^{\prime}\right)=e_{\text {first }}(C)$ either $e \in C^{\prime}$ or $\left(C \triangle C^{\prime} \nsubseteq \bar{F}\right.$ and $\left.e_{\text {first }}\left(C \triangle C^{\prime}\right) \in C^{\prime}\right)$. By Lemma 37 , the edge $e^{*}=e_{\text {first }}(C)$ is in an $\mathcal{O}^{\prime}$-directed cycle $C_{1} \subseteq C \cup C_{f}$. Note that $e_{\text {first }}\left(C_{1}\right)=e^{*}$. We want to prove that Condition $\left(b^{\prime}\right)$ holds for $\mathcal{O}^{\prime}$ by considering the $\mathcal{O}^{\prime}$-directed cycle $C_{1}$. We prove successively the following properties.

- The edge $e$ is in $C_{1}$.

The edge $e^{*}$ is in the $\mathcal{O}^{\prime}$-directed cycle $C_{1}$ and not in $C_{f}$. By Lemma 37, there is an $\mathcal{O}$-directed cycle $C_{2} \subseteq C_{1} \cup C_{f}$ containing $e^{*}$ (since $\mathcal{O}$ is obtained from $\mathcal{O}^{\prime}$ by flipping $\left.C_{f}\right)$. Note that $e_{\text {first }}\left(C_{2}\right)=e^{*}$. Suppose that $e$ is not in $C_{2}$. By Condition $\left(b^{\prime}\right)$ on $C$, we have $C \triangle C_{2} \nsubseteq \bar{F}$ and $e_{\text {first }}\left(C \triangle C_{2}\right) \in C_{2}$. This is impossible since $\bar{C} \cap C_{2} \subseteq C_{f}$ (since $C_{2} \subseteq C_{1} \cup C_{f} \subseteq C \cup C_{f}$ ) and the edge $e$ in $C \cap \overline{C_{2}}$ is visited before any edge in $C_{f}$. Thus $e \in C_{2}$. Since $e \in C_{2} \subseteq C_{1} \cup C_{f}$ and $e$ is not in $C_{f}$, it is in $C_{1}$.

- For all $\mathcal{O}^{\prime}$-directed cycle $C_{1}^{\prime}$ with $e_{\text {first }}\left(C_{1}^{\prime}\right)=e_{\text {first }}\left(C_{1}\right)$ either $e \in C_{1}^{\prime}$ or $\left(C_{1} \triangle C_{1}^{\prime} \nsubseteq \bar{F}\right.$ and $\left.e_{\text {first }}\left(C_{1} \triangle C_{1}^{\prime}\right) \in C_{1}^{\prime}\right)$. (This proves that Condition $\left(b^{\prime}\right)$ is satisfied for $\left.\mathcal{O}^{\prime}\right)$.

Let $C_{1}^{\prime}$ be an $\mathcal{O}^{\prime}$-directed cycle not containing $e$ and such that $e_{\text {first }}\left(C_{1}^{\prime}\right)=e_{\text {first }}\left(C_{1}\right)=$ $e^{*}$. We want to prove that $C_{1} \triangle C_{1}^{\prime} \subseteq \bar{F}$ and $e_{\text {first }}\left(C_{1} \triangle C_{1}^{\prime}\right) \in C_{1}^{\prime}$. The edge $e^{*}$ is in the $\mathcal{O}^{\prime}$-directed cycle $C_{1}^{\prime}$ but not in $C_{f}$. By Lemma 37 , there exists an $\mathcal{O}$-directed cycle $C^{\prime} \subseteq C_{1}^{\prime} \cup C_{f}$ containing $e^{*}$. Note that $e_{\text {first }}\left(C^{\prime}\right)=e^{*}$ and that $e \notin C^{\prime}$ (since $e$ is not in $C_{f}$ nor in $C_{1}^{\prime}$ by hypothesis). By Condition $\left(b^{\prime}\right)$ on $C$, we have $C \triangle C^{\prime} \nsubseteq \bar{F}$ and $e^{\Delta}=e_{\text {first }}\left(C \triangle C^{\prime}\right) \in C^{\prime}$. We now prove the following properties.

- The edge $e^{\Delta}$ is in $\overline{C_{1}} \cap C_{1}^{\prime}$.

The edge $e^{\Delta}$ is in $C_{1}^{\prime}$ since $e^{\Delta} \notin C_{f}$ and $e^{\Delta} \in C^{\prime} \subseteq C_{1}^{\prime} \cup C_{f}$. Moreover, $e^{\Delta}$ is not in 
$C_{1}$ since $e^{\Delta} \notin C, e^{\Delta} \notin C_{f}$ and $C_{1} \subseteq C \cup C_{f}$. Thus, $e^{\Delta}$ is in $\overline{C_{1}} \cap C_{1}^{\prime}$.

- Any edge in $C_{1} \cap \overline{C_{1}^{\prime}}$ is visited after $e^{\Delta}$ during the execution $\Psi[\mathcal{O}]$.

Let $e^{\prime}$ be an edge in $C_{1} \cap \overline{C_{1}^{\prime}}$. If $e^{\prime}$ is in $C_{f}$, it is visited after $e^{\Delta}$. Else, $e^{\prime}$ is in $C$ since $e^{\prime} \in C_{1}, e^{\prime} \notin C_{f}$ and $C_{1} \subseteq C \cup C_{f}$. Moreover, $e^{\prime}$ is not in $C^{\prime}$ since $e^{\prime} \notin C_{1}^{\prime}, e^{\prime} \notin C_{f}$ and $C^{\prime} \subseteq C_{1} \cup C_{f}$. Since $e^{\prime} \in C \triangle C^{\prime}$, the edge $e^{\prime}$ is visited after $e^{\Delta}=e_{\text {first }}\left(C \triangle C^{\prime}\right)$ during the execution $\Psi[\mathcal{O}]$.

Since $e^{\Delta}$ is in $\overline{C_{1}} \cap C_{1}^{\prime}$ and any edge in $C_{1} \cap \overline{C_{1}^{\prime}}$ is visited after $e^{\Delta}$, the edge $e_{\text {first }}\left(C_{1} \triangle\right.$ $\left.C_{1}^{\prime}\right)$ is in $C_{1}^{\prime}$. Thus, Condition $\left(b^{\prime}\right)$ holds for $\mathcal{O}^{\prime}$.

We have proved that if Condition $\left(b^{\prime}\right)$ holds for $\mathcal{O}$, then it holds for $\mathcal{O}^{\prime}$. The same argument proves that if Condition $\left(b^{\prime}\right)$ holds for $\mathcal{O}^{\prime}$, then it holds for $\mathcal{O}$.

- Condition $(c)$ holds for $\mathcal{O}$ if $h$ is a tail and Conditions $(a)$ and $(b)$ do not hold for $\mathcal{O}$ By the preceding points this is true if and only if $h$ is a tail and Conditions $(a)$ and $(b)$ do not hold for $\mathcal{O}^{\prime}$. Therefore, Condition $(c)$ holds for $\mathcal{O}$ if and only if it holds for $\mathcal{O}^{\prime}$. Similarly, Condition $\left(c^{\prime}\right)$ holds for $\mathcal{O}$ if and only if it holds for $\mathcal{O}^{\prime}$.

Lemma 39 Let $\mathcal{O}$ and $\mathcal{O}^{\prime}$ be two orientations having the same outdegree sequence. For all $0 \leq i<|H|$, let $h_{i}, F_{i}, T_{i}$ and $S_{i}$ (resp. $h_{i}^{\prime}, F_{i}^{\prime}, T_{i}^{\prime}$ and $S_{i}^{\prime}$ ) be respectively the current half-edge and the sets $F, T$ and $S$ at the beginning of the $i^{\text {th }}$ core step of the execution $\Psi[\mathcal{O}]$ (resp. $\Psi\left[\mathcal{O}^{\prime}\right]$ ). If the orientations $\mathcal{O}$ and $\mathcal{O}^{\prime}$ coincide on $h_{i}$ for all $i<k$ (that $i s, \mathcal{O}\left(e_{i}\right)=\mathcal{O}^{\prime}\left(e_{i}\right)$ where $e_{i}$ is the edge containing $\left.h_{i}\right)$, then the $k$ first core steps of the executions $\Psi[\mathcal{O}]$ and $\Psi\left[\mathcal{O}^{\prime}\right]$ are the same. In particular, $h_{i}=h_{i}^{\prime}, F_{i}=F_{i}^{\prime}, S_{i}=S_{i}^{\prime}$, and $T_{i}=T_{i}^{\prime}$ for all $i \leq k$.

Proof. We proceed by induction on $k$. Recall from Lemma 35 that the orientation $\mathcal{O}^{\prime}$ can be obtained from $\mathcal{O}$ by a sequence of cycle-flips such that the flipped cycles are contained in the set $K=\left\{e / \mathcal{O}(e) \neq \mathcal{O}^{\prime}(e)\right\}$. For $k=0$ the property obviously holds. Now suppose that the property holds for $k$ and suppose that $\mathcal{O}$ and $\mathcal{O}^{\prime}$ coincide on $h_{i}, i<k+1$. By the induction hypothesis the current half-edge $h_{k}=h_{k}^{\prime}$ and the sets $F=F_{k}=F_{k}^{\prime}$, $S=S_{k}=S_{k}^{\prime}$, and $T=T_{k}=T_{k}^{\prime}$ are the same at the beginning of the $(k+1)^{t h}$ core step of the procedures $\Psi[\mathcal{O}]$ and $\Psi\left[\mathcal{O}^{\prime}\right]$. Moreover, the set $K=\left\{e^{\prime} / \mathcal{O}\left(e^{\prime}\right) \neq \mathcal{O}^{\prime}\left(e^{\prime}\right)\right\}$ of reverse edges is contained in $\overline{F+e}$. Since $\mathcal{O}^{\prime}$ is obtained from $\mathcal{O}$ by a sequence of flips of cycles contained in $\overline{F+e}$, we know by induction on Lemma 38 that Condition $(a)$ (resp. (b), $\left.(c),\left(a^{\prime}\right),\left(b^{\prime}\right),\left(c^{\prime}\right)\right)$ holds for the orientation $\mathcal{O}$ if and only if it holds for the orientation $\mathcal{O}^{\prime}$. Therefore, the $(k+1)^{t h}$ core step is the same for the two executions $\Psi[\mathcal{O}]$ and $\Psi\left[\mathcal{O}^{\prime}\right]$. In particular, the sets $F, S$, and $T$ are modified in the same way in both executions and $h_{k+1}=h_{k+1}^{\prime}$. Thus, the property holds by induction.

Proof of Proposition 34. Recall that an orientation $\mathcal{O}$ is minimal if and only if Condition $(a)$ never holds during the execution $\Psi[\mathcal{O}]$. Thus, we need to prove that for any outdegree sequence $\delta$ there exists a unique $\delta$-orientation $\mathcal{O}$ such that Condition $(a)$ never holds during the execution $\Psi[\mathcal{O}]$. 
- Uniqueness: Let $\mathcal{O}$ and $\mathcal{O}^{\prime}$ be two (distinct) orientations having the same outdegree sequence. We take the same notations $h_{i}, F_{i}, T_{i}, S_{i}, h_{i}^{\prime}, F_{i}^{\prime}, T_{i}^{\prime}, S_{i}^{\prime}$ as in Lemma 39. Let $k$ be the first index such that $\mathcal{O}$ and $\mathcal{O}^{\prime}$ differ on $h_{k}$. By Lemma 39, we have $h_{k}=h_{k}^{\prime}$ and $F_{k}=F_{k}^{\prime}, T_{k}=T_{k}^{\prime}, S_{k}=S_{k}^{\prime}$. We can suppose without loss of generality that $h_{k}$ is a tail in $\mathcal{O}$ and a head in $\mathcal{O}^{\prime}$. We now prove that Condition $(a)$ holds for $\mathcal{O}$. By hypothesis, the edge $e$ containing $h$ is such that $\mathcal{O}(e) \neq \mathcal{O}^{\prime}(e)$. Hence, by Lemma 36, the edge $e$ is contained in an $\mathcal{O}$-directed cycle $C \subseteq K=\left\{e / \mathcal{O}(e) \neq \mathcal{O}^{\prime}(e)\right\}$. Since $\mathcal{O}$ and $\mathcal{O}^{\prime}$ coincide on $h_{i}$ for $i<k$, the set $K$ is contained in $\overline{F_{i}}$. Since $C \subseteq \overline{F_{i}}$ is $\mathcal{O}$-directed, Condition $(a)$ holds for $\mathcal{O}$.

- Existence: Let $\delta$ be an outdegree sequence. We want to find a $\delta$-orientation $\mathcal{O}$ such that Condition $(a)$ never holds during the execution $\Psi[\mathcal{O}]$. Let $\mathcal{O}_{0}$ be any $\delta$-orientation. We are going to define a set of $\delta$-orientations $\mathcal{O}_{0}, \mathcal{O}_{1}, \ldots, \mathcal{O}_{|H|}$ such that Condition $(a)$ is not satisfied during the $i$ first core steps of the execution $\Psi\left[\mathcal{O}_{i}\right]$. We prove that $\mathcal{O}_{k}$ exists by induction on $k$. Suppose the $\delta$-orientation $\mathcal{O}_{k-1}$ exists. We consider the current half-edge $h$, the edge $e$ and the sets $F, S$ and $T$ at the beginning of the $k^{t h}$ core step of $\Psi\left[\mathcal{O}_{k-1}\right]$. If either $e \in F$ or Condition $(a)$ does not hold, we define $\mathcal{O}_{k}=\mathcal{O}_{k-1}$. Else, the current half-edge $h_{k}$ is a tail (for the orientation $\mathcal{O}_{k-1}$ ) and there is an $\mathcal{O}_{k}$-directed cycle $C \subseteq \bar{F}$ containing $e$. In this case, we define $\mathcal{O}_{k}$ to be the orientation obtained from $\mathcal{O}_{k-1}$ by flipping the cycle $C$. Observe that $\mathcal{O}_{k}$ is a $\delta$-orientation in which $h_{k}$ is a head. Moreover, since $C \subseteq \bar{F}$ the two orientations $\mathcal{O}_{k-1}$ and $\mathcal{O}_{k}$ coincide on the half-edges $h_{i}$ for $i<k$, where $h_{i}$ is the current half-edge at the beginning of the $i^{t h}$ core step of the execution $\Psi\left[\mathcal{O}_{k-1}\right]$. Thus, by Lemma 39 , the $k$ first core steps of the executions $\Psi\left[\mathcal{O}_{k-1}\right]$ and $\Psi\left[\mathcal{O}_{k}\right]$ are the same. Moreover, the current half-edge $h=h_{k}$ at the beginning of the $k^{t h}$ core step of $\Psi\left[\mathcal{O}_{k}\right]$ is a head (for the orientation $\mathcal{O}_{k}$ ). Hence, Condition $(a)$ does not hold at this core step. Thus, $\mathcal{O}_{k}$ is a $\delta$-orientation such that Condition $(a)$ does not hold during the $k^{\text {th }}$ first core steps of the execution $\Psi\left[\mathcal{O}_{k}\right]$. The orientations $\mathcal{O}_{0}, \mathcal{O}_{1}, \ldots, \mathcal{O}_{|H|}$ exist by induction. In particular, the $\delta$-orientation $\mathcal{O}_{|H|}$ is such that Condition $(a)$ never holds during the execution $\Psi\left[\mathcal{O}_{|H|}\right]$.

From Proposition 31 and 34 one obtains the following bijection between outdegree sequences and forests.

Proposition 40 Let $\mathcal{G}$ be an embedded graph. The mapping $\Gamma$ which associates with any subgraph $S$ the outdegree sequence of the orientation $\mathcal{O}_{S}$ establishes a bijection between the forests and the outdegree sequences of $\mathcal{G}$.

Another bijection between outdegree sequences and forests was established in [29] after Stanley asked for such a bijection [41].

\subsection{Summary of the specializations and further refinements}

From Propositions 25, 28, 31 and 32 we can characterize the orientations associated with each class of subgraphs defined by the criteria forest, internal, connected, external. Each 
class of subgraphs is counted by a specialization of the Tutte polynomial given in Table 14 . Our results are summarized in the following theorem.

Theorem 41 Let $\mathcal{G}$ be an embedded graph and let $v_{0}$ be the root-vertex.

1. The $v_{0}$-connected orientations are in bijection with the connected subgraphs counted by $T_{G}(1,2)$.

2. The strongly connected orientations are in bijection with the external subgraphs counted by $T_{G}(0,2)$.

3. The outdegree sequences are in bijection with minimal orientations, which are in bijection with forests counted by $T_{G}(2,1)$.

4. The acyclic orientations are in bijection with internal forests counted by $T_{G}(2,0)$.

5. The $v_{0}$-connected outdegree sequences are in bijection with $v_{0}$-connected minimal orientations which are in bijection with spanning trees counted by $T_{G}(1,1)$.

6. The strongly connected outdegree sequences are in bijection with strongly connected minimal orientations which are in bijection with external spanning trees counted by $T_{G}(0,1)$.

7. The $v_{0}$-connected acyclic orientations are in bijection with internal spanning trees counted by $T_{G}(1,0)$.

The enumerative corollaries of these bijections are not new and precise references were given in the introduction. We will now consider some refinements of Theorem 41. For instance, we have proved that the acyclic orientations of a graph $G$ are counted by $T_{G}(2,0)$. This is the sum of the coefficients of the polynomial $T_{G}(1+x, 0)$ (which is closely related to the chromatic polynomial of $G)$. We denote by $\left[x^{i}\right] P(x)$ the coefficient of $x^{i}$ in a polynomial $P(x)$. The identities

$$
\sum_{i \in \mathbb{N}}\left[x^{i}\right] T_{G}(1+x, 0)=T_{G}(2,0)=\mid\{\text { acyclic orientations }\} \mid
$$

and

$$
\sum_{i \in \mathbb{N}}\left[x^{i}\right] T_{G}(x, 0)=T_{G}(1,0)=\mid\left\{v_{0} \text {-connected acyclic orientations }\right\} \mid,
$$

make it appealing to look for a partition of the acyclic orientations (resp. root-connected acyclic orientations) in parts of size $\left[x^{i}\right] T_{G}(1+x, 0)$ (resp. $\left.\left[x^{i}\right] T_{G}(x, 0)\right)$. Such partitions were defined by Lass in [33] using set functions algebra. The partition defined by Lass is linked to former constructions by Cartier, Foata, Gessel, Stanley and Viennot (see references in [33]). More generally, one can try to interpret the coefficients of $T_{G}(x, 1)$, $T_{G}(1+x, 1), T_{G}(x, 2), T_{G}(1+x, 2)$ etc. in terms of orientations in order to interpolate between the different specializations $T_{G}(i, j), 0 \leq i, j \leq 2$. Observe that the coefficients 
of each of these polynomials can be given an interpretation in terms of subgraphs. For instance, $\left[x^{i}\right] T_{G}(1+x, 0)$ counts internal forests with $i+1$ trees (by Theorem 11 and Lemma 14) and $\left[x^{i}\right] T_{G}(x, 0)$ counts internal spanning trees with $i$ internal embeddingactive edges (by Theorem 4).

We will now give an interpretation of the coefficients $\left[x^{i}\right] T_{G}(1+x, j)$ for $i \geq 0$ and $j=0,1,2$ in terms of orientations. Let $\mathcal{O}$ be an orientation. We define the partition of the vertex set $V$ into root-components $V=\biguplus_{0 \leq i \leq k} V_{i}$ as follows. The first root-component $V_{0}$ is the set of vertices reachable from the root-vertex $v_{0}$. If $W_{k}=\cup_{0 \leq i \leq k} V_{i} \subsetneq V$, we consider the minimal edge $e_{k}$ with one vertex in $W_{k}$ and one vertex $v_{k}$ in $\overline{W_{k}}$ (the edges are compared according to the $(\mathcal{G}, T)$-order, where $T=\Delta(\Psi(\mathcal{O})))$. Then, the $(k+1)^{t h}$ root-component is the set of vertices in $\overline{W_{k}}$ that are reachable from $v_{k}$. For instance, the root-components have been indicated for the orientation in Figure 23 (left). It is clear that $v_{0}$-connected orientations have only one root-component. Given a $v_{0}$-connected orientation $\mathcal{O}$, we define the partition of the vertex set $V$ into root-strong-components $V=\biguplus_{0 \leq i \leq k} U_{i}$ as follows. The first root-strong-component $U_{0}$ is the set of vertices that can reach the root-vertex $v_{0}$. If $W_{k}=\cup_{0 \leq i \leq k} U_{i} \subsetneq V$, we consider the minimal edge $e_{k}$ with one vertex in $W_{k}$ and one vertex $v_{k}$ in $\overline{\overline{W_{k}}}$. Then, the $(k+1)^{t h}$ root-strong-component is the set of vertices in $\overline{W_{k}}$ that can reach $v_{k}$. For instance, the root-strong-components have been indicated for the $v_{0}$-connected orientation in Figure 23 (right).
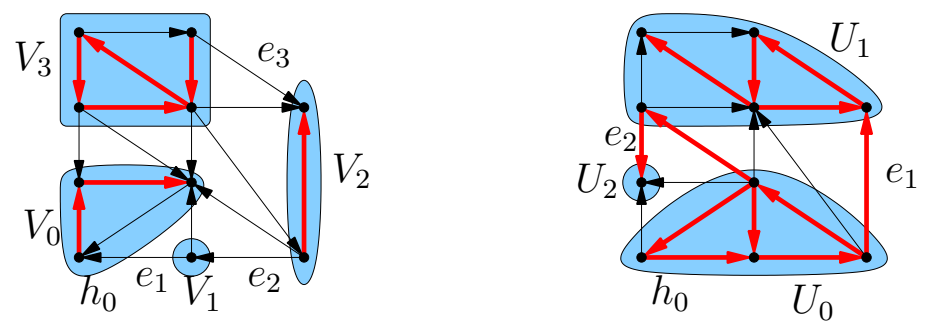

Figure 23: Left: root-components of an orientation. Right: root-strong-components of a $v_{0}$-connected orientation. The thick edges correspond to the subgraph associated with the orientation by the bijection $\Psi$.

Theorem 42 Let $\mathcal{G}$ be an embedded graph and let $v_{0}$ be the root-vertex. The coefficient $\left[x^{i}\right] T_{G}(1+x, 2)$ (resp. $\left[x^{i}\right] T_{G}(1+x, 1),\left[x^{i}\right] T_{G}(1+x, 0)$ ) counts orientations (resp. minimal orientations, acyclic orientations) with $i+1$ (non-empty) root-components. The coefficient $\left[x^{i}\right] T_{G}(x, 2)$ (resp. $\left.\left[x^{i}\right] T_{G}(x, 1),\left[x^{i}\right] T_{G}(x, 0)\right)$ counts $v_{0}$-connected orientations (resp. minimal $v_{0}$-connected orientations, acyclic $v_{0}$-connected orientations) with $i+1$ (non-empty) root-strong-components.

As mentioned above, the coefficients $\left[x^{i}\right] T_{G}(1+x, 0)$ and $\left[x^{i}\right] T_{G}(x, 0)$ had already been interpreted by Lass in [33]. The proof of Theorem 42 uses the following lemma. 
Lemma 43 Let $\mathcal{G}$ be an embedded graph and let $\mathcal{O}$ be an orientation (resp. $v_{0}$-connected orientation). We consider the spanning tree $T=\Delta(\Psi(\mathcal{O}))$ and compare the half-edges and edges according to the $(\mathcal{G}, T)$-order. Let $V_{0}, \ldots, V_{k}$ be the root-components (resp. root-strong-components). Let $e_{j}, j=1 \ldots k$ be the minimal edge in the cut defined by $W_{j}=\cup_{0 \leq i \leq j} V_{i}$. Then, $\left\{e_{1}, \ldots, e_{k}\right\}$ is the set of edges which are minimal in a head-min directed cocycle (resp. a directed cycle).

The proof of Lemma 43 is omitted.

\section{Proof of Theorem 42.}

- We first prove that the coefficient $\left[x^{i}\right] T_{G}(1+x, 2)$ (resp. $\left.\left[x^{i}\right] T_{G}(1+x, 1),\left[x^{i}\right] T_{G}(1+x, 0)\right)$ counts orientations (resp. minimal orientations, acyclic orientations) with $i+1$ rootcomponents. Let $T$ be a spanning tree with $\mathcal{I}(T)$ internal and $\mathcal{E}(T)$ external $(\mathcal{G}, T)$-active edges. By Lemma 14, the coefficient $\left[x^{i}\right](1+x)^{\mathcal{I}(T)} 2^{\mathcal{E}(T)}$ counts the subgraphs $S$ in the tree-interval $\left[T^{-}, T^{+}\right]$having $i$ edges in $\bar{S} \cap T$. Given that the tree-intervals form a partition of the set of subgraphs, the coefficient $\left[x^{i}\right] \sum_{T \text { spanning tree }}(1+x)^{\mathcal{I}(T)} 2^{\mathcal{E}(T)}$ counts the subgraphs $S$ having $i$ edges in $\bar{S} \cap \Delta(S)$. Moreover, by the characterization (4) of the Tutte polynomial, the sum $\sum_{T}(1+x)^{\mathcal{I}(T)} 2^{\mathcal{E}(T)}$ is equal to $T_{G}(1+x, 2)$. Similarly, the coefficient $\left[x^{i}\right] T_{G}(1+x, 1)$ (resp. $\left.\left[x^{i}\right] T_{G}(1+x, 0)\right)$ counts the forests (resp. internal forests) $S$ having $i$ edges in $\bar{S} \cap \Delta(S)$. By Theorem 41 and Lemma 21, the coefficient $\left[x^{i}\right] T_{G}(1+x, 2)$ (resp. $\left.\left[x^{i}\right] T_{G}(1+x, 1),\left[x^{i}\right] T_{G}(1+x, 0)\right)$ counts the orientations (resp. minimal orientations, acyclic orientations) having exactly $i$ edges which are minimal in some head-min directed cocycle. Moreover, by Lemma 43, an orientation has $i$ edges which are minimal in some head-min directed cocycle if and only if it has $i+1$ root-components.

- The proof that the coefficient $\left[x^{i}\right] T_{G}(x, 2)$ (resp. $\left[x^{i}\right] T_{G}(x, 1),\left[x^{i}\right] T_{G}(x, 0)$ ) counts $v_{0}$ connected orientations (resp. minimal $v_{0}$-connected orientations, acyclic $v_{0}$-connected orientations) with $i+1$ root-strong-components is similar.

One interesting specialization of Theorem 42 concerns bipolar orientations. Recall that, given two vertices $u$ and $v$, a $(u, v)$-bipolar orientation is an acyclic orientation such that $u$ is the unique source and $v$ is the unique sink. Bipolar orientations are well-studied structures because of their many applications in graph drawing algorithms [16]. It was shown in [28] that the coefficient $\left[x^{1}\right] T_{G}(x, 0)$, known as Crapo's $\beta$ invariant [13], counts the $(u, v)$-bipolar orientations of $G$ for any fixed pair of adjacent vertices $(u, v)$. The following proposition, illustrated in Figure 24, gives a bijective proof of this property.

Proposition 44 Let $\mathcal{G}$ be an embedded graph, let $v_{0}$ be the root-vertex and let $v_{1}$ be the other endpoint of the root-edge. The mapping $\Phi$ induces a bijection between the spanning trees having embedding-activities $(\mathcal{I}(T), \mathcal{E}(T))=(1,0)$ (counted by $\left[x^{1}\right] T_{G}(x, 0)$ ) and the $\left(v_{0}, v_{1}\right)$-bipolar orientations.

The counterpart of Proposition 44 for the notion of activities defined by Tutte in [43] was proved by Gioan and Las Vergnas in [25]. They subsequently used this bijection as 


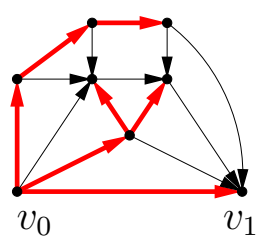

Figure 24: $\mathrm{A}\left(v_{0}, v_{1}\right)$-bipolar orientation and the corresponding spanning tree (indicated by thick lines).

the main building block in order to define a general correspondence between spanning trees and orientations. Their correspondence explains the link between the activities (of spanning trees) defined by Tutte in [43] and the activities of orientations defined by Las Vergnas in [32].

Proof. Observe first that an acyclic orientation $\mathcal{O}$ is $\left(v_{0}, v_{1}\right)$-bipolar if and only if any vertex is reachable from $v_{0}$ and can reach $v_{1}$. By Theorem 42 the coefficient $\left[x^{1}\right] T_{G}(x, 0)$ counts acyclic $v_{0}$-connected orientation having 2 root-strong-components. No vertex $v \neq v_{0}$ can reach $v_{0}$ in an acyclic $v_{0}$-connected orientation (there would be a directed path from $v_{0}$ to $v$ and back). Hence the first root-component $U_{0}$ of an acyclic $v_{0}$-connected orientation is reduced to $\left\{v_{0}\right\}$. The minimal edge with one endpoint in $U_{0}=\left\{v_{0}\right\}$ and one endpoint outside $U_{0}$ is the root-edge. Hence an acyclic $v_{0}$-connected orientation has 2 root-strong-components if and only if every vertex can reach $v_{1}$. Thus, the coefficient $\left[x^{1}\right] T_{G}(x, 0)$ counts $\left(v_{0}, v_{1}\right)$-bipolar orientations.

\section{$7 \quad$ A bijection between spanning trees and recurrent sandpile configurations}

In Section 3, we defined a mapping $\Lambda: T \mapsto \mathcal{S}_{T}$ from spanning trees to sandpile configurations. Recall from Definition 9 that the number of grains $\mathcal{S}_{T}(v)$ on the vertex $v$ in the configuration $\mathcal{S}_{T}=\Lambda(T)$ is the number of tails plus the number of external $(\mathcal{G}, T)$-active heads incident to $v$ in the orientation $\mathcal{O}_{T}=\Phi(T)$. In this section, we prove that the mapping $\Lambda$ is a bijection between spanning trees and recurrent sandpile configurations.

Theorem 45 Let $\mathcal{G}$ be an embedded graph. The mapping $\Lambda: T \mapsto \mathcal{S}_{T}$ is a bijection between the spanning trees and the recurrent sandpile configurations of $\mathcal{G}$.

Let $G=(V, E)$ be the graph underlying the embedding $\mathcal{G}$. Observe that the level of the configuration $\mathcal{S}_{T}$, that is, $\sum_{v \in V} \mathcal{S}_{T}(v)-|E|$, is the number of external $(\mathcal{G}, T)$-active edges. Indeed, every edge of $G$ has contribution 1 to the sum $\sum_{v} \mathcal{S}_{T}(v)$ except the external $(\mathcal{G}, T)$-active edges which have contribution 2 . 
Corollary 46 Let $\mathcal{G}$ be an embedded graph. The number of recurrent sandpile configurations at level $i$ is the number $\left[y^{i}\right] T_{G}(1, y)$ of spanning trees having $i$ external $(\mathcal{G}, T)$-active edges.

As mentioned above, Corollary 46 is not new. It was first proved recursively in [34] and then bijectively in [10] (using Tutte's notion of activity [43]). The Theorem 45 and Corollary 46 are illustrated by Figure 25 .
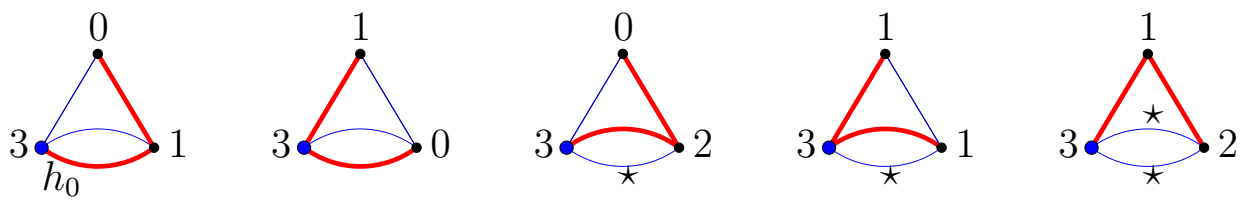

Figure 25: The spanning trees (thick lines) and the corresponding sandpile configurations. The external active edges are indicated by a $\star$.

We first prove that the image of any spanning tree is a recurrent sandpile configuration.

Proposition 47 Let $\mathcal{G}$ be an embedded graph. For any spanning tree $T$, the sandpile configuration $\mathcal{S}_{T}=\Lambda(T)$ is recurrent.

Proof. Let $v_{0}$ be the root-vertex. We consider the orientation $\mathcal{O}_{T}$ and prove successively the following properties.

- The sandpile configuration $\mathcal{S}_{T}$ is stable. Let $v$ be any vertex distinct from $v_{0}$. We want to prove that $\mathcal{S}_{T}(v)<\operatorname{deg}(v)$. Observe that any half-edge incident to $v$ has contribution at most one to $\mathcal{S}_{T}(v)$. Moreover, the half-edge $h_{v}$ incident to $v$ and contained in the edge of $T$ linking $v$ to its parent is a head by Lemma 5 . Thus, $h_{v}$ has no contribution to $\mathcal{S}_{T}(v)$, and $\mathcal{S}_{T}(v) \leq \operatorname{deg}(v)-1$.

- $\mathcal{S}_{T}\left(v_{0}\right)=\operatorname{deg}\left(v_{0}\right)$. We must prove that every half-edge incident to $v_{0}$ has contribution 1 to $\mathcal{S}_{T}\left(v_{0}\right)$. By Lemma 5 , the internal edges are oriented from parent to child in $\mathcal{O}_{T}$. Therefore any internal half-edge incident to $v_{0}$ is a tail, hence has contribution 1 to $\mathcal{S}_{T}\left(v_{0}\right)$. Let $h$ be an external half-edge incident to $v_{0}$. By definition, if the half-edge $h$ is greater than the half-edge $h^{\prime}=\alpha(h)$, then $h$ is a tail. Else, the edge $e=\left\{h, h^{\prime}\right\}$ is $(\mathcal{G}, T)$-active by Lemma 7 (since the endpoint $v_{0}$ of $h$ is an ancestor of the endpoint of $h^{\prime}$ ). Thus, any external half-edge incident to $v_{0}$ has contribution 1 to $\mathcal{S}_{T}\left(v_{0}\right)$.

- The sandpile configuration $\mathcal{S}_{T}$ is recurrent. We want to prove that there is a labeling of the vertices $v_{0}, v_{1}, \ldots, v_{|V|-1}$ such that the sequence of topplings $\mathcal{S}_{T_{-\rightarrow}}^{v_{0}} \mathcal{S}_{T \rightarrow-}^{1} v_{1} \ldots{ }^{v_{|V|-1}} \mathcal{S}_{T}^{|V|}$ is valid. Observe that in this case the configuration $\mathcal{S}_{T}$ is recurrent. Indeed, the final configuration $\mathcal{S}_{T}^{|V|}$ is equal to $\mathcal{S}_{T}$ since every vertex $v$ has been toppled once, hence has sent and received exactly $\operatorname{deg}(v, *)$ grains during the sequence of topplings (recall that $\operatorname{deg}(v, *)$ is the number of non-loop edges incident to $v$ ). In Section 6, we defined a linear order, the postfix order, on the vertex set $V$ (see Lemma 33). The root-vertex $v_{0}$ is the maximal element for this order. We want to prove that taking the unique labeling such that $v_{0}>$ 
$v_{1}>\cdots>v_{|V|-1}$ for the postfix order, the sequence of topplings $\mathcal{S}_{T}{ }_{-\rightarrow}^{v_{0}} \mathcal{S}_{T \rightarrow-\rightarrow}^{1} v_{1} \ldots v_{-\rightarrow}^{v_{|V|-1}} \mathcal{S}_{T}^{|V|}$ is valid. From the preceding point, the toppling of $v_{0}$ is valid. Suppose that the sequence $\mathcal{S}_{T \rightarrow}^{v_{0}} \mathcal{S}_{T \rightarrow-\rightarrow}^{1} v_{1} \ldots{ }_{-\rightarrow}^{v_{i-1}} \mathcal{S}_{T}^{i}$ is valid. After these topplings, the number of grains on the vertex $v_{i}$ is $\mathcal{S}_{T}^{i}\left(v_{i}\right)=\mathcal{S}_{T}\left(v_{i}\right)+\sum_{j<i} \operatorname{deg}\left(v_{i}, v_{j}\right)$ (recall that $\operatorname{deg}\left(v_{i}, v_{j}\right)$ is the number of edges linking $v_{i}$ and $\left.v_{j}\right)$. We want to prove that $v_{i}$ can be toppled, that is, $\mathcal{S}_{T}^{i}\left(v_{i}\right) \geq \operatorname{deg}\left(v_{i}\right)$. By Lemma 33, any arc $\mathcal{O}_{T}(e)$ is directed toward its least endpoint (for the postfix order) unless $e$ is external $(\mathcal{G}, T)$-active. Let $h$ be an half-edge in an edge linking $v_{i}$ to a vertex $v_{j}, j \geq i$. The vertex $v_{j}$ is less than or equal to $v_{i}$ for the postfix order, hence $h$ is either a tail or an external $(\mathcal{G}, T)$-active half-edge. In both cases, the half-edge $h$ has contribution 1 to $\mathcal{S}_{T}\left(v_{i}\right)$. Hence,

$$
\mathcal{S}_{T}\left(v_{i}\right) \geq \sum_{j \geq i} \operatorname{deg}\left(v_{i}, v_{j}\right)
$$

Thus,

$$
\mathcal{S}_{T}^{i}\left(v_{i}\right)=\mathcal{S}_{T}\left(v_{i}\right)+\sum_{j \geq i} \operatorname{deg}\left(v_{i}, v_{j}\right) \geq \sum_{j \geq 0} \operatorname{deg}\left(v_{i}, v_{j}\right)=\operatorname{deg}\left(v_{i}\right)
$$

and $v_{i}$ can be toppled. By induction, the sequence of topplings $\mathcal{S}_{T}^{v_{0 \rightarrow}} \mathcal{S}_{T \rightarrow-\rightarrow}^{1} v_{1} \ldots$ $\ldots v_{|V|-1} \mathcal{S}_{T}^{|V|}$ is valid.

It remains to prove that $\Lambda: T \mapsto \mathcal{S}_{T}$ is a bijection between the spanning trees and the recurrent sandpile configurations. For this purpose we define a mapping $\Upsilon$ that we shall prove to be the inverse of $\Lambda$. The mapping $\Upsilon$ is a variant of the burning algorithm introduced by Dhar in order to distinguish between recurrent and non-recurrent sandpile configurations [17]. The spanning tree returned by the algorithm can be seen as the path through which the fire (the sequence of topplings) propagates. The intuitive principle of the algorithm is to decompose each toppling and consider its effect grain after grain. When a grain makes another vertex topple, we add the edge by which the grain has traveled into the tree. Different variants of this algorithm have been proposed [10, 8]. These variants differ by the rule used for choosing the next grain to be sent, and also differ from the procedure $\Upsilon$ given below. Let us insist that the variants considered in $[10,8]$ do not contain our bijection $\Lambda$ as a special case.

If $v$ is a vertex and $F \subseteq E$ be a subgraph, we denote by $\operatorname{deg}_{F}(v)$ the degree of $v$ in the subgraph $F$.

Definition 48 Let $\mathcal{G}=\left(H, \sigma, \alpha, h_{0}\right)$ be an embedded graph. The mapping $\Upsilon$ associates with a recurrent sandpile configuration $\mathcal{S}$ the spanning tree defined by the following procedure.

Procedure $\Upsilon$ :

- Initialize the current half-edge $h$ to be $h_{0}^{\prime}=\sigma^{-1}\left(h_{0}\right)$. Initialize the tree $T$ and the set of visited edges $F$ to be empty.

- Core:

C1: Let e be the edge containing $h$, let $u$ be the vertex incident to $h$ and let $v$ be the other 
endpoint of e. If e is not in $F$, then add e to $F$. If in addition $u$ is not connected to $v$ by $T$ and $\mathcal{S}(v)+\operatorname{deg}_{F}(v) \geq \operatorname{deg}(v)$ then add e to $T$.

C2: Move to the next half-edge clockwise around $T$ : if $e$ is in $T$, then set the current half-edge $h$ to be $\sigma^{-1} \alpha(h)$, else set it to be $\sigma^{-1}(h)$.

Repeat steps $\boldsymbol{C 1}$ and $\boldsymbol{C 2}$ until the current half-edge $h$ is $h_{0}^{\prime}$.

- Return the tree T.

We represent the intermediate steps of the procedure $\Upsilon$ in Figure 26.
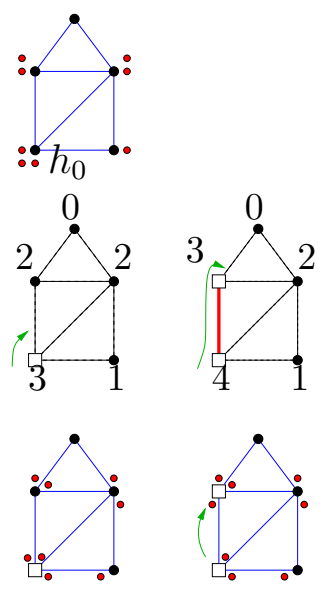
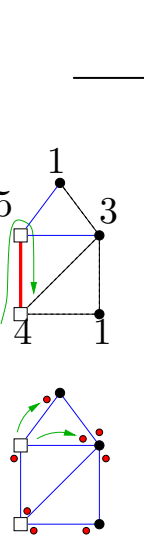

\section{$\Upsilon$}
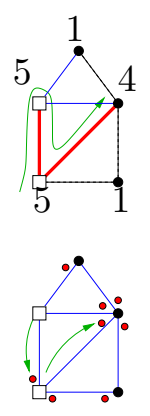
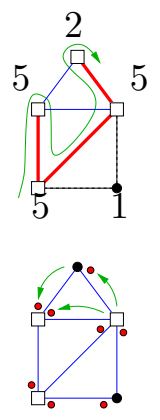
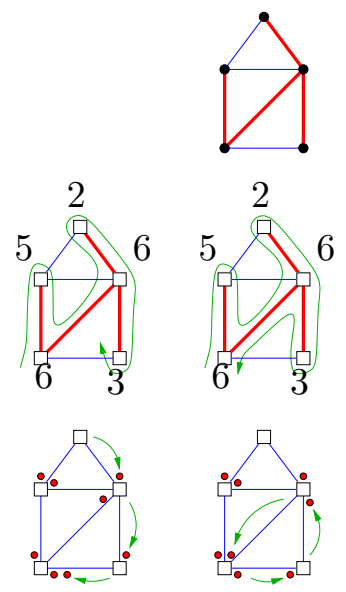

Figure 26: The mapping $\Upsilon$. In the middle line, some intermediate steps are represented. The set $\bar{F}$ of unvisited edges is indicated by dashed lines. The number associated to each vertex $v$ is equal to $\mathcal{S}(v)+\operatorname{deg}_{F}(v)$. In the bottom line, the burning algorithm representation of each of the intermediate steps is given.

Observe that during the procedure $\Upsilon$ our motion (step C2) around the spanning tree is reverse (compared to our previous algorithms). This way of visiting the half-edges would be the usual tour of the spanning tree in the embedded graph $\mathcal{G}^{\prime}=\left(H, \sigma^{-1}, \alpha, h_{0}^{\prime}\right)$.

We will now prove that $\Upsilon$ and $\Lambda$ are inverse bijections. We first prove that the mapping $\Upsilon$ is well defined on recurrent configurations and returns a spanning tree.

Proposition 49 The procedure $\Upsilon$ is well defined on recurrent configurations and returns a spanning tree.

Lemma 50 Let $\mathcal{S}$ be a recurrent configuration. Then, at any time of the execution of the procedure $\Upsilon$ on $\mathcal{S}$, the endpoint $u$ of the current half-edge $h$ is connected to $v_{0}$ by $T$.

Proof. The property holds at the beginning of the execution. Clearly, it remains true each time a step $\mathbf{C} 2$ is performed. 
Proof of Proposition 49. Let $\mathcal{S}$ be a recurrent configuration. We denote by $\Upsilon[\mathcal{S}]$ the execution of the procedure $\Upsilon$ on $\mathcal{S}$. We prove successively the following properties on the execution $\Upsilon[\mathcal{S}]$.

- At any time of the execution, the subgraph $T$ is a tree incident to $v_{0}$. The property holds at the beginning of the execution. Suppose that it holds at the beginning of a given core step and consider the edge $e$ with endpoints $u$ and $v$ containing the current half-edge. If the edge $e$ is added to $T$, the subgraph $T$ remains acyclic since $u$ is not connected to $v$ by $T$. Moreover the subgraph $T$ remains connected and incident to $v_{0}$ since (by Lemma 50 ) the vertex $u$ is connected to $v_{0}$ by $T$.

- No half-edge is visited twice, hence the execution terminates. Suppose that a half-edge $h$ is visited twice during the execution. We consider the first time this situation happens. First note that $h \neq h_{0}^{\prime}$ or the execution would have stopped just before the second visit to $h$. Let $h_{1}$ and $h_{2}$ be respectively the current half-edge just before the first and second visit to $h$.. Let $T_{1}$ and $T_{2}$ be the trees constructed by the procedure $\Upsilon$ at the time of the first and second visit to $h$. Let $e$ be the edge containing $\sigma^{-1}(h)$. For $i=1,2$ we have $h=\sigma^{-1} \alpha\left(h_{i}\right)$ if $e$ is in $T_{i}$ and $h=\sigma^{-1}\left(h_{i}\right)$ otherwise. Since $h_{1} \neq h_{2}$ and $T_{1} \subseteq T_{2}$, the edge $e$ is in $T_{2}$ but not in $T_{1}$. This is impossible since after the visit of $h_{1}$ the edge $e$ is in $F$ and cannot be added to the tree $T$ anymore.

We denote by $T_{0}$ the tree returned by the execution $\Upsilon[\mathcal{S}]$ and by $F_{0}$ the set of visited edges at the end of this execution.

- If $e=\left\{h_{1}, h_{2}\right\}$ is an edge in $T_{0}=\Upsilon(\mathcal{S})$ and the endpoint of $h_{1}$ is the parent of the endpoint of $h_{2}$, then $h_{1}$ is visited during the execution $\Upsilon[\mathcal{S}]$. Consider the core step at which the edge $e$ is added to the tree $T$. Let $h$ be the current half-edge, let $u$ be the vertex incident to $h$ and let $v$ be the other endpoint of $e$. By Lemma 50, the vertex $u$ is connected to $v_{0}$ by $T \subseteq T_{0}-e$, hence $u$ is the parent of $v$. Hence $h_{1}=h$ is visited during the execution $\Upsilon$.

- At the end of the execution, any edge adjacent to $T_{0}$ is in $F_{0}$. We want to show that any half-edge incident to $T_{0}$ is visited during the execution $\Upsilon[\mathcal{S}]$. First observe that no edge can be added to $T$ after its first visit. Therefore, when a step $\mathbf{C} 2$ is performed, the edge $e$ containing the current half-edge is in $T$ if and only if it is in $T_{0}$. Let $h$ be a half-edge incident to $T_{0}$ which has not been visited during the execution $\Upsilon$. If the half-edge $\sigma^{-1}(h)$ is not in $T_{0}$ then it has not been visited (or $h$ would have been the next half-edge visited during the execution). Thus by applying $\sigma^{-1}$ repeatedly we find an unvisited half-edge $h$ such that $\sigma^{-1}(h)$ is in $T_{0}$. Then, the half-edge $\alpha \sigma^{-1}(h)$ has not been visited during the execution $\Upsilon$ (or $h$ would have been the next half-edge visited during the execution). Thus (by the preceding point) the endpoint of $\alpha \sigma^{-1}(h)$ is the child of the endpoint of $\sigma^{-1}(h)$. We have proved that if there is an unvisited half-edge $h$ incident to $T_{0}$, then there is an unvisited half-edge incident to one of its children in $T_{0}$. We reach a contradiction.

- The tree $T_{0}=\Upsilon(\mathcal{S})$ is spanning. Let $v_{0}, v_{1}, \ldots, v_{|V|-1}$ be a labeling of the vertices such that the sequence $\mathcal{S}_{\rightarrow \rightarrow}^{v_{0}} \mathcal{S}^{1}{ }^{v_{1}} \ldots{ }^{v_{|V|-1}} \mathcal{S}^{|V|}$ is valid. In the configuration $\mathcal{S}_{i}$, the number of sand grains on the vertex $v_{i}$ is $\mathcal{S}^{i}\left(v_{i}\right)=\mathcal{S}\left(v_{i}\right)+\sum_{j<i} \operatorname{deg}\left(v_{j}, v_{i}\right)$ and is more than the degree of $v_{i}$. Suppose now that the tree $T_{0}$ is not spanning and consider the least index $i$ such that $v_{i}$ is not connected to $v_{0}$ by $T$. Each vertex $v_{j}$ for $j<i$ is incident to 
$T$, hence (by the preceding point) every edge joining $v_{j}$ and $v_{i}$ is in $F_{0}$. Moreover $v_{i}$ is adjacent to at least one of the vertices $v_{j}, j<i$ since $\mathcal{S}\left(v_{i}\right)$ is less than its degree and $\mathcal{S}^{i}\left(v_{i}\right)$ is not. Consider the last edge $e$ (in order of visit) joining $v_{i}$ to a vertex $v_{j}, j<i$. When the edge $e$ is visited, we have $\operatorname{deg}_{F}\left(v_{i}\right) \geq \sum_{j<i} \operatorname{deg}\left(v_{i}, v_{j}\right)$. Therefore, the condition $\mathcal{S}\left(v_{i}\right)+\operatorname{deg}_{F}\left(v_{i}\right) \geq \operatorname{deg}\left(v_{i}\right)$ holds and the edge $e$ should have been added to the tree $T$. We reach a contradiction.

We proceed to prove that $\Lambda$ and $\Upsilon$ are inverse mappings.

Lemma 51 Consider a given core step of the procedure $\Upsilon$. Let e be the edge containing the current half-edge $h$ and let $v$ be the endpoint of $\alpha(h)$. If the edge $e$ is added to $T$, then the inequality $\mathcal{S}(v)+\operatorname{deg}_{F}(v) \geq \operatorname{deg}(v)$ (tested in the procedure $\Upsilon$ ) is an equality.

Proof. Observe first that the vertex $v$ is distinct from $v_{0}$, otherwise adding $e$ to the tree $T$ would create a cycle by Lemma 50. While $v$ is not connected to $v_{0}$ by $T$, it is not the endpoint of the current half-edge $h$ (Lemma 50). Thus, each time the quantity $\operatorname{deg}_{F}(v)$ increases, that is, each time an edge incident to $v$ is added to $F$, the condition $\mathcal{S}(v)+\operatorname{deg}_{F}(v) \geq \operatorname{deg}(v)$ is tested and the edge is added to $T$ if the condition holds.

Lemma 52 Let $\mathcal{G}=\left(H, \sigma, \alpha, h_{0}\right)$ be an embedded graph and let $T$ be a spanning tree. We consider the $(\mathcal{G}, T)$-order on half-edges. Let $v$ be a vertex distinct from $v_{0}$ and let $h_{v}$ be the half-edge incident to $v$ in the edge of $T$ linking $v$ to its parent. Any half-edge $h$ incident to $v$ and such that $\alpha(h)>h_{v}$ is external. Moreover, there are $\operatorname{deg}(v)-\mathcal{S}_{T}(v)-1$ such half-edges.

Proof. We consider the orientation $\mathcal{O}_{T}$. Recall from Lemma 5 that $\alpha\left(h_{v}\right)<h_{v}$ and that the half-edges $h$ incident to a descendant of $v$ are characterized by $\alpha\left(h_{v}\right)<h \leq h_{v}$. In particular, the inequalities $\alpha\left(h_{v}\right)<h \leq h_{v}$ hold for the half-edges incident to $v$. We now prove successively the following properties.

- Any half-edge $h$ incident to $v$ and such that $\alpha(h)>h_{v}$ is external. Suppose that the half-edge $h$ is internal and consider the edge $e$ containing $h$. If $e$ links $v$ to its parent, then $h=h_{v}$ and $\alpha(h)=\alpha\left(h_{v}\right)<h_{v}$. If $e$ links $v$ to one of its children, then $\alpha(h)$ is incident to a descendant of $v$ and $\alpha(h) \leq h_{v}$. In either cases, the hypothesis $\alpha(h)>h_{v}$ does not hold.

- An external half-edge $h$ incident to $v$ is a non-active head if and only if $\alpha(h)>h_{v}$. The three following properties are sufficient to prove the equivalence:

- If $h$ is a tail then $\alpha(h)<h_{v}$. Indeed, we have $\alpha(h)<h$ since $h$ is a tail and $h \leq h_{v}$ since $h$ is incident to $v$.

- If $h$ is a head and $\alpha(h)<h_{v}$ then $h$ is $(\mathcal{G}, T)$-active. Since $h$ is a head, we have $h<\alpha(h)$ hence, $\alpha\left(h_{v}\right)<h<\alpha(h)<h_{v}$. Thus, $\alpha(h)$ is incident to a descendant of $v$ and the edge $e=\{h, \alpha(h)\}$ is $(\mathcal{G}, T)$-active by Lemma 7 .

- If $h$ is a head and $\alpha(h)>h_{v}$ then $h$ is not $(\mathcal{G}, T)$-active. Since $h$ is a head we have 
$h<\alpha(h)$. Since $\alpha(h)>h_{v}$, the half-edge $\alpha(h)$ is not incident to a descendant of $v$ and the edge $e=\{h, \alpha(h)\}$ is not $(\mathcal{G}, T)$-active by Lemma 7 .

- There are $\operatorname{deg}(v)-\mathcal{S}_{T}(v)-1$ half-edges $h$ incident to $v$ and such that $\alpha(h)>h_{v}$. By definition, $\mathcal{S}_{T}(v)$ is the number of tails plus the number of external $(\mathcal{G}, T)$-active heads incident to $v$. Hence, $\operatorname{deg}(v)-\mathcal{S}_{T}(v)$ is the number of heads incident to $v$ which are not external $(\mathcal{G}, T)$-active. By Lemma 5, internal edges are oriented from parent to child. Hence, the vertex $v$ is incident to exactly one internal head. Thus $\operatorname{deg}(v)-\mathcal{S}_{T}(v)-1$ is the number of external non-active heads. By the preceding point, these half-edges are characterized by the condition $\alpha(h)>h_{v}$.

We now define the clockwise-tour of a tree. Let $\mathcal{G}=\left(H, \sigma, \alpha, h_{0}\right)$ be an embedded graph. Given a spanning tree $T$, we define the clockwise-motion function $\tau$ on half-edges by

$$
\tau(h)=\sigma^{-1} \alpha(h) \text { if } h \text { is internal and } \tau(h)=\sigma^{-1}(h) \text { otherwise. }
$$

As observed above, the clockwise-motion function $\tau$ is the usual motion function for the embedded graph $\mathcal{G}^{-1}=\left(H, \sigma^{-1}, \alpha, \sigma^{-1}\left(h_{0}\right)\right)$. This defines the $\left(\mathcal{G}^{-1}, T\right)$-order on the halfedge set $H$ for which $h_{0}^{\prime}=\sigma^{-1}\left(h_{0}\right)$ is the least element. The $(\mathcal{G}, T)$-order denoted by $<$ and the $\left(\mathcal{G}^{-1}, T\right)$-order denoted by $<^{-1}$ are closely related.

Lemma 53 Let $\mathcal{G}$ be an embedded graph and let $T$ be a spanning tree. The $(\mathcal{G}, T)$-order and $\left(\mathcal{G}^{-1}, T\right)$-order are related by $h<h^{\prime}$ if and only if $\beta\left(h^{\prime}\right)<^{-1} \beta(h)$, where $\beta$ is the involution defined by $\beta(h)=h$ if $h$ is external and $\beta(h)=\alpha(h)$ otherwise.

Proof. Let $t$ be the usual motion function and let $\tau$ be the clockwise-motion function. Observe that $t \beta=\sigma$ and $\tau \beta=\sigma^{-1}$. Thus, $\tau=\beta t^{-1} \beta$. Let us write $t=$ $\left(h_{0}, h_{1}, \ldots, h_{|H|-1}\right)$ in cyclic notation. Then $t^{-1}=\left(h_{|H|-1}, \ldots, h_{1}, h_{0}\right)$ and $\tau=\beta t^{-1} \beta=$ $\left(\beta\left(h_{|H|-1}\right), \ldots, \beta\left(h_{1}\right), \beta\left(h_{0}\right)\right)$. Moreover, $\sigma \beta\left(h_{|H|-1}\right)=t\left(h_{|H|-1}\right)=h_{0}$, hence $\beta\left(h_{|H|-1}\right)=$ $h_{0}^{\prime}=\sigma^{-1}\left(h_{0}\right)$. Therefore, $h_{i}<h_{j}$ if and only if $i<j$ if and only if $\beta\left(h_{j}\right)<^{-1} \beta\left(h_{i}\right)$.

Lemma 54 Let $\mathcal{S}$ be a recurrent configuration and let $T_{0}=\Upsilon(\mathcal{S})$ be the spanning tree returned by the procedure $\Upsilon$. The half-edges of $\mathcal{G}$ are visited in $\left(\mathcal{G}^{-1}, T_{0}\right)$-order during the procedure $\Upsilon$.

Proof. During the procedure $\Upsilon$, no edge can be added to the tree $T$ after its first visit. Therefore, when a step $\mathbf{C 2}$ is applied, the edge $e$ containing the current half-edge is in $T$ if and only if it is in $T_{0}$. Hence, a step C2 corresponds to an application of the clockwisemotion function $\tau$ of the spanning tree $T_{0}$. Since the first visited half-edge is $h_{0}^{\prime}=\sigma^{-1}\left(h_{0}\right)$, the half-edges are visited in $\left(\mathcal{G}^{-1}, T_{0}\right)$-order.

Lemma 55 Let $\mathcal{G}$ be an embedded graph and let $T$ be a spanning tree. Let $v$ be a vertex distinct from $v_{0}$ and let $e_{v}$ be the edge of $T$ linking $v$ to its parent. There are $\operatorname{deg}(v)-$ $\mathcal{S}_{T}(v)-1$ edges incident to $v$ and less than $e_{v}$ for the $\left(\mathcal{G}^{-1}, T\right)$-order. 
Proof. Let $h_{v}$ be the half-edge of $e_{v}$ incident to $v$. Let $h \neq h_{v}$ be a half-edge incident to $v$ and let $e$ be the edge containing $h$. We prove successively the following properties.

- The edge $e$ is less than $e_{v}$ if and only if $\alpha(h)<^{-1} \alpha\left(h_{v}\right)$. Moreover, in this case e is not a loop. By Lemma 5 applied to the embedded graph $\mathcal{G}^{-1}$, the half-edges $h$ incident to $v$ are such that $\alpha\left(h_{v}\right)<^{-1} h \leq^{-1} h_{v}$. Hence, the edge containing $h$ is less than $e_{v}$ for the $\left(\mathcal{G}^{-1}, T\right)$-order if and only if $\alpha(h)<^{-1} \alpha\left(h_{v}\right)$. In this case, $\alpha(h)$ is not incident to $v$ by Lemma 5 , that is, $e$ is not a loop.

- The conditions $\alpha(h)<^{-1} \alpha\left(h_{v}\right)$ and $\alpha(h)>h_{v}$ are equivalent. Moreover, there are $\operatorname{deg}(v)-\mathcal{S}_{T}(v)-1$ half-edges satisfying this condition. Suppose $\alpha(h)<^{-1} \alpha\left(h_{v}\right)$. In this case, $h$ external. Indeed, $h$ is not in $e_{v}$ and is not incident to a child of $v$ by Lemma 5 applied to the embedded graph $\mathcal{G}^{-1}$. Hence, by Lemma 53, we get $\alpha(h)>h_{v}$. Conversely, if $\alpha(h)>h_{v}$, the edge $e$ is external by Lemma 52, hence $\alpha(h)<^{-1} \alpha\left(h_{v}\right)$ by Lemma 53 . By Lemma 52, there are $\operatorname{deg}(v)-\mathcal{S}_{T}(v)-1$ half-edges satisfying this condition.

Proposition 56 The mapping $\Lambda \circ \Upsilon$ is the identity on recurrent configurations.

Proof. Let $\mathcal{S}$ be a recurrent configuration and let $T=\Upsilon(\mathcal{S})$. We want to prove that the recurrent configuration $\mathcal{S}_{T}=\Lambda(T)$ is equal to $\mathcal{S}$. We already know that $\mathcal{S}_{T}\left(v_{0}\right)=\operatorname{deg}\left(v_{0}\right)=\mathcal{S}\left(v_{0}\right)$ since $\mathcal{S}_{T}$ and $\mathcal{S}$ are recurrent configurations. Let $v$ be a vertex distinct from $v_{0}$ and let $e_{v}$ be the edge of $T$ linking $v$ to its parent. Let $F$ be the set of visited edges when $e_{v}$ is added to $T$ during the execution $\Upsilon[\mathcal{S}]$. We know that $\mathcal{S}(v)=\operatorname{deg}(v)-\operatorname{deg}_{F}(v)$ by Lemma 51. It remains to prove that $\mathcal{S}_{T}(v)=\operatorname{deg}(v)-\operatorname{deg}_{F}(v)$. By Lemma 54, the half-edges are visited in $\left(\mathcal{G}^{-1}, T\right)$-order during the execution $\Upsilon[\mathcal{S}]$. Therefore, the value $\operatorname{deg}_{F}(v)$ is the number of edges incident to $v$ which are less or equal to $e_{v}$ for the $\left(\mathcal{G}^{-1}, T\right)$-order. There are $\operatorname{deg}(v)-\mathcal{S}_{T}(v)$ such edges by Lemma 55 . We obtain $\operatorname{deg}_{F}(v)=\operatorname{deg}(v)-\mathcal{S}_{T}(v)$, or equivalently, $\mathcal{S}_{T}(v)=\operatorname{deg}(v)-\operatorname{deg}_{F}(v)$. Thus, $\mathcal{S}_{T}(v)=\mathcal{S}(v)$.

Proposition 57 The mapping $\Upsilon \circ \Lambda$ is the identity on spanning trees.

Proof. Let $T_{0}$ be a spanning tree. We denote by $T_{1}=\Upsilon\left(\mathcal{S}_{T_{0}}\right)$ the image of $T_{0}$ by $\Upsilon \circ \Lambda$ and want to prove that $T_{1}=T_{0}$. Recall that every edge of $\mathcal{G}$ is visited during the execution $\Upsilon\left[\mathcal{S}_{T_{0}}\right]$. Hence, it is sufficient to prove that at the beginning of any core step of the execution $\Upsilon\left[\mathcal{S}_{T_{0}}\right]$, the tree $T$ constructed by the procedure $\Upsilon$ is $T_{0} \cap F$, where $F$ denotes the set of visited edges. We proceed by induction on the number of core steps. The property holds at the beginning of the first core step. Suppose that it holds at the beginning of the $k^{\text {th }}$ core step. If the edge $e$ containing the current half-edge is already in the set $F$ of visited edges, then the set $F$ and the tree $T$ are unchanged during this core step and the property holds at the beginning of the $k+1^{\text {th }}$ core step. Suppose now that the edge $e$ is not in $F$ at the beginning of the $k^{\text {th }}$ core step. By the induction hypothesis, the tree $T$ constructed by the procedure $\Upsilon$ is $T_{0} \cap F$. Moreover, no edge is added to 
the tree $T$ after its first visit, hence $T=T_{1} \cap F$. In other words, the spanning trees $T_{0}$ and $T_{1}$ coincide on $F$. By Lemma 54 , the half-edges are visited in $\left(\mathcal{G}^{-1}, T_{1}\right)$-order during the execution $\Upsilon\left[\mathcal{S}_{T_{0}}\right]$, hence the edges visited before $e$ during the execution $\Upsilon\left[\mathcal{S}_{T_{0}}\right]$ have been visited in $\left(\mathcal{G}^{-1}, T_{0}\right)$-order. Thus, the edges visited before $e$ during the execution $\Upsilon\left[\mathcal{S}_{T_{0}}\right]$ are the edges which are less than $e$ for the $\left(\mathcal{G}^{-1}, T_{0}\right)$-order. Suppose now that the edge $e$ is in the tree $T_{0}$. In this case the endpoints $u$ and $v$ of $e$ are not connected by $T \subseteq T_{0}-e$. Moreover, the value $\operatorname{deg}_{F+e}(v)$ which corresponds to the number of edges incident to $v$ and visited before $e$ during the execution $\Upsilon\left[\mathcal{S}_{T_{0}}\right]$, that is, the edge which are less or equal to $e$ for the $\left(\mathcal{G}^{-1}, T_{0}\right)$-order, is $\operatorname{deg}(v)-\mathcal{S}_{T_{0}}(v)$ by Lemma 55 . Thus, the condition $\mathcal{S}_{T_{0}}(v)+\operatorname{deg}_{F+e}(v) \geq \operatorname{deg}(v)$ (tested by the procedure $\Upsilon$ ) holds and the edge $e$ is added to the tree $T$. Suppose now that $e$ is not in $T_{0}$. In this case, the edge $e_{v}$ linking $v$ to its father in $T_{0}$ is greater than $e$ for the $\left(\mathcal{G}^{-1}, T_{0}\right)$-order. Hence, the value $\operatorname{deg}_{F+e}(v)$ is less or equal to the number of edges incident to $v$ which are less than $e_{v}$ for the $\left(\mathcal{G}^{-1}, T_{0}\right)$-order. Thus, $\operatorname{deg}_{F+e}(v)<\operatorname{deg}(v)-\mathcal{S}_{T_{0}}(v)-1$ by Lemma 55 . The condition $\mathcal{S}_{T_{0}}(v)+\operatorname{deg}_{F+e}(v) \geq \operatorname{deg}(v)$ (tested by the procedure $\Upsilon$ ) does not hold, hence the edge $e$ is not added to the tree $T$. In any case, the property holds at the beginning of the $k+1^{\text {th }}$ core step.

This concludes our proof of Theorem 45.

\section{Concluding remarks}

\subsection{The cycle and cocycle reversing systems}

We consider the cycle reversing system and the cocycle reversing system. A transition in the cycle (resp. cocycle) reversing system consists in flipping a directed cycle (resp. cocycle). The cycle and cocycle reversing systems appear implicitly or explicitly in many works (e.g. $[20,15,24,38,6])$.

It is known from [38] that there is a unique $v_{0}$-connected orientation (equivalently, orientation without head-min directed cocycle by Lemma 27) in each equivalence class of the cocycle reversing system. The counterpart of this property for the cycle reversing system is given by Proposition 34. Indeed, the equivalence classes of the cycle reversing system are in one-to-one correspondence with outdegree sequences [20]. Thus, Proposition 34 proves that there is a unique minimal orientation (that is, orientation without tail-min directed cycle) in each equivalence class of the cycle reversing system.

The cycle-cocycle reversing system in which a transition consists in flipping either a directed cycle or a directed cocycle was introduced in [24]. It was observed in this paper that the cycle and cocycle flips are really independent since they act on the cyclic part and acyclic part respectively and do not modify the other part. As a consequence it was 
shown that the equivalence classes of the cycle-cocycle reversing system are in one-to-one correspondence with root-connected outdegree sequences. Since the cycle and cocycle flips are independent, the uniqueness of the $v_{0}$-connected orientation in the classes of the cocycle reversing system ([38]) and the uniqueness of minimal orientation in the classes of the cycle reversing system (Proposition 34 ) proves that there is a unique $v_{0}$-connected minimal orientation in each equivalence class of the cycle-cocycle reversing system.

As observed in [24], the enumerative results of Theorem 41 can be expressed in terms of cycle/cocycle reversing systems. For instance, the equivalence classes of the cocycle reversing system (in bijection with minimal orientations) are counted by $T_{G}(1,2)$, the equivalence classes of the cocycle reversing system reduced to one element (equivalently, the strongly connected orientations) are counted by $T_{G}(0,2)$ etc.

\subsection{The planar case and duality}

In this subsection we restrict our attention to planar graphs. Our goal is to highlight some nice properties of our bijections with respect to duality. Therefore we will handle simultaneously a planar embedding and its dual. In order to avoid confusion we shall indicate the implicit embedding $\mathcal{G}$ for the tree-intervals and the mapping $\Phi$ by writing $\left[T^{-}, T^{+}\right]_{\mathcal{G}}$ and $\Phi_{\mathcal{G}}$.

Let $G=(V, E)$ be a planar graph. The graph $G$ can be embedded in the sphere, that is, drawn in such a way the edges only intersect at their endpoints. An embedding of $G$ in the oriented sphere defines a combinatorial embedding $\mathcal{G}=(H, \sigma, \alpha)$ where the permutation $\sigma$ corresponds to the counterclockwise order around each vertex. There is a one-to-one correspondence between the embedding of graphs in the oriented sphere and combinatorial embeddings having Euler characteristic 0, where the Euler characteristic is the number of vertices (cycles of $\sigma$ ) plus the number of faces (cycles of $\sigma \alpha$ ) minus the number of edges (cycles of $\alpha$ ) minus 2. We call these embeddings planar. If $\mathcal{G}=\left(H, \sigma, \alpha, h_{0}\right)$ is a (combinatorial) planar embedding, then $\mathcal{G}^{*}=\left(H, \sigma \alpha, \alpha, h_{0}\right)$ correspond to the graphical dual of $\mathcal{G}$ in the reverse-oriented sphere (the graphical dual of a graph embedded in the sphere is obtained by putting a vertex in each face and an edge across each edge). Observe, by the way that $\mathcal{G}^{* *}=\mathcal{G}$.

Consider a planar embedding $\mathcal{G}$. Observe that the edges, subgraphs and orientations of $\mathcal{G}$ can also be considered as edges, subgraphs and orientations of $\mathcal{G}^{*}$. Given a subgraph $S$ of $\mathcal{G}$ we denote by $\bar{S}^{*}$ the co-subgraph, that is, the complement of $S$ considered as a subgraph of $\mathcal{G}^{*}$. Given an orientation $\mathcal{O}$ of $\mathcal{G}$ we denote by $\overline{\mathcal{O}}^{*}$ the co-orientation, that is, the orientation obtained from $\mathcal{O}$ by reversing all arcs considered as an orientation of $\mathcal{G}^{*}$. Observe that for any subgraph $S$ and any orientation $\mathcal{O}$, we have ${\overline{S^{*}}}^{*}=S$ and $\overline{\overline{\mathcal{O}}}^{*}=\mathcal{O}$. From the Jordan Lemma, a subgraph $S$ is connected if and only if the co-subgraph $\bar{S}^{*}$ is acyclic. This implies the well known property (see [37]) that a subgraph $T$ is a spanning tree of $\mathcal{G}$ if and only if the co-subgraph $\bar{T}^{*}$ is a spanning tree of $\mathcal{G}^{*}$. From this property, 
it follows that the fundamental cycle (resp. cocycle) of an internal (resp. external) edge $e$ with respect to $\mathcal{G}$ and $T$ is the fundamental cocycle (resp. cycle) of $e$ with respect to $\mathcal{G}^{*}$ and $\bar{T}^{*}$. Moreover, it follows directly from the definitions that the motion function of the spanning tree $T$ of $\mathcal{G}$ and the motion function of the spanning tree $\bar{T}^{*}$ of $\mathcal{G}^{*}$ are equal. In particular, the $(\mathcal{G}, T)$-order and the $\left(\mathcal{G}^{*}, \bar{T}^{*}\right)$-order are the same. Hence, an edge is $(\mathcal{G}, T)$-active if and only if it is $\left(\mathcal{G}^{*}, \bar{T}^{*}\right)$-active. Thus, the mapping $S \mapsto \bar{S}^{*}$ induces a bijection between the tree-intervals $\left[T^{-}, T^{+}\right]_{\mathcal{G}}$ and $\left[\bar{T}^{*-}, \bar{T}^{*+}\right]_{\mathcal{G}^{*}}$. It follows directly from this property and the definitions that the mappings $\Phi_{\mathcal{G}}$ and $\Phi_{\mathcal{G}^{*}}$ are related by :

$$
\text { for any subgraph } S \text { of } \mathcal{G},{\overline{\Phi_{\mathcal{G}}(S)}}^{*}=\Phi_{\mathcal{G}}\left(\bar{S}^{*}\right) \text {. }
$$

Acknowledgments: This work has benefited from discussions with Éric Fusy, Emeric Gioan, Yvan Le Borgne, Igor Pak, Gilles Schaeffer and Michel Las Vergnas. I would also like to thank the anonymous referee for pointing to me the reference [26]. Last but not least, I would like to thank Mireille Bousquet-Mélou for her very patient reading of early versions of this manuscript.

\section{References}

[1] P. Bak, C. Tang, and K. Wiesenfeld. Self-organized criticality: An explanation of 1/f noise. Phys. Rev. Lett., 59(4):381-384, 1987.

[2] R.A. Bari. Chromatic polynomials and the internal and external activities of Tutte. In A Bondy and eds. USR Murty, editors, Graph Theory and related topics (Academic Press), pages 41-52, 1979.

[3] O. Bernardi. A characterization of the Tutte polynomial via combinatorial embeddings. Ann. Comb., 12(2):139-153, 2008.

[4] A. Björner, I. Lovász, and P.W. Shor. Chip-firing game on graphs. European J. Combin., 12:283-291, 1991.

[5] B. Bollobás. Modern Graph Theory. Springer (Graduate Texts in Mathematics), 1998.

[6] N. Bonichon. A bijection between realizers of maximal plane graphs and pairs of non-crossing Dyck paths. Discrete Math., 298:104-114, 2005.

[7] T. Brylawski and J.G. Oxley. The Tutte polynomial and its applications, chapter 6 in Matroid Applications. Cambridge University Press, 1991.

[8] D. Chebikin and P. Pylyavskyy. A family of bijections between $G$-parking functions and spanning trees. J. Combin. Theory Ser. A, 110:31-41, 2005.

[9] R. Cori. Un code pour les graphes planaires et ses applications. Soc. Math. de France, Astérisque 27, 1975.

[10] R. Cori and Y. Le Borgne. The sand-pile model and Tutte polynomial. Adv. in Appl. Math., 30:44-52, 2003. 
[11] R. Cori and A. Machì. Maps, hypermaps and their automorphisms: a survey. I, II, III. Exposition. Math., 10(5):403-467, 1992.

[12] R. Cori and D. Rossin. On the sandpile group of dual graphs. European J. Combin., 21(4):447-459, 2000.

[13] H.H. Crapo. A higher invariant for matroids. J. Combin. Theory, 2:406-417, 1967.

[14] H.H. Crapo. The Tutte polynomial. Aequationes Math., 3:211-229, 1969.

[15] H. de Fraysseix and P. Ossona de Mendez. On topological aspects of orientations. Discrete Math., 229:57-72, 2001.

[16] P. Ossona de Mendez. Orientations bipolaires. PhD thesis, École des Hautes Études en Sciences Sociales, Paris, 1994.

[17] D. Dhar. Self-organized critical state of sandpile automaton models. Phys. Rev. Lett., 64, 1990.

[18] D. Dhar and S.N. Majumdar. Equivalence between the abelian sandpile model and the $\mathrm{q} \rightarrow 0$ limit of the Potts model. Phys. A, 185:129-145, 1992.

[19] D. Dhar, P. Ruelle, and D.N. Verma. Algebraic aspects of Abelian sandpile model. J. Phys. A, 28:805-831, 1995.

[20] S. Felsner. Lattice structures from planar graphs. Electron. J. Combin., 11(1), 2004.

[21] D.D. Gebhard and B.E. Sagan. Sinks in acyclic orientations of graphs. J. Combin. Theory Ser. B, 80:130-146, 2000.

[22] I.M. Gessel. Enumerative applications of a decomposition for graphs and digraphs. Discrete Math., 139:257-271, 1995.

[23] I.M. Gessel and B.E. Sagan. The Tutte polynomial of a graph, depth-first search, and simplicial complex partitions. Electron. J. Combin., 3(2):R9, 1996.

[24] E. Gioan. Enumerating degree sequences in digraphs and a cycle-cocycle reversing system. European J. Combin., 2007.

[25] E. Gioan and M. Las Vergnas. Activity preserving bijections between spanning trees and orientations in graphs. Discrete Math., 298:169-188, 2005.

[26] G. Gordon and E. McMahon. Interval partitions and activities for the greedoid Tutte polynomial. Adv. in Appl. Math., 18:33-49, 1997.

[27] G. Gordon and L. Traldi. Generalized activities and the Tutte polynomial. Discrete Math., 85:167-176, 1990.

[28] C. Greene and T. Zaslavsky. On the interpretation of Whitney numbers through arrangements of hyperplanes, zonotopes, non-Radon partitions, and orientations of graphs. Trans. Amer. Math. Soc., 280:97-126, 1983.

[29] D.J. Kleitman and K.J. Winston. Forests and score vectors. Combinatorica, 1:49-54, 1981.

[30] D. Kostic and C.H. Yan. Multiparking functions, graph searching and the tutte polynomial. ArXiv: math.CO/0607602, 2006. 
[31] M. Las Vergnas. Matroïdes orientables. C. R. Acad. Sci. Paris Sér. A-B, 280, 1975.

[32] M. Las Vergnas. The Tutte polynomial of a morphism of matroids. II. Activities of orientations. Progress in Graph Theory (Waterloo, Ont.), pages 367-380, 1984.

[33] B. Lass. Orientations acycliques et le polynôme chromatique. Europ. J. Combinatorics, 22:1001-1023, 2001.

[34] C. Merino. Chip firing game and the Tutte polynomial. Ann. Combin., 3:253-259, 1997.

[35] G.J. Minty. On the axiomatic foundations of the theories of directed linear graphs, electrical networks and network programming. Indiana Univ. Math. J., 15:485-520, 1966.

[36] B. Mohar and C. Thomassen. Graphs on surfaces. J. Hopkins Univ. Press, 2001.

[37] R.C. Mullin. On the enumeration of tree-rooted maps. Canad. J. Math., 19:174-183, 1967.

[38] J. Propp. Lattice structure for orientations of graphs. Manuscript: http://faculty.uml.edu/jpropp/orient.html, 1993.

[39] B. Shapiro and A. Postnikov. Trees, parking functions, syzygies, and deformation of monomial ideals. Trans. Amer. Math. Soc., 356(8):3109-3142, 2004.

[40] R.P. Stanley. Acyclic orientations of graphs. Discrete Math., 5:171-178, 1973.

[41] R.P. Stanley. Decomposition of rational polytopes. Ann. Discrete Math., 6:333-342, 1980.

[42] W.T. Tutte. A ring in graph theory. Proc. Cambridge Philos. Soc., 43:26-40, 1947.

[43] W.T. Tutte. A contribution to the theory of chromatic polynomials. Canad. J. Math., 6:80-91, 1954.

[44] H. Whitney. A logical expansion in mathematics. Bull. Amer. Math. Soc., 38:572$579,1932$.

[45] R.O. Winder. Partitions of $N$-space by hyperplanes. SIAM J. Appl. Math., 14:811818, 1966.

[46] T. Zaslavsky. Facing up to arrangements: face-count formulas for partitions of space by hyperplanes. Mem. Amer. Math. Soc., 1975. 